Article

\title{
Novel Substituted Purine Isosteres: Synthesis, Structure-Activity Relationships and Cytotoxic Activity Evaluation
}

\author{
Spyridon Dimitrakis ${ }^{1}$, Efthymios-Spyridon Gavriil ${ }^{1,+}{ }^{+}$, Athanasios Pousias ${ }^{1}$, Nikolaos Lougiakis ${ }^{1}$ (D, \\ Panagiotis Marakos ${ }^{1, *}$, Nicole Pouli ${ }^{1}$, Katerina Gioti ${ }^{2}$ and Roxane Tenta ${ }^{2}$ (D)
}

1 Department of Pharmacy, Division of Pharmaceutical Chemistry, National and Kapodistrian University of Athens, Panepistimiopolis, 15771 Zografou, Greece; sdimitrakis@pharm.uoa.gr (S.D.); efthymios-spyridon.gavriil@cruk.manchester.ac.uk (E.-S.G.); thanpous@pharm.uoa.gr (A.P.); nlougiak@pharm.uoa.gr (N.L.); pouli@pharm.uoa.gr (N.P.)

2 Department of Nutrition \& Dietetics, School of Health Sciences and Education, Harokopio University, 17671 Athens, Greece; catherine_geo@yahoo.com (K.G.); rtenta@hua.gr (R.T.)

* Correspondence: marakos@pharm.uoa.gr

† Current address: Drug Discovery Unit, Cancer Research UK Manchester Institute, The University of Manchester, Alderley Park SK10 4TG, UK.

check for updates

Citation: Dimitrakis, S.; Gavriil, E.-S.; Pousias, A.; Lougiakis, N.; Marakos, P.; Pouli, N.; Gioti, K.; Tenta, R. Novel Substituted Purine Isosteres: Synthesis, Structure-Activity Relationships and Cytotoxic Activity Evaluation. Molecules 2022, 27, 247. https://doi.org/10.3390/ molecules 27010247

Academic Editor: Naresh Kumar

Received: 25 November 2021

Accepted: 30 December 2021

Published: 31 December 2021

Publisher's Note: MDPI stays neutral with regard to jurisdictional claims in published maps and institutional affiliations.

Copyright: (C) 2021 by the authors. Licensee MDPI, Basel, Switzerland. This article is an open access article distributed under the terms and conditions of the Creative Commons Attribution (CC BY) license (https:// creativecommons.org/licenses/by/ $4.0 /)$.

\begin{abstract}
A number of pyrrolo[2,3-c]pyridines, pyrrolo[3,2- $d$ ]pyrimidines and pyrazolo[4,3- $d$ ]pyrimidines were designed and synthesized as antiproliferative agents. The target compounds possessed selected substituents in analogous positions on the central scaffold that allowed the extraction of interesting SARs. The cytotoxic activity of the new derivatives was evaluated against prostatic (PC-3) and colon (HCT116) cell lines, and the most potent analogues showed $\mathrm{IC}_{50}$ values in the $\mathrm{nM}$ to low $\mu \mathrm{M}$ range, while they were found to be non-toxic against normal human fibroblasts (WI-38). Flow cytometric analysis of DNA content revealed that the most promising derivative $14 \mathrm{~b}$ caused a statistically significant accumulation of PC-3 cells at $\mathrm{G}_{2} / \mathrm{M}$ phase and induced apoptosis in PC-3 cells.
\end{abstract}

Keywords: purine isosteres; pyrrolopyridine; pyrrolopyrimidine; pyrazolopyrimidine; synthesis; cytotoxic activity; cell cycle perturbation; apoptosis

\section{Introduction}

Azaindole and related nitrogen-containing fused heterocycles, presenting structural analogy to adenine base of DNA, are important building blocks of bioactive compounds, and they have, therefore, been extensively investigated in the field of medicinal chemistry [1-3]. Among this structurally diverse class, many pyrazolopyridine and pyrrolopyrimidine derivatives were found to display potent in vitro and in vivo activity, as receptor modifiers or enzyme inhibitors [4,5], possessing interesting antiproliferative [6-8], antibacterial [9,10], antiviral [11,12], antimalarial [13], antileishmanial [14], antidiabetic [15], trypanocidal [16] and neuroprotective properties [17]. The exact heterocyclic isomer, depending on the structural arrangement of nitrogen atoms in the central purine-like ring system, along with the introduction of selected substituents, is important for the interaction of each compound with specific cellular substrates. The classical bioisosteric replacement of a $\mathrm{CH}$ group with a $\mathrm{N}$ atom (vide infra) in heteroaromatic rings can potentially affect a number of the physicochemical parameters of the central scaffold, as well as its intraand intermolecular orbital, steric, electrostatic and hydrophobic interactions, which can translate into improved pharmacological profiles. This minor structural modification is usually challenging from a synthetic point of view, but it provides an excellent opportunity for lead optimization. [18] Although the antitumor activity of these compounds can be attributed to a broad range of molecular targets and several mechanisms, the more interesting targets and mechanisms are certainly related to the inhibition of receptor or 
cytoplasmic protein kinases, which are linked to signal transduction networks associated with carcinogenesis, progression and metastasis of a variety of aggressive human cancers [19]. Purine isosteres usually compete successfully with ATP for interaction with the hinge region of the binding pocket of these enzymes and, therefore, are estimated to be non-selective kinase inhibitors. However, a reasonable degree of selectivity can be achieved as a consequence of the introduction of a variety of substituents, mainly on the respective 2-, 6-and 9-positions of the purine nucleus. For example, the pyrrolopyridine derivative vemurafenib is a mutant specific kinase inhibitor currently used for treatment of metastatic BRAFV600E melanoma [20]. Extensive lead optimization studies are essential in each case to enhance interactions with binding pockets, neighboring the hinge region, in order to improve potency and target selectivity. Nevertheless, the great number of protein kinases, the complexity of cellular signal transduction pathways and the possibility of interactions with additional cellular substrates mean that experimental studies aimed at the clarification of the precise pharmacological function of individual derivatives are challenging.

On the other hand, there is an increasing need for the development of novel, safer and tolerable chemotherapeutic agents for more effective cancer treatment. The identification and targeting of the molecular drivers of cancer remain a major scientific subject and contribute towards the discovery of new compounds, able to inhibit uncontrolled cancer cell proliferation, with low toxicity to normal cells and tissues. These targeted therapies are considered a promising approach for the treatment of human malignancies and have already been proven effective in the clinic, providing impressive-though transitory-responses to patients with advanced cancers. They are well-tolerated drugs, characterized by few off-target effects, low toxicity profiles and fairly broad therapeutic windows. However, the development of therapeutic strategies to improve the long-term effectiveness and limit the emerging resistance in targeted therapies remain a vital challenge in this field. [21].

The starting point for the present study was an attractive pyrazolopyridine hit, namely 3-phenyl-7-(3,4,5-trimethoxyphenyl)aminopyrazolo[3,4-c]pyridine, previously identified by our group [22]. This compound was endowed with strong cytotoxic properties against a variety of cancer cell lines, with $\mathrm{IC}_{50}$ values in the medium to high $\mathrm{nM}$ range. In the course of our involvement in the discovery of new purine analogues with antiproliferative activity [23-25], we considered it interesting to modify the main scaffold of the above-mentioned derivative and insert suitable substituents in order to gain insight into the SARs of this class of compounds. More precisely, we have replaced the pyrazolopyridine central scaffold by three purine-like heterocycles, namely pyrrolo[2,3-c]pyridine, pyrrolo[3,2- $d]$ pyrimidine and pyrazolo[4,3-d]pyrimidine. Besides the 7-(3,4,5-trimethoxyphenyl)- substituent of the lead compound, we have also introduced a phenylamino- and a 4-methylpiperazin-1-yl substituent in the corresponding position of the above-mentioned scaffolds. We preserved the lead compound's 3-phenyl group, and we also prepared the corresponding 3-(3-fluorophenyl)- analogues. We, thus, present herein the synthesis of these series of derivatives and the evaluation of their biological activity.

\section{Results and Discussion}

\subsection{Chemistry}

For the preparation of the series of pyrrolopyridines, we used the intermediate chlorides $\mathbf{9 a}, \mathbf{b}$, which were synthesized as depicted in Scheme 1. 2-Aminopicoline (1) was successively nitrated, diazotized and chlorinated to provide the nitropyridine 4 [26]. The reaction of 4 with DMF-dimethylacetal provided the enamine 5 that upon reduction was cyclized to result in the 7 -chloropyrrolopyridine 6 [27] in $25 \%$ overall yield. The latter was iodinated, the pyrrole nitrogen was protected upon treatment with 4-methoxybenzylchloride (PMB-Cl) and the resulting iodide 8 underwent a Suzuki-type coupling with suitable boronic acids to give the chlorides $\mathbf{9 a}, \mathbf{b}$. All these reactions proceeded in high yields, and compounds were rationally characterized via ${ }^{1} \mathrm{H}-\mathrm{NMR}$ data. It is of interest to notice the characteristic chemical shift of the iodine bearing carbon (C-3) of compounds 7 and 8, which resonated at $56 \mathrm{ppm}$ in the corresponding ${ }^{13} \mathrm{C}-\mathrm{NMR}$ spectra. The chlo- 
rides $9 \mathbf{a}, \mathbf{b}$ were then treated with aniline, or 3,4,5-trimethoxyaniline in the presence of potassium tert-butoxide, tris(dibenzylideneacetone)dipalladium(0) $\left[\mathrm{Pd}_{2}(\mathrm{dba})_{3}\right]$ and $2,2^{\prime}-$ bis(diphenylphosphino)-1,1'-dinapthalene (BINAP), to result in the amines 10a-d in good yields (60-91\%). In parallel, since the nucleophilic substitution of the 7-chloro group of compounds 9 by alkylamines upon heating in DMF or DMSO was not successful, $9 \mathbf{a}, \mathbf{b}$ were treated with m-chloroperoxybenzoic acid and were converted in good yield (88\%) to the corresponding $N$-oxides $\mathbf{1 1 a}, \mathbf{b}$. These derivatives underwent nucleophilic substitution by $\mathrm{N}$-methylpiperazine to provide the aminosubstituted compounds $\mathbf{1 2} \mathbf{a}, \mathbf{b}$ in moderate yield $(50 \%)$, which upon reduction gave the intermediates $\mathbf{1 3} \mathbf{a}, \mathbf{b}$. An interesting and indicative feature in the ${ }^{13} \mathrm{C}$-NMR spectral data of the $N$-oxides $\mathbf{1 1} \mathbf{a}, \mathbf{b}$ and $\mathbf{1 2} \mathbf{a}, \mathbf{b}$ was the chemical shift of C-5, which appears upfield, at 133-134 ppm, when compared to the corresponding peak of compounds $\mathbf{9 a , b}$ and $\mathbf{1 3 a} \mathbf{b} \mathbf{b}$ (C-5 resonance in this case is $137-138 \mathrm{ppm}$ ); this was obviously due to the presence of the neighboring $N$-oxide. Finally, the N1-substituted derivatives $\mathbf{1 0} \mathbf{a}-\mathbf{d}$ and $\mathbf{1 3} \mathbf{a}, \mathbf{b}$ were efficiently deprotected to result in the target pyrrolopyridines $\mathbf{1 4 a - f}$ in good to quantitative yields. In the ${ }^{1} \mathrm{H}-\mathrm{NMR}$ spectra of compounds $14 \mathbf{a}-\mathbf{f}$, the pyrrole $\mathrm{H}$ was detectable as a broad singlet at 10.5-11.8 ppm, whereas the $\mathrm{NH}$ of the 7-substituent of 14a-d was detected upfield at 8.6-9.4 ppm.

The pyrrolopyrimidine analogues were prepared according to the reaction sequence presented in Scheme 2 from isoxazole (15), which-following a previously reported procedure-was converted to the pyrrolecarboxylate 18 [28]. The latter was, in turn, ringclosed; the resulting pyrimidinone 19 [29] was converted to the chloride 20 [30], which was successively iodinated to give $\mathbf{2 1}$ [31]. The iodide 21 was then protected to provide almost quantitatively 22, which was introduced to a Suzuki-type coupling, resulting in high yields of the 3-arylsubstituted chlorides $\mathbf{2 3} \mathbf{a}, \mathbf{b}$. The above-mentioned chlorides were used for the synthesis of the target pyrrolopyrimidines $\mathbf{2 6 a - f}$, obtained in very good overall yields, following an analogous procedure to that reported for the corresponding pyrrolopyridines. A common pattern was obvious at the ${ }^{1} \mathrm{H}-\mathrm{NMR}$ spectra of $\mathbf{2 6 a}-\mathbf{f}$, concerning the pyrrole $\mathrm{NH}$ that appeared as a broad singlet at 10.7-11.1 ppm and the NH of the 4-substituent of compounds $\mathbf{2 6 a}-\mathbf{d}(8.5-8.7 \mathrm{ppm})$, as well as the characteristic chemical shifts of H-2 (8.4-8.5 ppm) and H-6 (7.9-8.1 ppm).

Finally, the pyrazolopyrimidine analogues were synthesized, as shown in Scheme 3, starting from the acetophenones 27 (commercially available) or 29 [32]. The acetophenones were subjected to side chain elongation upon reaction with ethyl oxalate, and the resulting dioxobutyrates 30a [33] and 30b [34] were converted in good yields (85\%) to the corresponding $\alpha$-(hydroxyimino)benzopyruvates 31a [35] and 31b, respectively. Compounds $\mathbf{3 1} \mathbf{a}, \mathbf{b}$ were treated with hydrazine hydrate and were ring-closed with the concomitant reduction of the oxime group to result in the aminopyrazole-carboxylates 32a [36] and $\mathbf{3 2} \mathbf{b}$, in moderate yields $(40 \%)$. The reaction of the carboxylates $\mathbf{3 2} \mathbf{a}, \mathbf{b}$ with formamidine gave the pyrazolopyrimidinones 33a [37] and 33b, which-through the corresponding chlorides $\mathbf{3 4} \mathbf{a}, \mathbf{b}$ and the displacement of the chlorine by suitable amines-resulted in the target pyrazolopyrimidines 35a-f in good overall yields (over $40 \%$ for three steps). All new compounds were unambiguously identified using NMR and mass spectrometry data. 


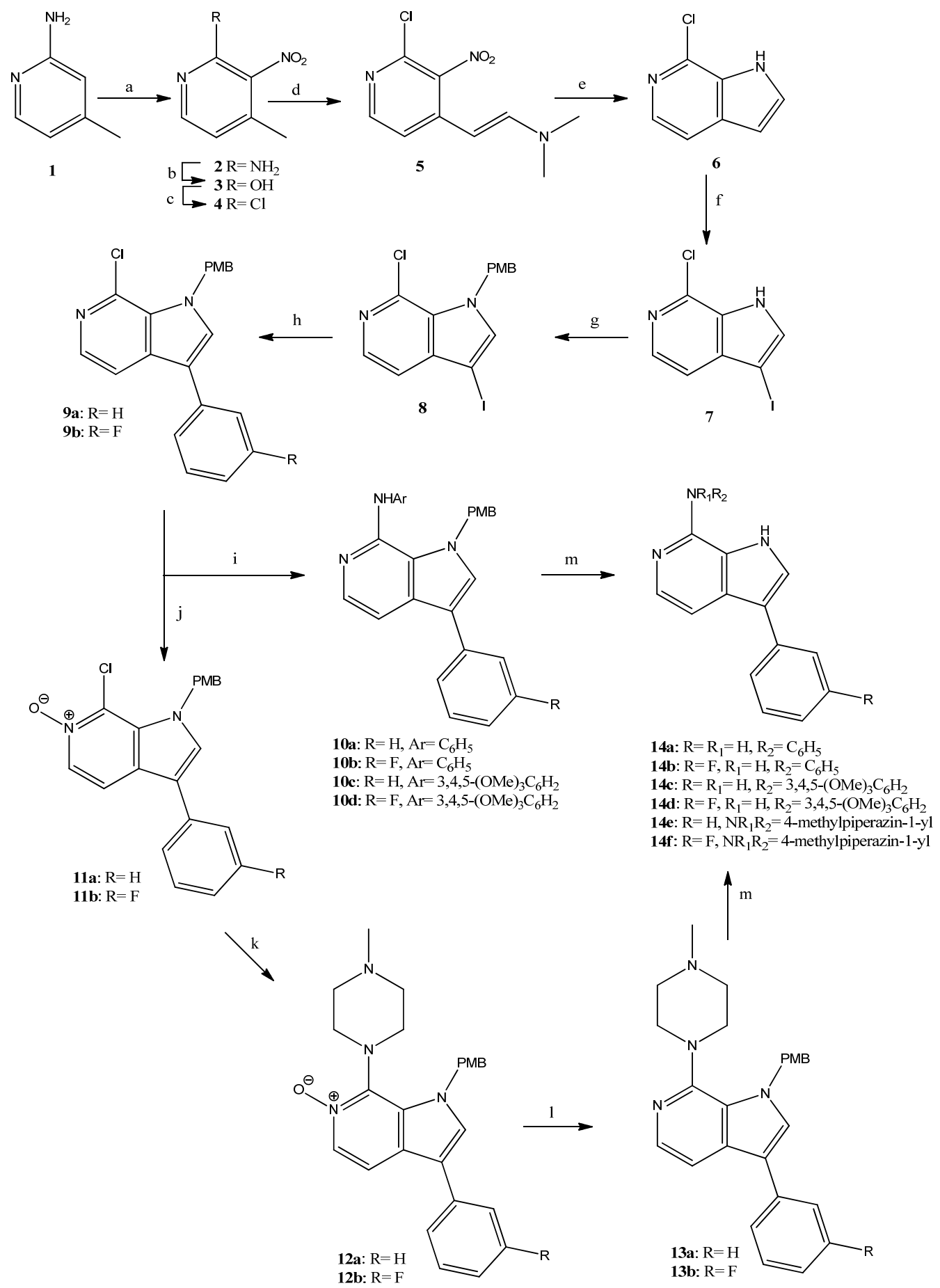

Scheme 1. Reagents and conditions. a: $\mathrm{HNO}_{3}, \mathrm{H}_{2} \mathrm{SO}_{4}, 60{ }^{\circ} \mathrm{C}, 4 \mathrm{~h}, 44 \%$; b: $\mathrm{NaNO}_{2}, \mathrm{H}_{2} \mathrm{SO}_{4}, \mathrm{rt}, 2 \mathrm{~h}, 90 \%$; c: $\mathrm{POCl}_{3}$, reflux, 4 h, 94\%; d: DMF-DMA, DMF, $120^{\circ} \mathrm{C}, 2 \mathrm{~h}, 80 \%$; e: Fe, AcOH, AcONa, EtOH/ $\mathrm{H}_{2} \mathrm{O}$, reflux, 2 h, 82\%; f: NIS, MeOH, rt, 1 h, 99\%; g: NaH, 4-methoxybenzylchloride (PMB-Cl), DMF, rt, 90 min, 99\%; h: phenylboronic acid or 3-fluorophenylboronic acid, $\mathrm{K}_{2} \mathrm{CO}_{3}$, tetrakis(triphenylphosphine)palladium, toluene/EtOH, reflux, $3 \mathrm{~h}, 88-98 \%$; $\mathrm{i}$ : aniline or 3,4,5-trimethoxyaniline, $\mathrm{Pd}_{2}\left(\mathrm{dba}_{3}, \mathrm{BINAP}, t\right.$ BuOK, toluene, reflux, 3.5 h, 60-91\%; j: m-chloroperoxybenzoic acid, $\mathrm{CH}_{2} \mathrm{Cl}_{2}$, rt, 72 h, 88\%; k: 1methylpiperazine, $\mathrm{EtOH}$, autoclave, $120^{\circ} \mathrm{C}, 24 \mathrm{~h}, 50 \%$; : $\mathrm{PCl}_{3}, \mathrm{CHCl}_{3}, \mathrm{rt}, 20 \mathrm{~h}, 72-99 \% ; \mathrm{m}: \mathrm{CF}_{3} \mathrm{CO}_{2} \mathrm{H}$, $60{ }^{\circ} \mathrm{C}, 20 \mathrm{~h}, 91-99 \%$. 

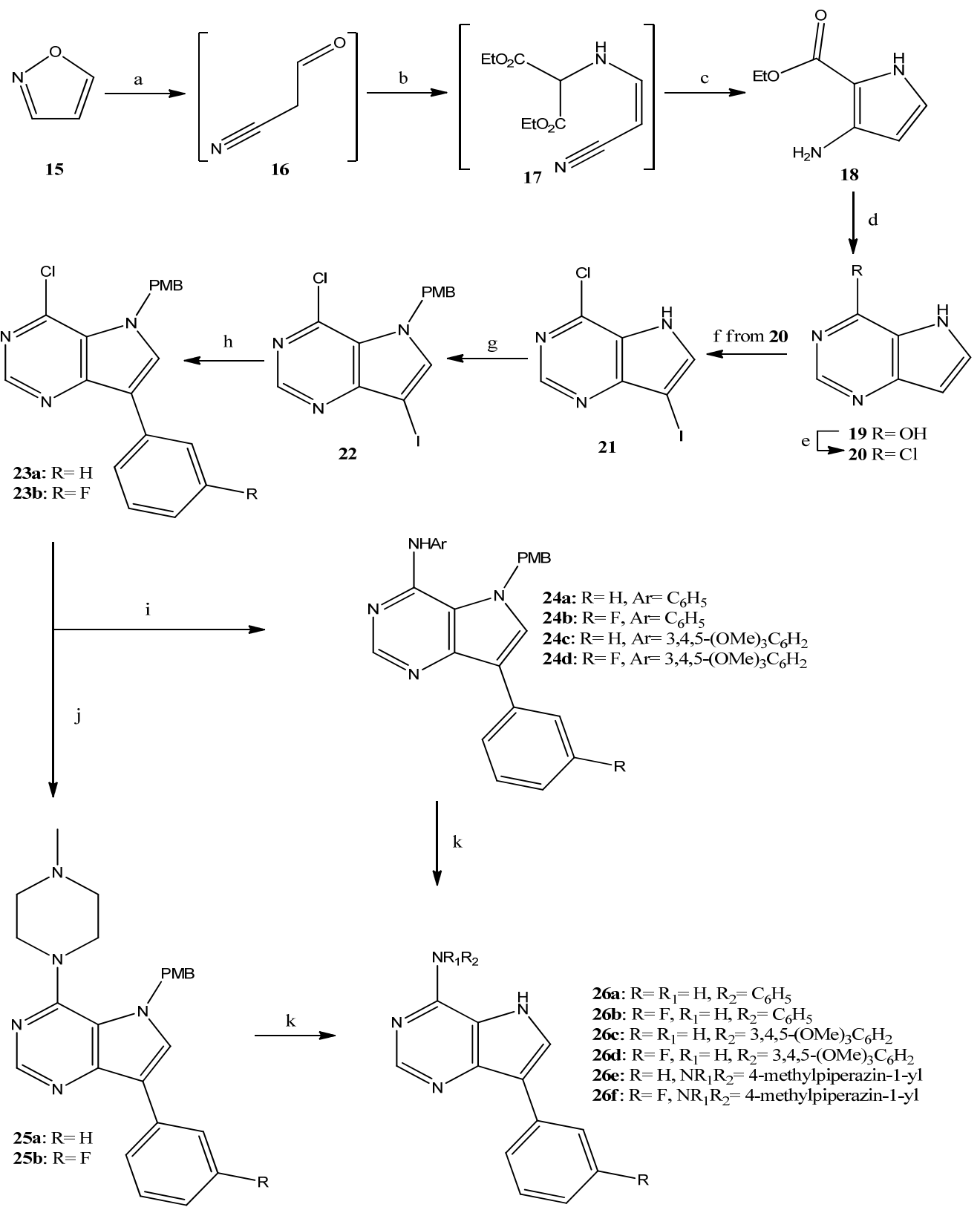

Scheme 2. Reagents and conditions. a: EtONa, EtOH, rt, $30 \mathrm{~min}$; b: ethyl aminomalonate hydrochloride, $\mathrm{AcOH}, \mathrm{AcONa}, \mathrm{rt}, 48$ h; c: EtONa, EtOH, rt, 5 days, 37\% for 3 steps; d: formamidine acetate, EtOH, reflux, 20 h, 82\%; e: $\mathrm{POCl}_{3}$, reflux, 90 min, 88\%; f: NIS, $\mathrm{MeOH}, \mathrm{rt}, 1 \mathrm{~h}, 76 \%$; g: $\mathrm{NaH}, 4-$ methoxybenzylchloride (PMB-Cl), DMF, rt, $90 \mathrm{~min}, 99 \%$; $\mathrm{h}$ : phenylboronic acid or 3-fluorophenylboronic acid, $\mathrm{K}_{2} \mathrm{CO}_{3}$, tetrakis(triphenylphosphine)palladium, toluene/EtOH, reflux, $3 \mathrm{~h}, 90-94 \%$; i: aniline or 3,4,5-trimethoxyaniline, $\mathrm{Pd}_{2}(\mathrm{dba})_{3}$, BINAP, $t$-BuOK, toluene, reflux, $5 \mathrm{~h}, 60-87 \%$; j: 1-methylpiperazine, DMSO, $120{ }^{\circ} \mathrm{C}, 20 \mathrm{~h}, 97-99 \% ; \mathrm{k}: \mathrm{CF}_{3} \mathrm{CO}_{2} \mathrm{H}, 60{ }^{\circ} \mathrm{C}, 20 \mathrm{~h}, 91-99 \%$. 

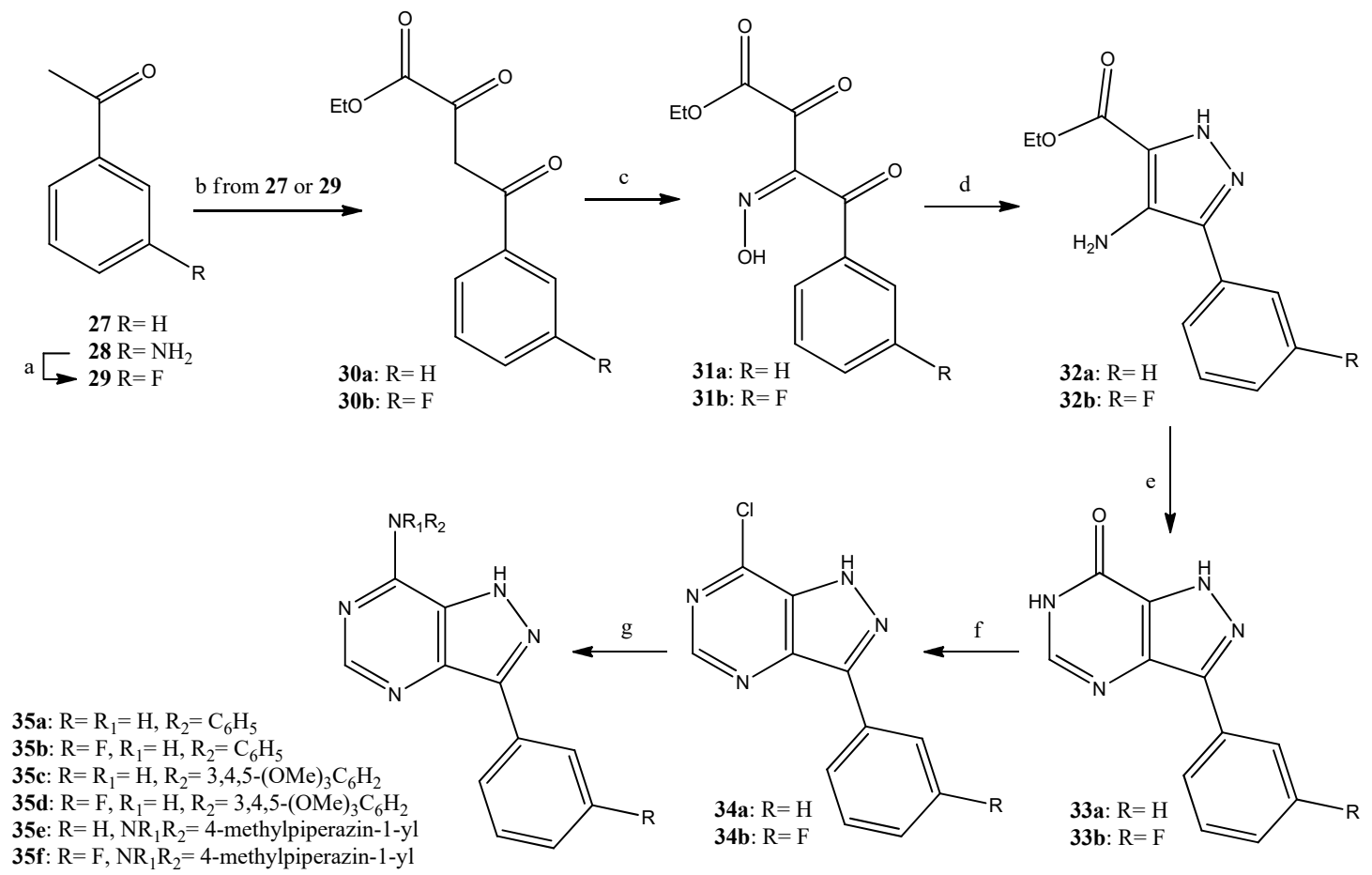

Scheme 3. Reagents and conditions. a: $\mathrm{BF}_{3} \cdot\left(\mathrm{Et}_{2} \mathrm{O}\right)_{2}$, isoamyl nitrite, 1,2-dichlorobenzene, $90{ }^{\circ} \mathrm{C}, 45$ min, $54 \%$; b: $\mathrm{NaH}$, diethyl oxalate, toluene, $50{ }^{\circ} \mathrm{C}, 1 \mathrm{~h}, 99 \%$; $: \mathrm{NaNO}_{2}, \mathrm{AcOH}, 10{ }^{\circ} \mathrm{C}, 1 \mathrm{~h}, 85 \%$; : hydrazine hydrate, $\mathrm{EtOH}, 45^{\circ} \mathrm{C}, 2 \mathrm{~h}, 40 \%$; e: formamidine acetate, $\mathrm{BuOH}$, reflux, $20 \mathrm{~h}, 61 \%$; f: $\mathrm{POCl}_{3}$, $\mathrm{PCl}_{5}$, reflux, $3 \mathrm{~h}, 80 \%$; g: aniline or 3,4,5-trimethoxyaniline or 1-methylpiperazine, EtOH, reflux, $2 \mathrm{~h}$, $57-80 \%$.

\subsection{Biological Evaluation}

The cytotoxic activity of the new compounds was tested against two cancer cell lines, prostatic (PC-3) and colon (HCT116) cell lines (Table 1), while the most potent derivatives were further tested against normal human fibroblasts (WI-38) (Table 2).

Concerning the $N$-(p-methoxybenzyl) substituted pyrrolopyridines $\mathbf{1 0 a}-\mathbf{d}$ and $\mathbf{1 3} \mathbf{a}, \mathbf{b}$, only a few of them possessed moderate antiproliferative activity, namely the 7-phenylamino derivative 10a, together with both the 7-(N-methylpiperazine) substituted derivatives 13a,b, with $\mathrm{IC}_{50}$ values in the range of $2.4-9.3 \mu \mathrm{M}$. On the other hand, the deprotection of the above-mentioned compounds provided derivatives $14 a-f$, which were endowed with very potent cytotoxic properties, with $\mathrm{IC}_{50}$ values in the $\mathrm{nM}$ to low $\mu \mathrm{M}$ range against both cell lines tested. The most potent compound of this group is the 3-(3-fluorophenyl) analogue $\mathbf{1 4 b}$, possessing $\mathrm{IC}_{50}$ values of $55 \mathrm{nM}$ and $90 \mathrm{nM}$ against the prostate and colon cell lines, respectively.

In the pyrrolopyrimidine series, the $N$-(p-methoxybenzyl) substituted analogues $44 a-$ $\mathbf{d}$ and $\mathbf{2 5 a}, \mathbf{b}$ are, in analogy to the previous series, devoid of activity, with the sole exception of 24c, which appeared to be cytotoxic against PC-3 cells $\left(\mathrm{IC}_{50}: 2.25 \mu \mathrm{M}\right)$. Concerning the corresponding deprotected analogues, the 7-(3-fluorophenyl)-4-phenylamino derivative $\mathbf{2 6 b}$ is the most potent compound of this group, followed by the corresponding 7-phenylsubstituted compound 26a. The 4-(3,4,5-trimethoxyphenyl)amino-substituted derivatives $\mathbf{2 6 c}$, d had moderate activity, while a complete loss of activity was observed in the corresponding 4-(N-methylpiperazine)-substituted analogues.

Finally, in the case of pyrazolopyrimidines, the 3-(3-fluorophenyl)-7-phenylamino derivative $35 \mathrm{~b}$ possessed very interesting cytotoxicity, whereas among the remaining compounds, only 35a and 35c showed moderate cytotoxicity, and again the $N$-methylpiperazine substituted analogues were inactive. 
Physicochemical characteristics of the thirty compounds tested were calculated using SwissADME platform [38] and are summarized in Table S1. They were further entered as inputs along with the $\mathrm{IC}_{50} \mathrm{~s}$ in the SIMCA software [39]. A multivariate analysis showed that there is a very weak correlation $(\mathrm{R} 2<0.25)$ between activity and molar refractivity, while there was no correlation between activity and $\log P$.

Table 1. Accumulative results of the antiproliferative activities of all compounds tested.

\begin{tabular}{|c|c|c|c|c|c|c|c|}
\hline \multirow[t]{2}{*}{ Compd } & \multirow[b]{2}{*}{$X$} & \multirow[b]{2}{*}{$\mathbf{Y}$} & \multirow[b]{2}{*}{$\mathbf{R}_{1}{ }^{b}$} & \multirow[b]{2}{*}{$\mathbf{R}_{2}$} & & \multicolumn{2}{|c|}{ IC $_{50}$ Values $(\mu \mathrm{M})^{a}$} \\
\hline & & & & & $\mathbf{R}_{3}{ }^{c}$ & PC-3 & HCT116 \\
\hline $10 a$ & $\mathrm{CH}$ & $\mathrm{CH}$ & PMB & $\mathrm{H}$ & $\mathrm{PhNH}$ & $>10$ & $2.4 \pm 0.1$ \\
\hline $10 \mathrm{~b}$ & $\mathrm{CH}$ & $\mathrm{CH}$ & PMB & $\mathrm{F}$ & $\mathrm{PhNH}$ & $>10$ & $>10$ \\
\hline $10 c$ & $\mathrm{CH}$ & $\mathrm{CH}$ & PMB & $\mathrm{H}$ & TMPNH & $9.80 \pm 0.25$ & $9.40 \pm 0.53$ \\
\hline $10 \mathrm{~d}$ & $\mathrm{CH}$ & $\mathrm{CH}$ & PMB & $\mathrm{F}$ & TMPNH & $>10$ & $>10$ \\
\hline $13 a$ & $\mathrm{CH}$ & $\mathrm{CH}$ & PMB & $\mathrm{H}$ & PIP & $7.00 \pm 0.40$ & $5.00 \pm 0.18$ \\
\hline $13 b$ & $\mathrm{CH}$ & $\mathrm{CH}$ & PMB & $\mathrm{F}$ & PIP & $9.30 \pm 0.76$ & $4.40 \pm 0.35$ \\
\hline $14 a$ & $\mathrm{CH}$ & $\mathrm{CH}$ & $\mathrm{H}$ & $\mathrm{H}$ & $\mathrm{PhNH}$ & $0.215 \pm 0.025$ & $5.00 \pm 0.10$ \\
\hline $14 b$ & $\mathrm{CH}$ & $\mathrm{CH}$ & $\mathrm{H}$ & $\mathrm{F}$ & $\mathrm{PhNH}$ & $0.055 \pm 0.015$ & $0.09 \pm 0.01$ \\
\hline $14 c$ & $\mathrm{CH}$ & $\mathrm{CH}$ & $\mathrm{H}$ & $\mathrm{H}$ & TMPNH & $0.47 \pm 0.12$ & $0.72 \pm 0.09$ \\
\hline $14 d$ & $\mathrm{CH}$ & $\mathrm{CH}$ & $\mathrm{H}$ & $\mathrm{F}$ & TMPNH & $1.45 \pm 0.25$ & $3.90 \pm 0.30$ \\
\hline $14 \mathrm{e}$ & $\mathrm{CH}$ & $\mathrm{CH}$ & $\mathrm{H}$ & $\mathrm{H}$ & PIP & $1.75 \pm 0.25$ & $2.85 \pm 0.05$ \\
\hline $14 f$ & $\mathrm{CH}$ & $\mathrm{CH}$ & $\mathrm{H}$ & $\mathrm{F}$ & PIP & $2.35 \pm 0.05$ & $2.35 \pm 0.15$ \\
\hline $24 a$ & $\mathrm{~N}$ & $\mathrm{CH}$ & PMB & $\mathrm{H}$ & $\mathrm{PhNH}$ & $>10$ & $>10$ \\
\hline $24 b$ & $\mathrm{~N}$ & $\mathrm{CH}$ & PMB & $\mathrm{F}$ & $\mathrm{PhNH}$ & $>10$ & $>10$ \\
\hline $24 c$ & $\mathrm{~N}$ & $\mathrm{CH}$ & PMB & $\mathrm{H}$ & TMPNH & $2.25 \pm 0.35$ & $4.50 \pm 0.25$ \\
\hline $24 d$ & $\mathrm{~N}$ & $\mathrm{CH}$ & PMB & $\mathrm{F}$ & TMPNH & $>10$ & $>10$ \\
\hline $25 a$ & $\mathrm{~N}$ & $\mathrm{CH}$ & PMB & $\mathrm{H}$ & PIP & $>10$ & $>10$ \\
\hline $25 b$ & $\mathrm{~N}$ & $\mathrm{CH}$ & PMB & $\mathrm{F}$ & PIP & $>10$ & $>10$ \\
\hline $26 a$ & $\mathrm{~N}$ & $\mathrm{CH}$ & $\mathrm{H}$ & $\mathrm{H}$ & $\mathrm{PhNH}$ & $6.20 \pm 0.23$ & $2.75 \pm 0.35$ \\
\hline $26 b$ & $\mathrm{~N}$ & $\mathrm{CH}$ & $\mathrm{H}$ & $\mathrm{F}$ & $\mathrm{PhNH}$ & $1.95 \pm 0.05$ & $2.05 \pm 0.05$ \\
\hline $26 c$ & $\mathrm{~N}$ & $\mathrm{CH}$ & $\mathrm{H}$ & $\mathrm{H}$ & TMPNH & $9.70 \pm 0.50$ & $7.80 \pm 0.45$ \\
\hline $26 d$ & $\mathrm{~N}$ & $\mathrm{CH}$ & $\mathrm{H}$ & $\mathrm{F}$ & TMPNH & $5.90 \pm 0.37$ & $8.70 \pm 0.58$ \\
\hline $26 \mathrm{e}$ & $\mathrm{N}$ & $\mathrm{CH}$ & $\mathrm{H}$ & $\mathrm{H}$ & PIP & $>10$ & $>10$ \\
\hline $26 f$ & $\mathrm{~N}$ & $\mathrm{CH}$ & $\mathrm{H}$ & $\mathrm{F}$ & PIP & $>10$ & $>10$ \\
\hline $35 a$ & $\mathrm{~N}$ & $\mathrm{~N}$ & $\mathrm{H}$ & $\mathrm{H}$ & $\mathrm{PhNH}$ & $4.70 \pm 0.18$ & $7.30 \pm 0.62$ \\
\hline $35 b$ & $\mathrm{~N}$ & $\mathrm{~N}$ & $\mathrm{H}$ & $\mathrm{F}$ & $\mathrm{PhNH}$ & $0.55 \pm 0.05$ & $2.30 \pm 0.20$ \\
\hline $35 c$ & $\mathrm{~N}$ & $\mathrm{~N}$ & $\mathrm{H}$ & $\mathrm{H}$ & TMPNH & $5.70 \pm 0.40$ & $4.70 \pm 0.25$ \\
\hline $35 d$ & $\mathrm{~N}$ & $\mathrm{~N}$ & $\mathrm{H}$ & $\mathrm{F}$ & TMPNH & $>10$ & $>10$ \\
\hline $35 e$ & $\mathrm{~N}$ & $\mathrm{~N}$ & $\mathrm{H}$ & $\mathrm{H}$ & PIP & $>10$ & $>10$ \\
\hline $35 \mathrm{f}$ & $\mathrm{N}$ & $\mathrm{N}$ & $\mathrm{H}$ & $\mathrm{F}$ & PIP & $>10$ & $>10$ \\
\hline Dox & & & & & & $0.025 \pm 0.004$ & $0.095 \pm 0.01$ \\
\hline
\end{tabular}

${ }^{a}$ Results presented are means \pm standard deviation (SD) of three independent experiments and are expressed as $\mathrm{IC}_{50}$, i.e., the effective concentration reducing viability by $50 \%$ compared to unexposed control cells; ${ }^{\mathrm{b}} \mathrm{PMB}$ : 4-methoxybenzyl, ${ }^{\mathrm{C}} \mathrm{PhNH}$ : phenylamine, TMPNH: 3,4,5-trimethoxyphenylamine, PIP: 4-methylpiperazin-1-yl. 
Table 2. $\mathrm{IC}_{50}$ values of the most potent compounds in human fibroblasts.

\begin{tabular}{cccc}
\hline Compd. & $\begin{array}{c}\text { WI-38 Fibroblasts } \\
\text { IC }_{\mathbf{5 0}}(\boldsymbol{\mu M})\end{array}$ & $\begin{array}{c}\text { Selectivity Index } \\
\text { PC-3 }\end{array}$ & $\begin{array}{c}\text { Selectivity Index } \\
\text { HCT116 }\end{array}$ \\
\hline $\mathbf{1 4 a}$ & $<10$ & & 222 \\
$\mathbf{1 4 b}$ & $20 \pm 0.4$ & 363 & \\
$\mathbf{1 4} \mathbf{c}$ & $<10$ & & \\
$\mathbf{1 4 d}$ & $<10$ & & 8.3 \\
$\mathbf{1 4}$ & $<10$ & 8.7 & 5.3 \\
$\mathbf{2 6 b}$ & $17 \pm 1.5$ & 22.2 & \\
$\mathbf{3 5 b}$ & $12.2 \pm 1.0$ & &
\end{tabular}

As a general remark, it could be stated that the cytotoxic activity is enhanced in the absence of substituents on the pyrrole or pyrazole nitrogen of the studied compounds and that the pyrrolopyridines possess the most interesting profile, since all six derivatives (14a-f) are potent against both cell lines tested. As already mentioned, $\mathbf{1 4 b}$ is the most active derivative, and at the same time, the analogously substituted pyrrolopyrimidine $\mathbf{2 6} \mathbf{b}$ and pyrazolopyrimidine $\mathbf{3 5 b}$ are also endowed with strong cytotoxic properties. It is of interest to notice that the prostatic cell line appears the most sensitive to the compounds.

The cytotoxicity of the most potent derivatives, i.e., the majority of the pyrrolopyridines $\mathbf{1 4}$, together with $\mathbf{2 6} \mathbf{b}$ and $\mathbf{3 5 b}$, was then examined towards normal human fibroblasts (WI-38); the results are presented in Figure 1 and Table 2.

HCT116 cells
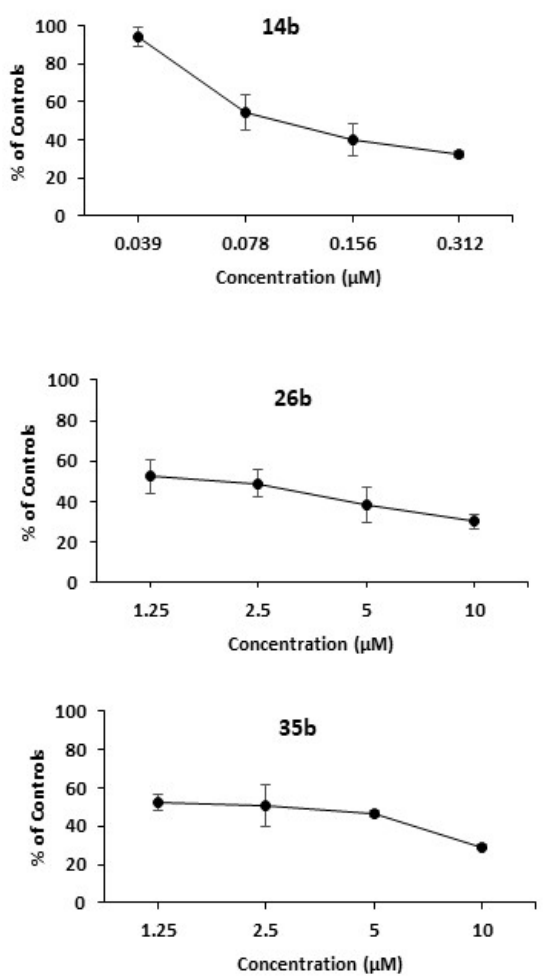

PC-3 cells
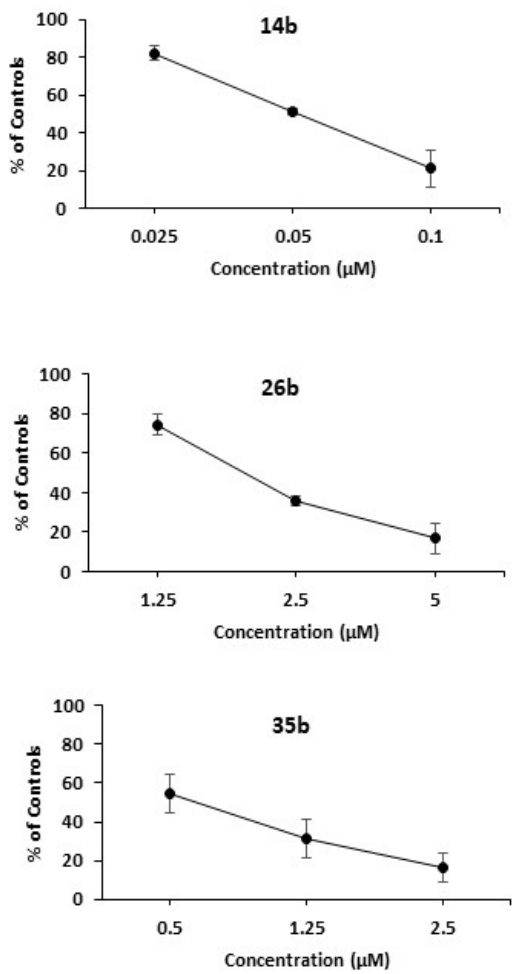

Wl-38 cells
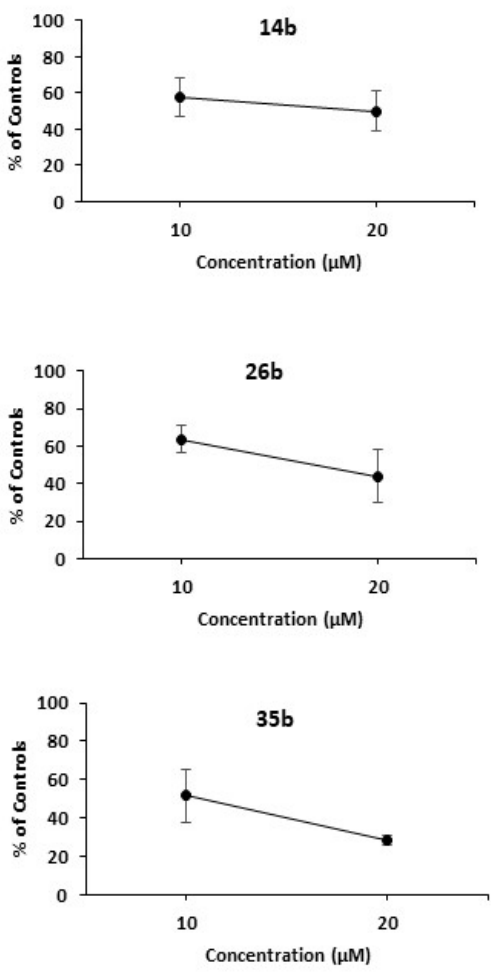

Figure 1. Dose-response curves for the administration of $\mathbf{1 4 b} \mathbf{b} \mathbf{2 6} \mathbf{b}, \mathbf{3 5} \mathbf{b}$ on HCT116, PC-3 and WI-38 cells, as assessed by the MTT assay. Results are expressed as a percentage of controls. Statistical analysis was performed in triplicate determination at $p<0.05$.

Among them, 14a and 14c-e appear to be rather cytotoxic to normal cells. By contrast, it is important to note that the 7-phenylamino substituted compound $\mathbf{1 4 b}$ that bears a 3-(3- 
fluorophenyl)-group, while being highly cytotoxic against both cancer cell lines, appears practically non-toxic against the normal cell line, presenting a selectivity index higher than 200 . The derivatives $\mathbf{2 6} \mathbf{b}$ and $\mathbf{3 5 b}$ that possess the same substitution pattern in the central scaffold retain potent activity against the cancer cell lines and appear non-cytotoxic to the normal cell line as well, albeit to a lesser extent than $\mathbf{1 4 b}$.

Cell-cycle perturbations induced after the incubation of exponentially growing PC3 cells with compounds $\mathbf{1 4 b}, \mathbf{2 6} \mathbf{b}$ and $\mathbf{3 5 b}$ for $72 \mathrm{~h}$ are given in Figure 2 and Table 3. Compound $14 \mathrm{~b}$ caused a statistically significant accumulation of PC-3 cells at $\mathrm{G}_{2} / \mathrm{M}$ phase, significantly reducing, in parallel, the percentage of cells at $\mathrm{G}_{0} / \mathrm{G}_{1}$ phase (the accumulation of cells at $\mathrm{S}$ phase was marginally non-significant).
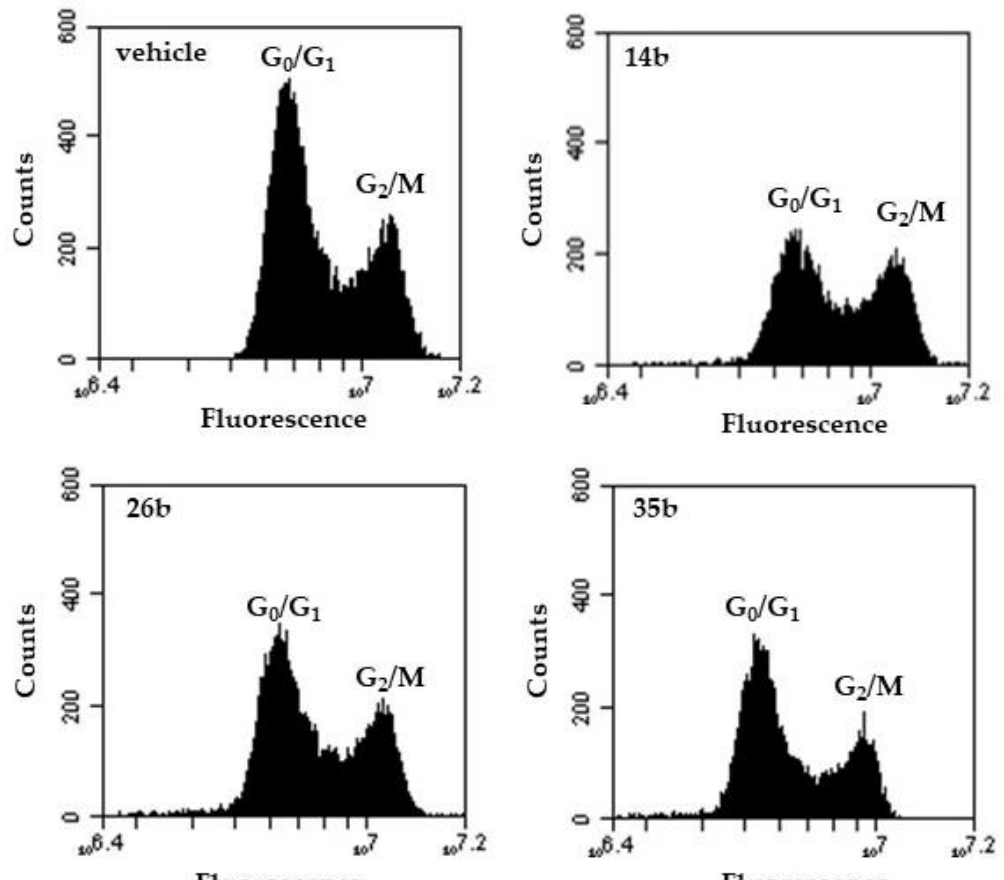

Figure 2. Flow cytometric graphs of DNA content. Indicative DNA content histograms of PC-3 cells treated for $72 \mathrm{~h}$ with $\mathbf{1 4 b}, \mathbf{2 6 b}$ and $\mathbf{3 5 b}$, in comparison to control cells (vehicle). Quantification of cell cycle phase distribution was based on staining by the DNA binding dye propidium iodide.

Table 3. Cell cycle phase distribution at $72 \mathrm{~h}(\%)$.

\begin{tabular}{cccc}
\hline Compound & $\mathbf{G}_{\mathbf{0}} / \mathbf{G}_{\mathbf{1}}$ & $\mathbf{S}$ & $\mathbf{G}_{\mathbf{2}} / \mathbf{M}$ \\
\hline $\mathbf{1 4 b}$ & $48.7( \pm 0.6)$ & $20.5( \pm 1.4)$ & $30.8( \pm 0.8)$ \\
26b & $54.4( \pm 1.1)$ & $20.7( \pm 02.7)$ & $24.9( \pm 1.6)$ \\
35b & $58.9( \pm 0.6)$ & $16.3( \pm 1.8)$ & $24.9( \pm 2.4)$ \\
vehicle & $57.7( \pm 1.3)$ & $17.6( \pm 0.1)$ & $24.8( \pm 1.5)$ \\
\hline
\end{tabular}

Doxorubicin, which was used as a positive control at a concentration of $25 \mathrm{nM}$, showed the expected G2/M phase blockade (58\%) and the induction of apoptosis $(28 \%)$, as previously reported [40]. Furthermore, $\mathbf{1 4 b}, \mathbf{2 6} \mathbf{b}$ and $\mathbf{3 5 b}$ induced apoptosis in PC-3 cells after $72 \mathrm{~h}$ of treatment, as estimated by AnnexinV-7AAD staining. In particular, compound $\mathbf{1 4 b}$ induced the appearance of $26.5 \%( \pm 0.47)$ apoptotic nuclei, $26 \mathbf{b}$ induced the appearance of $17.7 \%( \pm 1.8)$ apoptotic nuclei and $35 \mathrm{~b}$ induced the appearance of $11.5 \%( \pm 0.19)$ apoptotic nuclei, compared to $3.5 \%( \pm 0.05)$ induced by the vehicle (Figure 3$)$. These data give an indication that the mechanism of action of compound $\mathbf{1 4 b}$ may include blocking of the cell cycle at the $\mathrm{G}_{2} / \mathrm{M}$ phase and inducing apoptosis. 


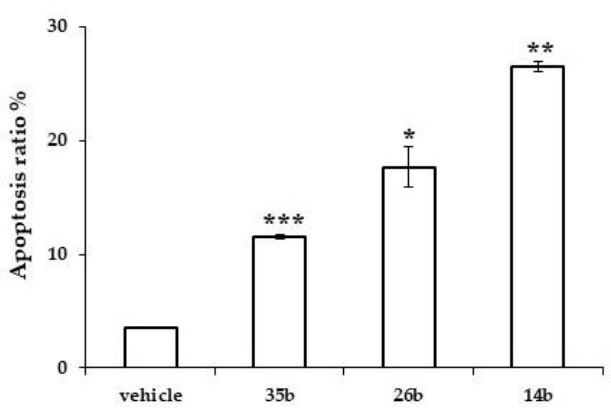

Figure 3. Induction of apoptosis by the test compounds. Percentage of apoptotic nuclei in PC-3 cells treated for $72 \mathrm{~h}$ with the indicated compounds or with vehicle. Bars represent the mean of two independent experiments (error bars: SD); ${ }^{*}$ indicates $p<0.05,{ }^{* *}$ indicates $p<0.005,{ }^{* * *}$ indicates $p<0.001$ (Student's $t$-test)

\section{Materials and Methods}

\subsection{General Information}

The reagents and solvents were purchased from Sigma-Aldrich Chemical Co. (Darmstadt, Germany) or Fluorochem (Derbyshire's Peak District, UK). Reagents were used without further purification. Concerning the dry solvents, methanol and dimethylformamide were dried over 3A and 4A molecular sieves, respectively; toluene was pre-dried using $\mathrm{CaH}_{2}$ and then placed over sodium. Hydrazine is considered a suspect carcinogen in humans, may cause serious damage and should be handled carefully. Melting points were determined on a Büchi apparatus (Flawil, Switzerland) and are uncorrected. ${ }^{1} \mathrm{HNMR}$ spectra and 2D spectra were recorded on a Bruker Avance III 600 or a Bruker Avance DRX 400 instrument (Bruker BioSpin, Baden-Württemberg, Germany), whereas ${ }^{13} \mathrm{CNMR}$ spectra were recorded on a Bruker Avance III 600 spectrometer in deuterated solvents and were referenced to TMS ( $\delta$ scale). The signals of ${ }^{1} \mathrm{H}$ and ${ }^{13} \mathrm{C}$ spectra were unambiguously assigned by using 2D NMR techniques: ${ }^{1} \mathrm{H}^{1} \mathrm{H}$ COSY, NOESY, HMQC and HMBC. Mass spectra were recorded with a LTQ Orbitrap Discovery instrument (Thermo Scientific, Dreieich, Germany), possessing an Ionmax ionization source. Flash chromatography was performed on Merck silica gel $60(0.040-0.063 \mathrm{~mm})$. The purity of all the target compounds that underwent biological evaluation was $>95 \%$, as ascertained by elemental analysis. Elemental analyses were undertaken using a PerkinElmer PE 240C elemental analyzer (Norwalk, CT, U.S.) and the measured values for $\mathrm{C}, \mathrm{H}$ and $\mathrm{N}$ were within $\pm 0.4 \%$ of the theoretical values. Analytical thin layer chromatography (TLC) was carried out on precoated $(0.25 \mathrm{~mm})$ Merck silica gel F-254 plates (Merck KGa, Darmstadt, Germany). The controlled injection of solutions was performed with the Bioblock Scientific device (Illkirch, France).

\subsection{Synthesis}

7-Chloro-3-iodo- $1 H$-pyrrolo[2,3-c]pyridine (7): This compound is mentioned in a patent [41]; here, we provide the methodology and identification data. To a solution of $6(1.40 \mathrm{~g}, 9.15 \mathrm{mmol})$ in anhydrous methanol $(50 \mathrm{~mL}), N$-iodosuccinimide $(2.80 \mathrm{~g}, 12.4$ $\mathrm{mmol}$ ) was added, and the mixture was stirred at $\mathrm{rt}$ for $1 \mathrm{~h}$. Then, the organic solvent was evaporated under vacuo, and the residue was extracted with ethyl acetate. The organic layer was washed with a $10 \%$ sodium thiosulfate aqueous solution, dried $\left(\mathrm{Na}_{2} \mathrm{SO}_{4}\right)$ and evaporated to dryness to result in 7 (2.56 g, 99\%), m.p. $278-279{ }^{\circ} \mathrm{C}\left(\mathrm{CH}_{2} \mathrm{Cl}_{2} / \mathrm{Et}_{2} \mathrm{O}\right) .{ }^{1} \mathrm{H}$ NMR (600 MHz, DMSO- $d_{6}$ ) $\delta$ 12.56-12.40 (brs, 1H, NH), 8.00 (d, J = 5.4Hz, 1H, H-5), 7.89 (s, $1 \mathrm{H}, \mathrm{H}-2), 7.31$ (d, $J=5.4 \mathrm{~Hz}, 1 \mathrm{H}, \mathrm{H}-4) .{ }^{13} \mathrm{C}$ NMR (151 MHz, DMSO-d $\left.{ }_{6}\right) \delta 137.95$ (C-5), 136.50 (C-3a), 134.66 (C-2), 133.72 (C-7), 129.68 (C-7a), 114.63 (C-4), 56.54 (C-3). HR-MS (ESI) $m / z$ : Calcd for $\mathrm{C}_{7} \mathrm{H}_{5} \mathrm{ClIN}_{2}:[\mathrm{M}+\mathrm{H}]^{+}=278.9181$, found 278.9193 .

7-Chloro-3-iodo-1-(4-methoxybenzyl)-1H-pyrrolo[2,3-c]pyridine (8): To a solution of $7(2.40 \mathrm{~g}, 8.62 \mathrm{mmol})$ in anhydrous $N, N$-dimethylformamide $(10 \mathrm{~mL})$, sodium hydride (300 mg, 60\% in hexanes) was added under cooling in an argon atmosphere, and the 
resulting solution was stirred at rt for $15 \mathrm{~min}$. Then, 4-methoxybenzyl chloride (1.40 $\mathrm{mL}, 10.4 \mathrm{mmol}$ ) was added, and the solution was stirred at $\mathrm{rt}$ for $90 \mathrm{~min}$. The solvent was vacuum-evaporated, ice-water was added to the residue and the precipitate was filtered and air-dried to provide pure 8 (3.40 g, 99\%), m.p. $98-99{ }^{\circ} \mathrm{C}\left(\mathrm{CH}_{2} \mathrm{Cl}_{2} / \mathrm{Et}_{2} \mathrm{O}\right) .{ }^{1} \mathrm{H} \mathrm{NMR}(600 \mathrm{MHz}$, DMSO- $\left.d_{6}\right) \delta 8.06(\mathrm{~s}, 1 \mathrm{H}, \mathrm{H}-2), 8.02(\mathrm{~d}, J=5.3 \mathrm{~Hz}, 1 \mathrm{H}, \mathrm{H}-5), 7.34(\mathrm{~d}, J=5.3 \mathrm{~Hz}, 1 \mathrm{H}, \mathrm{H}-4), 7.04$ $(\mathrm{d}, J=8.6 \mathrm{~Hz}, 2 \mathrm{H}$, methoxybenzyl H-2,6), $6.87(\mathrm{~d}, J=8.6 \mathrm{~Hz}, 2 \mathrm{H}$, methoxybenzyl H-3,5), 5.72 (s, 2H, $\mathrm{CH}_{2}$ ), 3.69 (s, 3H, $\left.\mathrm{CH}_{3} \mathrm{O}\right) .{ }^{13} \mathrm{C} \mathrm{NMR}\left(151 \mathrm{MHz}, \mathrm{DMSO}-d_{6}\right) \delta 158.63$ (methoxybenzyl C-4), 139.37 (C-2), 138.56 (C-5), 138.22 (C-3a), 132.76 (C-7), 130.14 (methoxybenzyl C-1), 128.39 (C-7a), 127.66 (methoxybenzyl C-2,6), 115.34 (C-4), 114.08 (methoxybenzyl C-3,5), 56.36 (C-3), $55.03\left(\mathrm{CH}_{3} \mathrm{O}\right), 50.60\left(\mathrm{CH}_{2}\right)$. HR-MS (ESI) $m / z$ : Calcd for $\mathrm{C}_{15} \mathrm{H}_{13} \mathrm{ClIN}_{2} \mathrm{O}$ : $[\mathrm{M}+$ $\mathrm{H}]^{+}=398.9756$, found 398.9766 .

7-Chloro-1-(4-methoxybenzyl)-3-phenyl-1H-pyrrolo[2,3-c]pyridine (9a): To a solution of $8(1.00 \mathrm{~g}, 2.51 \mathrm{mmol})$ in toluene $(40 \mathrm{~mL})$ and absolute ethanol $(5 \mathrm{~mL})$, phenylboronic acid (360 mg, $2.95 \mathrm{mmol})$, potassium carbonate $(700 \mathrm{mg}, 5.07 \mathrm{mmol}$ ) and tetrakis(triphenylphos phine)palladium $(70 \mathrm{mg}, 60.6 \mu \mathrm{mol})$ were added sequentially, and the solution was heated at reflux for $3 \mathrm{~h}$. The solvents were evaporated under vacuo, water was added to the residue and it was extracted with ethyl acetate. The organic layer was dried $\left(\mathrm{Na}_{2} \mathrm{SO}_{4}\right)$ and concentrated to dryness, and the residue was purified by silica gel column chromatography (cyclohexane/ethyl acetate: $4 / 1)$ to give $9 a(770 \mathrm{mg}, 88 \%)$, m.p. $85-86{ }^{\circ} \mathrm{C}\left(\mathrm{CHCl}_{3} / n\right.$-hexane). ${ }^{1} \mathrm{H}$ NMR $\left(600 \mathrm{MHz}, \mathrm{CDCl}_{3}\right) \delta 8.08$ (d, $\left.J=5.5 \mathrm{~Hz}, 1 \mathrm{H}, \mathrm{H}-5\right), 7.78$ (d, J=5.5 Hz, 1H, H-4), $7.55(\mathrm{~d}, J=7.4 \mathrm{~Hz}, 2 \mathrm{H}$, phenyl H-2,6), 7.47-7.44 (m, 3H, H-2, phenyl H-3,5), 7.34 (t, $J=7.4$ $\mathrm{Hz}, 1 \mathrm{H}$, phenyl H-4), $7.11(\mathrm{~d}, J=8.7 \mathrm{~Hz}, 2 \mathrm{H}$, methoxybenzyl H-2,6), 6.87 (d, J = 8.7 Hz, 2H, methoxybenzyl H-3,5), $5.78\left(\mathrm{~s}, 2 \mathrm{H}, \mathrm{CH}_{2}\right), 3.78\left(\mathrm{~s}, 3 \mathrm{H}, \mathrm{CH}_{3} \mathrm{O}\right) .{ }^{13} \mathrm{C} \mathrm{NMR}(151 \mathrm{MHz}$, $\mathrm{CDCl}_{3}$ ) $\delta 159.62$ (methoxybenzyl C-4), 137.03 (C-5), 135.24 (C-7), 133.57 (phenyl C-1), 133.25 (C-3a), 132.74 (C-2), 129.42 (methoxybenzyl C-1), 129.21 (phenyl C-3,5), 129.13 (C-7a), 128.45 (methoxybenzyl C-2,6), 127.73 (phenyl C-2,6), 127.23 (phenyl C-4), 118.25 (C-3), 114.57 (methoxybenzyl C-3,5), $114.55(\mathrm{C}-4), 55.46\left(\mathrm{CH}_{3} \mathrm{O}\right), 51.80\left(\mathrm{CH}_{2}\right)$. HR-MS (ESI) $\mathrm{m} / z$ : Calcd for $\mathrm{C}_{21} \mathrm{H}_{18} \mathrm{ClN}_{2} \mathrm{O}$ : $[\mathrm{M}+\mathrm{H}]^{+}=349.1103$, found 349.1115 .

7-Chloro-3-(3-fluorophenyl)-1-(4-methoxybenzyl)-1H-pyrrolo[2,3-c]pyridine (9b): This compound was synthesized using a procedure analogous to that of $\mathbf{9 a}$, starting from $\mathbf{8}$. Purification was carried out by silica gel column chromatography (cyclohexane/ethyl acetate: 7/3). Oil, yield: $98 \% .{ }^{1} \mathrm{H}$ NMR $\left(600 \mathrm{MHz}, \mathrm{CDCl}_{3}\right) \delta 8.11(\mathrm{~d}, J=5.5 \mathrm{~Hz}, 1 \mathrm{H}, \mathrm{H}-5), 7.75(\mathrm{~d}, J=$ $5.5 \mathrm{~Hz}, 1 \mathrm{H}, \mathrm{H}-4)$, 7.45-7.41 (m, 2H, H-2, fluorophenyl H-5), 7.38-7.34 (m, 1H, fluorophenyl H-6), 7.29-7.26 (m, 1H, fluorophenyl H-2), $7.14(\mathrm{~d}, \mathrm{~J}=8.9 \mathrm{~Hz}, 2 \mathrm{H}$, methoxybenzyl H-2,6), 7.06-7.01 (m, 1H, fluorophenyl H-4), $6.90(\mathrm{~d}, J=8.9 \mathrm{~Hz}, 2 \mathrm{H}$, methoxybenzyl H-3,5), 5.80 (s, $\left.2 \mathrm{H}, \mathrm{CH}_{2}\right), 3.82\left(\mathrm{~s}, 3 \mathrm{H}, \mathrm{CH}_{3} \mathrm{O}\right) .{ }^{13} \mathrm{C} \mathrm{NMR}\left(151 \mathrm{MHz}, \mathrm{CDCl}_{3}\right) \delta 164.20,162.57$ (fluorophenyl C-3), 159.55 (methoxybenzyl C-4), 138.61 (C-5), 134.48, 134.41 (fluorophenyl C-1), 134.24 (C-7), 131.72 (C-2), 130.64, 130.58 (fluorophenyl C-5), 129.64 (C-7a), 129.37 (methoxybenzyl C-1), 128.55 (C-3a), 128.44 (methoxybenzyl C-2,6), 123.20 (fluorophenyl C-6), 116.70 (C-3), 114.53 (methoxybenzyl C-3,5), 114.43, 114.29 (fluorophenyl C-2), 114.15 (C-4), 113.78, 113.64 (fluorophenyl C-4), $55.44\left(\mathrm{CH}_{3} \mathrm{O}\right), 51.68\left(\mathrm{CH}_{2}\right)$. HR-MS (ESI) $m / z$ : Calcd for $\mathrm{C}_{21} \mathrm{H}_{17} \mathrm{ClFN}_{2} \mathrm{O}$ : $[\mathrm{M}+\mathrm{H}]^{+}=367.1008$, found 367.0995.

1-(4-Methoxybenzyl)-N,3-diphenyl-1H-pyrrolo[2,3-c]pyridin-7-amine (10a): To a solution of $9 \mathbf{a}(120 \mathrm{mg}, 0.34 \mathrm{mmol})$ in anhydrous toluene $(2 \mathrm{~mL})$, tris(dibenzylideneacetone)dipa lladium(0) (8.0 mg, $8.7 \mu \mathrm{mol})$, 2,2'-bis(diphenylphosphino)-1,1'-dinapthalene (16 mg, 0.26 $\mathrm{mmol})$, potassium tert-butoxide $(60 \mathrm{mg}, 0.50 \mathrm{mmol})$ and aniline $(46 \mu \mathrm{L}, 0.50 \mathrm{mmol})$ were added sequentially, and the solution was heated at reflux for $3.5 \mathrm{~h}$. It was then extracted with ethyl acetate, the organic layer was dried $\left(\mathrm{Na}_{2} \mathrm{SO}_{4}\right)$ and concentrated to dryness, and the residue was purified by silica gel column chromatography (cyclohexane/ethyl acetate: $1 / 1)$ to give 10a in $87 \%$ yield, m.p. $180-181{ }^{\circ} \mathrm{C}\left(\mathrm{EtOAc} / \mathrm{Et}_{2} \mathrm{O}\right) .{ }^{1} \mathrm{H} \mathrm{NMR}(600 \mathrm{MHz}$, Acetone$\left.d_{6}\right) \delta 7.87(\mathrm{~d}, J=5.3 \mathrm{~Hz}, 1 \mathrm{H}, \mathrm{H}-5), 7.81(\mathrm{~s}, 1 \mathrm{H}, \mathrm{H}-2), 7.70(\mathrm{~d}, J=7.5 \mathrm{~Hz}, 2 \mathrm{H}, 3$-phenyl H-2,6), 7.49-7.44 (m, 3H, H-4, 3-phenyl H-3,5), 7.29 (t, $J=7.4$ Hz, 1H, 3-phenyl H-4), 7.25-7.18 (m, $6 \mathrm{H}, \mathrm{N}^{7}$-phenyl H-2,6, methoxybenzyl H-2,6, N7 -phenyl H-3,5), 7.16 (brs, 1H, NH), 6.92 $(\mathrm{d}, J=8.7 \mathrm{~Hz}, 2 \mathrm{H}$, methoxybenzyl H-3,5), 6.87 (t, J = 7.0 Hz, 1H, N7 -phenyl H-4), 5.69 (s, 
$\left.2 \mathrm{H}, \mathrm{CH}_{2}\right), 3.75\left(\mathrm{~s}, 3 \mathrm{H}, \mathrm{CH}_{3} \mathrm{O}\right) .{ }^{13} \mathrm{C}$ NMR $\left(151 \mathrm{MHz}\right.$, Acetone- $\left.d_{6}\right) \delta 160.62$ (methoxybenzyl C-4), 144.01 (N7 -phenyl C-1), 143.60 (C-7), 138.19 (C-5), 135.83 (3-phenyl C-1), 134.39 (C-3a), 131.95 (C-2), 131.25 (methoxybenzyl C-1), 129.81 (3-phenyl C-3,5), 129.52 (N7-phenyl C-3,5), 128.94 (methoxybenzyl C-2,6), 128.02 (3-phenyl C-2,6), 126.99 (3-phenyl C-4), 124.93 (C-7a), 121.51 ( $\mathrm{N}^{7}$-phenyl C-4), 119.09 ( $\mathrm{N}^{7}$-phenyl C-2,6), 117.51 (C-3), 115.41 (methoxybenzyl C-3,5), $110.05(\mathrm{C}-4), 55.68\left(\mathrm{CH}_{3} \mathrm{O}\right), 52.53\left(\mathrm{CH}_{2}\right)$. HR-MS (ESI) $\mathrm{m} / z$ : Calcd for $\mathrm{C}_{27} \mathrm{H}_{24} \mathrm{~N}_{3} \mathrm{O}$ : [M $+\mathrm{H}^{+}=406.1914$, found 406.1900. Anal. Calcd for $\mathrm{C}_{27} \mathrm{H}_{23} \mathrm{~N}_{3} \mathrm{O}: \mathrm{C}, 79.97 ; \mathrm{H}, 5.72 ; \mathrm{N}, 10.36$. Found: C, 80.06; H, 5.77; N, 10.21.

3-(3-Fluorophenyl)-1-(4-methoxybenzyl)- $N$-phenyl-1H-pyrrolo[2,3-c]pyridin-7-amine (10b): This compound was synthesized using a procedure analogous to that of 10a, starting from 9b. Purification was carried out by silica gel column chromatography (dichloromethane/ethyl acetate: 9/1). Yield: 91\%, m.p. $165-166{ }^{\circ} \mathrm{C}\left(\mathrm{CH}_{2} \mathrm{Cl}_{2} / n\right.$-pentane). ${ }^{1} \mathrm{H}$ NMR $\left(600 \mathrm{MHz}\right.$, Acetone- $\left.d_{6}\right) \delta 7.91$ (s, 1H, H-2), 7.89 (d, $\left.J=5.4 \mathrm{~Hz}, 1 \mathrm{H}, \mathrm{H}-5\right), 7.58$ 7.55 (m, 1H, fluorophenyl H-6), 7.51-7.48 (m, 2H, H-4, fluorophenyl H-5), 7.47-7.43 (m, $1 \mathrm{H}$, fluorophenyl H-2), 7.25-7.16 (m, 7H, phenyl H-2,6, methoxybenzyl H-2,6, phenyl H3,5,NH), 7.07-7.03 (m, 1H, fluorophenyl H-4), $6.92(\mathrm{~d}, J=8.2 \mathrm{~Hz}, 2 \mathrm{H}$, methoxybenzyl H-3,5), $6.88\left(\mathrm{t}, J=7.0 \mathrm{~Hz}, 1 \mathrm{H}\right.$, phenyl H-4), $5.71\left(\mathrm{~s}, 2 \mathrm{H}, \mathrm{CH}_{2}\right), 3.76\left(\mathrm{~s}, 3 \mathrm{H}, \mathrm{CH}_{3} \mathrm{O}\right) .{ }^{13} \mathrm{C} \mathrm{NMR}(151$ $\mathrm{MHz}$, Acetone- $d_{6}$ ) $\delta 165.09,163.48$ (fluorophenyl C-3), 160.67 (methoxybenzyl C-4), 143.89 (phenyl C-1), 143.70 (C-7), 138.51 (C-5), 138.32 (fluorophenyl C-1), 134.16 (C-3a), 132.59 (C-2), 131.65, 131.59 (fluorophenyl C-5), 131.07 (methoxybenzyl C-1), 129.52 (phenyl C-3,5), 128.96 (methoxybenzyl C-2,6), 124.90 (C-7a), 123.80 (fluorophenyl C-6), 121.61 (phenyl C-4), 119.18 (phenyl C-2,6), 116.21 (C-3), 115.44 (methoxybenzyl C-3,5), 114.35, 114.21 (fluorophenyl C-2), 113.52, 113.38 (fluorophenyl C-4), 109.81 (C-4), $55.69\left(\mathrm{CH}_{3} \mathrm{O}\right), 52.65\left(\mathrm{CH}_{2}\right)$. HR-MS (ESI) $m / z$ : Calcd for $\mathrm{C}_{27} \mathrm{H}_{23} \mathrm{FN}_{3} \mathrm{O}:[\mathrm{M}+\mathrm{H}]^{+}=424.1820$, found 424.1804. Anal. Calcd for $\mathrm{C}_{27} \mathrm{H}_{22} \mathrm{FN}_{3} \mathrm{O}: \mathrm{C}, 76.58 ; \mathrm{H}, 5.24 ; \mathrm{N}, 9.92$. Found: $\mathrm{C}, 76.73 ; \mathrm{H}, 5.29 ; \mathrm{N}, 9.66$.

1-(4-Methoxybenzyl)-N-(3,4,5-trimethoxyphenyl)-3-phenyl-1H-pyrrolo[2,3-c]pyridin7-amine (10c): This compound was synthesized using a procedure analogous to that of 10a, starting from 9a. Purification was carried out by silica gel column chromatography (cyclohexane/ethyl acetate: 1/1). Yield: 60\%, m.p. $136-137{ }^{\circ} \mathrm{C}\left(\mathrm{EtOAc} / \mathrm{Et}_{2} \mathrm{O}\right) .{ }^{1} \mathrm{H} \mathrm{NMR}$ $\left(600 \mathrm{MHz}, \mathrm{DMSO}-d_{6}\right) \delta 7.99$ (s, 1H, H-2), 7.89 (s, 1H, NH), 7.82 (d, J = 5.5 Hz, 1H, H-5), 7.66 $(\mathrm{d}, J=7.5 \mathrm{~Hz}, 2 \mathrm{H}$, phenyl H-2,6), $7.46(\mathrm{t}, J=7.6 \mathrm{~Hz}, 2 \mathrm{H}$, phenyl H-3,5), $7.42(\mathrm{~d}, J=5.5 \mathrm{~Hz}$, $1 \mathrm{H}, \mathrm{H}-4), 7.28(\mathrm{t}, J=7.3 \mathrm{~Hz}, 1 \mathrm{H}$, phenyl H-4), $7.10(\mathrm{~d}, J=8.5 \mathrm{~Hz}, 2 \mathrm{H}$, methoxybenzyl H-2,6), $6.83(\mathrm{~d}, J=8.5 \mathrm{~Hz}, 2 \mathrm{H}$, methoxybenzyl H-3,5), 6.54 (s, 2H, trimethoxyphenyl H-2,6), 5.65 (s, $\left.2 \mathrm{H}, \mathrm{CH}_{2}\right), 3.69\left(\mathrm{~s}, 6 \mathrm{H}\right.$, trimethoxyphenyl $\left.\mathrm{CH}_{3} \mathrm{O}-3,5\right), 3.66\left(\mathrm{~s}, 3 \mathrm{H}\right.$, methoxybenzyl $\left.\mathrm{CH}_{3} \mathrm{O}\right)$, 3.61 (s, 3H, trimethoxyphenyl $\left.\mathrm{CH}_{3} \mathrm{O}-4\right) .{ }^{13} \mathrm{C}$ NMR $\left(151 \mathrm{MHz}\right.$, DMSO- $\left.d_{6}\right) \delta 158.66$ (methoxybenzyl C-4), 152.75 (trimethoxyphenyl C-3,5), 142.70 (C-7), 138.92 (trimethoxyphenyl C-1), 137.02 (C-5), 134.34 (phenyl C-1), 132.77 (C-3a), 131.59 (trimethoxyphenyl C-4), 131.46 (C-2), 130.23 (methoxybenzyl C-1), 128.92 (phenyl C-3,5), 128.13 (methoxybenzyl C-2,6), 126.59 (phenyl C-2,6), 125.92 (phenyl C-4), 123.40 (C-7a), 115.51 (C-3), 114.07 (methoxybenzyl C-3,5), 108.67 (C-4), 96.24 (trimethoxyphenyl C-2,6), 60.12 (trimethoxyphenyl $\mathrm{CH}_{3} \mathrm{O}-4$ ), 55.64 (trimethoxyphenyl $\left.\mathrm{CH}_{3} \mathrm{O}-3,5\right), 55.00$ (methoxybenzyl $\mathrm{CH}_{3} \mathrm{O}$ ), $51.06\left(\mathrm{CH}_{2}\right)$. HR-MS (ESI) $m / z$ : Calcd for $\mathrm{C}_{30} \mathrm{H}_{30} \mathrm{~N}_{3} \mathrm{O}_{4}:[\mathrm{M}+\mathrm{H}]^{+}=496.2231$, found 496.2224. Anal. Calcd for $\mathrm{C}_{30} \mathrm{H}_{29} \mathrm{~N}_{3} \mathrm{O}_{4}$ : C, 72.71; H, 5.90; N, 8.48. Found: $\mathrm{C}, 72.80 ; \mathrm{H}, 5.94 ; \mathrm{N}, 8.34$.

3-(3-Fluorophenyl)-1-(4-methoxybenzyl)- N-(3,4,5-trimethoxyphenyl)-1H-pyrrolo[2,3c]pyridin-7-amine (10d): This compound was synthesized using a procedure analogous to that of $\mathbf{1 0 a}$, starting from $\mathbf{9 b}$. The reaction was completed at $20 \mathrm{~h}$. Purification was carried out by silica gel column chromatography (cyclohexane/ethyl acetate: $1 / 1$ ). Yield: 60\%, m.p. 93-94 ${ }^{\circ} \mathrm{C}\left(\mathrm{CH}_{2} \mathrm{Cl}_{2} / n\right.$-pentane). ${ }^{1} \mathrm{H}$ NMR (600 MHz, Acetone- $\left.d_{6}\right) \delta 7.91(\mathrm{~s}, 1 \mathrm{H}, \mathrm{H}-2), 7.87(\mathrm{~d}$, $J=5.2 \mathrm{~Hz}, 1 \mathrm{H}, \mathrm{H}-5), 7.56-7.54$ (m, 1H, fluorophenyl H-6), 7.49-7.46 (m, 1H, fluorophenyl $\mathrm{H}-5)$, 7.45-7.42 (m, 2H, H-4, fluorophenyl H-2), 7.26 (d, $J=8.8 \mathrm{~Hz}, 2 \mathrm{H}$, methoxybenzyl H-2,6), 7.07-7.02 (m, 1H, fluorophenyl H-4), 6.98 (d, J = 8.8 Hz, 2H, methoxybenzyl H-3,5), $6.58(\mathrm{~s}, 2 \mathrm{H}$, trimethoxyphenyl $\mathrm{H}-2,6), 5.74\left(\mathrm{~s}, 2 \mathrm{H}, \mathrm{CH}_{2}\right), 3.77\left(\mathrm{~s}, 3 \mathrm{H}\right.$, methoxybenzyl $\left.\mathrm{CH}_{3} \mathrm{O}\right)$, 3.73 (s, 6H, trimethoxyphenyl $\left.\mathrm{CH}_{3} \mathrm{O}-3,5\right), 3.66\left(\mathrm{~s}, 3 \mathrm{H}\right.$, trimethoxyphenyl $\left.\mathrm{CH}_{3} \mathrm{O}-4\right) .{ }^{13} \mathrm{C}$ NMR (151 MHz, Acetone- $d_{6}$ ) $\delta$ 165.08, 163.47 (fluorophenyl C-3), 160.76 (methoxyben- 
zyl C-4), 154.40 (trimethoxyphenyl C-3,5), 143.99 (C-7), 139.50 (trimethoxyphenyl C-1), 138.38, 138.32 (fluorophenyl C-1), 133.95 (trimethoxyphenyl C-4), 133.86 (C-3a), 132.64 (C-2), 131.65, 131.59 (fluorophenyl C-5), 131.01 (methoxybenzyl C-1), 128.92 (methoxybenzyl C-2,6), 124.30 (C-7a), 123.79 (fluorophenyl C-6), 116.18(C-3), 115.56 (methoxybenzyl C-3,5), 114.34, 114.20 (fluorophenyl C-2), 113.51, 113.37 (fluorophenyl C-4), 109.16 (C-4), 97.69 (trimethoxyphenyl C-2,6), 60.71 (trimethoxyphenyl $\mathrm{CH}_{3} \mathrm{O}-4$ ), 56.40 (trimethoxyphenyl $\left.\mathrm{CH}_{3} \mathrm{O}-3,5\right)$, 55.72 (methoxybenzyl $\left.\mathrm{CH}_{3} \mathrm{O}\right), 52.77\left(\mathrm{CH}_{2}\right)$. HR-MS (ESI) $\mathrm{m} / \mathrm{z}$ : Calcd for $\mathrm{C}_{30} \mathrm{H}_{29} \mathrm{FN}_{3} \mathrm{O}_{4}:[\mathrm{M}+\mathrm{H}]^{+}=514.2137$, found 514.2114. Anal. Calcd for $\mathrm{C}_{30} \mathrm{H}_{28} \mathrm{FN}_{3} \mathrm{O}_{4}$ : C, 70.16; H, 5.50; N, 8.18. Found: C, 70.01; H, 5.44; N, 8.37.

7-Chloro-1-(4-methoxybenzyl)-3-phenyl-1H-pyrrolo[2,3-c]pyridin-6-oxide (11a): To a solution of $9 \mathrm{a}(240 \mathrm{mg}, 0.69 \mathrm{mmol})$ in dichloromethane $(5 \mathrm{~mL}), \mathrm{m}$-chloroperoxybenzoic acid (180 mg, $1.04 \mathrm{mmol}$ ) was added, and the solution was stirred at $\mathrm{rt}$ for $72 \mathrm{~h}$. The solvent was then vacuum-evaporated, and a saturated sodium bicarbonate solution was added to the residue and extracted with ethyl acetate. The organic layer was dried $\left(\mathrm{Na}_{2} \mathrm{SO}_{4}\right)$ and concentrated to dryness, and the residue was purified by silica gel column chromatography (ethyl acetate/methanol: 95/5) to yield 11a (220 mg, 88\%), m.p. 160-161 ${ }^{\circ} \mathrm{C}$ (EtOAc/npentane). ${ }^{1} \mathrm{H}$ NMR $\left(600 \mathrm{MHz}, \mathrm{CDCl}_{3}\right) \delta 8.16(\mathrm{~d}, J=6.8 \mathrm{~Hz}, 1 \mathrm{H}, \mathrm{H}-5), 7.60(\mathrm{~d}, J=6.8 \mathrm{~Hz}, 1 \mathrm{H}$, $\mathrm{H}-4), 7.49(\mathrm{~d}, J=7.6 \mathrm{~Hz}, 2 \mathrm{H}$, phenyl H-2,6), $7.43(\mathrm{t}, J=7,5 \mathrm{~Hz}, 2 \mathrm{H}$, phenyl H-3,5), $7.37(\mathrm{~s}, 1 \mathrm{H}$, $\mathrm{H}-2), 7.33(\mathrm{t}, J=7.3 \mathrm{~Hz}, 1 \mathrm{H}$, phenyl H-4), $7.04(\mathrm{~d}, J=7.8 \mathrm{~Hz}, 2 \mathrm{H}$, methoxybenzyl H-2,6), 6.84 $(\mathrm{d}, J=7.8 \mathrm{~Hz}, 2 \mathrm{H}$, methoxybenzyl $\mathrm{H}-3,5), 5.66\left(\mathrm{~s}, 2 \mathrm{H}, \mathrm{CH}_{2}\right), 3.76\left(\mathrm{~s}, 3 \mathrm{H}, \mathrm{CH}_{3} \mathrm{O}\right) .{ }^{13} \mathrm{C}$ NMR $\left(151 \mathrm{MHz}, \mathrm{CDCl}_{3}\right) \delta 159.68$ (methoxybenzyl C-4), 133.45 (C-5), 132.65 (C-2), 132.11 (phenyl C-4), 130.45 (C-7a), 129.28 (phenyl C-1), 128.79 (phenyl C-3,5), 128.23 (methoxybenzyl C-2,6), 127.67 (phenyl C-2,6), 127.50 (C-7), 126.04 (C-3a), 118.83 (C-3), 114.65 (methoxybenzyl C-3,5), 114.47 (methoxybenzyl C-1), $114.03(\mathrm{C}-4), 55.47\left(\mathrm{CH}_{3} \mathrm{O}\right), 51.83\left(\mathrm{CH}_{2}\right)$. HR-MS (ESI) m/z: Calcd for $\mathrm{C}_{21} \mathrm{H}_{18} \mathrm{ClN}_{2} \mathrm{O}_{2}:[\mathrm{M}+\mathrm{H}]^{+}=365.1052$, found 365.1036 .

7-Chloro-3-(3-fluorophenyl)-1-(4-methoxybenzyl)-1H-pyrrolo[2,3-c]pyridin-6-oxide (11b): This compound was synthesized using a procedure analogous to that of $\mathbf{1 1 a}$, starting from $\mathbf{9 b}$. Purification was carried out by silica gel column chromatography (ethyl acetate/methanol: 9/1). Yield: 88\%, m.p. $124-125{ }^{\circ} \mathrm{C}$ (EtOAc/n-pentane). ${ }^{1} \mathrm{H}$ NMR (600 MHz, Acetone- $\left.d_{6}\right) \delta 8.23$ $(\mathrm{d}, J=6.7 \mathrm{~Hz}, 1 \mathrm{H}, \mathrm{H}-5), 7.63(\mathrm{~d}, J=6.7 \mathrm{~Hz}, 1 \mathrm{H}, \mathrm{H}-4), 7.51(\mathrm{td}, J=7.9,4.2 \mathrm{~Hz}, 1 \mathrm{H}$, fluorophenyl H-5), 7.41 (s, 1H, H-2), 7.37 (dt, $J=7.8,1.2 \mathrm{~Hz}, 1 \mathrm{H}$, fluorophenyl H-6), 7.28 (ddd, $J=10.0,2.5$, $1.7 \mathrm{~Hz}, 1 \mathrm{H}$, fluorophenyl H-2), 7.17 (tdd, $J=8.4,2.7,0.9 \mathrm{~Hz}, 1 \mathrm{H}$, fluorophenyl H-4), 7.07 (d, $J$ $=8.1 \mathrm{~Hz}, 2 \mathrm{H}$, methoxybenzyl H-2,6), $6.87(\mathrm{~d}, J=8.1 \mathrm{~Hz}, 2 \mathrm{H}$, methoxybenzyl H-3,5), 5.68 (s, $\left.2 \mathrm{H}, \mathrm{CH}_{2}\right), 3.78\left(\mathrm{~s}, 3 \mathrm{H}, \mathrm{CH}_{3} \mathrm{O}\right) .{ }^{13} \mathrm{C} \mathrm{NMR}\left(151 \mathrm{MHz}\right.$, Acetone- $\left.d_{6}\right) \delta 163.73,162.11$ (fluorophenyl C-3), 159.84 (methoxybenzyl C-4), 136.00, 135.94 (fluorophenyl C-1), 134.63 (C-7), 133.21 (C-5), 132.27 (C-2), 130.23 (C-7a), 130.05, 129.99 (fluorophenyl C-5), 128.24 (methoxybenzyl C-2,6), 125.57 (C-3a), 123.33 (fluorophenyl C-6), 119.10 (C-3), 114.77 (methoxybenzyl C-3,5), 114.47 (methoxybenzyl C-1), 114.43, 114.29 (fluorophenyl C-2), 114.25 (C-4), 114.04, 113.90 (fluorophenyl C-4), $55.48\left(\mathrm{CH}_{3} \mathrm{O}\right), 52.08\left(\mathrm{CH}_{2}\right)$. HR-MS (ESI) $m / z$ : Calcd for $\mathrm{C}_{21} \mathrm{H}_{17} \mathrm{ClFN}_{2} \mathrm{O}_{2}$ : $[\mathrm{M}+\mathrm{H}]^{+}=383.0958$, found 383.0967 .

1-(4-Methoxybenzyl)-7-(4-methylpiperazin-1-yl)-3-phenyl-1H-pyrrolo[2,3-c]pyridine6-oxide (12a): To a solution of 11a $(120 \mathrm{mg}, 0.33 \mathrm{mmol})$ in absolute ethanol (5 mL), 1methylpiperazine $(0.20 \mathrm{~mL}, 2.00 \mathrm{mmol})$ was added, and the solution was heated in an autoclave at $120{ }^{\circ} \mathrm{C}$ for $24 \mathrm{~h}$. The mixture was extracted with ethyl acetate, the organic phase was dried $\left(\mathrm{Na}_{2} \mathrm{SO}_{4}\right)$ and concentrated to dryness and the residue was purified by silica gel column chromatography (dichloromethane/methanol: 8/2) to give 12a (40 mg, $50 \%$ ), m.p. $127-128{ }^{\circ} \mathrm{C}$ (EtOAc/n-pentane). ${ }^{1} \mathrm{H}$ NMR (600 MHz, CD 3 OD) 8.00 (d, J = 6.9 $\mathrm{Hz}, 1 \mathrm{H}, \mathrm{H}-5), 7.85$ (d, J = 6.9 Hz, 1H, H-4), 7.79 (s, 1H, H-2), 7.62 (d, J = 7.7 Hz, 2H, phenyl $\mathrm{H}-2,6), 7.46(\mathrm{t}, J=7.4 \mathrm{~Hz}, 2 \mathrm{H}$, phenyl H-3,5), $7.32(\mathrm{t}, J=7.4 \mathrm{~Hz}, 1 \mathrm{H}$, phenyl H-4), $6.90(\mathrm{~m}, 4 \mathrm{H}$, methoxybenzyl H-2,3,5,6), $5.89\left(\mathrm{~s}, 2 \mathrm{H}, \mathrm{CH}_{2}\right), 4.17-4.14(\mathrm{~m}, 2 \mathrm{H}$, methylpiperazine $\mathrm{H}), 3.75$ $\left(\mathrm{s}, 3 \mathrm{H}, \mathrm{CH}_{3} \mathrm{O}\right), 2.89-2.85(\mathrm{~m}, 2 \mathrm{H}$, methylpiperazine $\mathrm{H}), 2.80-2.75(\mathrm{~m}, 2 \mathrm{H}$, methylpiperazine $\mathrm{H}), 2.40\left(\mathrm{~s}, 3 \mathrm{H}\right.$, methylpiperazine $\left.\mathrm{CH}_{3}\right), 2.24-2.18(\mathrm{~m}, 2 \mathrm{H}$, methylpiperazine $\mathrm{H}) .{ }^{13} \mathrm{C}$ NMR (600 MHz, CD 3 OD) 160.65 (methoxybenzyl C-4), 142.92 (C-7), 135.49 (C-2), 134.34 (phenyl C-1), 134.17 (C-5), 131.90 (methoxybenzyl C-1), 131.56 (C-3a), 130.43 (C-7a), 130.14 (phenyl 
C-3,5), 128.41 (phenyl C-2,6), 128.09 (phenyl C-4), 127.62 (methoxybenzyl C-2,6), 119.51 (C-3), 115.69 (C-4), 115.40 (methoxybenzyl C-3,5), $55.78\left(\mathrm{CH}_{3} \mathrm{O}\right), 55.11$ (methylpiperazine $\mathrm{C}-3,5), 52.30\left(\mathrm{CH}_{2}\right), 47.81$ (methylpiperazine $\left.\mathrm{C}-2,6\right), 45.67$ (methylpiperazine $\mathrm{CH}_{3}$ ). HR-MS (ESI) $m / z$ : Calcd for $\mathrm{C}_{26} \mathrm{H}_{29} \mathrm{~N}_{4} \mathrm{O}_{2}:[\mathrm{M}+\mathrm{H}]^{+}=429.2286$, found 429.2273 .

3-(3-Fluorophenyl)-1-(4-methoxybenzyl)-7-(4-methylpiperazin-1-yl)-1H-pyrrolo[2,3-c] pyridine-6-oxide (12b): This compound was synthesized using a procedure analogous to that of 12a, starting from 11b. Purification was carried out by silica gel column chromatography (dichloromethane/methanol: 85/15). Yield: 50\%, m.p. 91-92 ${ }^{\circ} \mathrm{C}$ (EtOAc/n-pentane). ${ }^{1} \mathrm{H}$ NMR $\left(600 \mathrm{MHz}, \mathrm{CD}_{3} \mathrm{OD}\right) \delta 8.01(\mathrm{~d}, J=6.9 \mathrm{~Hz}, 1 \mathrm{H}, \mathrm{H}-5), 7.85(\mathrm{~d}, J=6.9 \mathrm{~Hz}, 1 \mathrm{H}, \mathrm{H}-4)$, 7.84 (s, 1H, H-2), 7.49-7.45 (m, 1H, fluorophenyl H-5), 7.48-7.44 (m, 1H, fluorophenyl H-6), 7.39-7.34 (m, 1H, fluorophenyl H-2), 7.07-7.01 (m, 1H, fluorophenyl H-4), 6.90-6.88 $(\mathrm{m}$, $4 \mathrm{H}$, methoxybenzyl H-2,3,5,6), $5.91\left(\mathrm{~s}, 2 \mathrm{H}, \mathrm{CH}_{2}\right), 4.14-4.10(\mathrm{~m}, 2 \mathrm{H}$, methylpiperazine $\mathrm{H})$, $3.75\left(\mathrm{~s}, 3 \mathrm{H}, \mathrm{CH}_{3} \mathrm{O}\right), 2.77-2.72(\mathrm{~m}, 4 \mathrm{H}$, methylpiperazine $\mathrm{H}), 2.29(\mathrm{~s}, 3 \mathrm{H}$, methylpiperazine $\left.\mathrm{CH}_{3}\right), 2.10-2.06(\mathrm{~m}, 2 \mathrm{H}$, methylpiperazine $\mathrm{H}) .{ }^{13} \mathrm{C} \mathrm{NMR}\left(151 \mathrm{MHz}, \mathrm{CD}_{3} \mathrm{OD}\right) \delta 165.55,163.93$ (fluorophenyl C-3), 160.63 (methoxybenzyl C-4), 143.34 (C-7), 136.81, 136.76 (fluorophenyl C-1), 135.89 (C-2), 134.46 (C-5), 131.95, 131.89 (fluorophenyl C-5), 131.75 (C-7a), 131.69 (C-3a), 129.72 (methoxybenzyl C-1), 127.66 (methoxybenzyl C-2,6), 124.16 (fluorophenyl C-6), 118.20 (C-3), 115.44 (C-4), 115.37 (methoxybenzyl C-3,5), 114.92, 114.77 (fluorophenyl C-2), 114.66, 114.52 (fluorophenyl C-4), $55.78\left(\mathrm{CH}_{3} \mathrm{O}\right), 55.26$ (methylpiperazine $\left.\mathrm{C}-3,5\right), 52.31$ $\left(\mathrm{CH}_{2}\right), 48.13$ (methylpiperazine C-2,6), 46.10 (methylpiperazine $\mathrm{CH}_{3}$ ). HR-MS (ESI) $\mathrm{m} / \mathrm{z}$ : Calcd for $\mathrm{C}_{26} \mathrm{H}_{28} \mathrm{FN}_{4} \mathrm{O}_{2}:[\mathrm{M}+\mathrm{H}]^{+}=447.2191$, found 447.2176.

1-(4-Methoxybenzyl)-7-(4-methylpiperazin-1-yl)-3-phenyl-1H-pyrrolo[2,3-c]pyridine (13a): To a solution of $12 \mathrm{a}(70 \mathrm{mg}, 0.16 \mathrm{mmol})$ in chloroform $(3 \mathrm{~mL})$, phosphorus trichloride $(0.10 \mathrm{~mL}, 0.67 \mathrm{mmol})$ was added, and the solution was stirred at $\mathrm{rt}$ for $20 \mathrm{~h}$. The solvent was then vacuum-evaporated and the mixture was neutralized with a sodium bicarbonate solution and extracted with ethyl acetate. The organic phase was dried $\left(\mathrm{Na}_{2} \mathrm{SO}_{4}\right)$ and concentrated to dryness, and the residue was purified by silica gel column chromatography (dichloromethane/methanol: 9/1) to give 13a (50 mg, 72\%), m.p. $88-89{ }^{\circ} \mathrm{C}\left(\mathrm{Et}_{2} \mathrm{O} / \mathrm{n}\right.$ pentane). ${ }^{1} \mathrm{H}$ NMR $\left(600 \mathrm{MHz}, \mathrm{CDCl}_{3}\right) 8.04(\mathrm{~d}, J=5.5 \mathrm{~Hz}, 1 \mathrm{H}, \mathrm{H}-5), 7.59-7.54(\mathrm{~m}, 3 \mathrm{H}, \mathrm{H}-4$, phenyl H-2,6), $7.42(\mathrm{t}, J=7.8 \mathrm{~Hz}, 2 \mathrm{H}$, phenyl H-3,5), 7.30-7.27 (m, 2H, H-2, phenyl H-4), $7.08(\mathrm{~d}, J=8.7 \mathrm{~Hz}, 2 \mathrm{H}$, methoxybenzyl H-2,6), $6.83(\mathrm{~d}, J=8.7 \mathrm{~Hz}, 2 \mathrm{H}$, methoxybenzyl $\mathrm{H}-3,5), 5.70\left(\mathrm{~s}, 2 \mathrm{H}, \mathrm{CH}_{2}\right), 3.78\left(\mathrm{~s}, 3 \mathrm{H}, \mathrm{CH}_{3} \mathrm{O}\right), 3.32-3.25$ (brs, $4 \mathrm{H}$, methylpiperazine $\left.\mathrm{H}-2,6\right)$, 3.02-2.57 (brs, 4H, methylpiperazine $\mathrm{H}-3,5), 2.38$ (s, 3H, methylpiperazine $\left.\mathrm{CH}_{3}\right) .{ }^{13} \mathrm{C}$ NMR $\left(600 \mathrm{MHz}, \mathrm{CDCl}_{3}\right) 159.25$ (methoxybenzyl C-4), 150.88 (C-7), 137.69 (C-5), 134.75 (phenyl C-1), 133.87 (C-3a), 130.62 (methoxybenzyl C-1), 129.77 (C-2), 129.02 (phenyl C-3,5), 128.45 (methoxybenzyl C-2,6), 127.51 (phenyl C-2,6), 126.56 (phenyl C-4), 126.49 (C-7a), 118.30 (C-3), 114.37 (methoxybenzyl C-3,5), $111.55(\mathrm{C}-4), 55.47\left(\mathrm{CH}_{3} \mathrm{O}\right), 55.26$ (methylpiperazine C-3,5), 51.14 (methylpiperazine $\mathrm{C}-2,6), 50.38\left(\mathrm{CH}_{2}\right), 46.38$ (methylpiperazine $\left.\mathrm{CH}_{3}\right)$. HR-MS (ESI) $\mathrm{m} / \mathrm{z}$ : Calcd for $\mathrm{C}_{26} \mathrm{H}_{29} \mathrm{~N}_{4} \mathrm{O}:[\mathrm{M}+\mathrm{H}]^{+}=413.2336$, found 413.2319. Anal. Calcd for $\mathrm{C}_{26} \mathrm{H}_{28} \mathrm{~N}_{4} \mathrm{O}: \mathrm{C}, 75.70 ; \mathrm{H}, 6.84 ; \mathrm{N}, 13.58$. Found: C, 75.52; H, 6.69; N, 13.66 .

3-(3-Fluorophenyl)-1-(4-methoxybenzyl)-7-(4-methylpiperazin-1-yl)-1H-pyrrolo[2,3-c] pyridine (13b): This compound was synthesized using a procedure analogous to that of 13a, starting from 12b. Purification was carried out by silica gel column chromatography (dichloromethane/methanol: 9/1). Oil, yield: $99 \% .{ }^{1} \mathrm{H}$ NMR $\left(600 \mathrm{MHz}\right.$, Acetone- $\left.d_{6}\right) \delta$ $7.98(\mathrm{~d}, J=5.6 \mathrm{~Hz}, 1 \mathrm{H}, \mathrm{H}-5), 7.85(\mathrm{~s}, 1 \mathrm{H}, \mathrm{H}-2), 7.57$ (d, $J=5.5 \mathrm{~Hz}, 1 \mathrm{H}, \mathrm{H}-4), 7.52-7.50$ $(\mathrm{m}, 1 \mathrm{H}$, fluorophenyl H-6), 7.47-7.45 (m, 1H, fluorophenyl H-5), 7.41-7.37 $(\mathrm{m}, 1 \mathrm{H}$, fluorophenyl H-2), $7.21(\mathrm{~d}, J=8.5 \mathrm{~Hz}, 2 \mathrm{H}$, methoxybenzyl H-2,6), 7.06-7.02 $(\mathrm{m}, 1 \mathrm{H}$, fluorophenyl H-4), $6.83\left(\mathrm{~d}, J=8.5 \mathrm{~Hz}, 2 \mathrm{H}\right.$, methoxybenzyl H-3,5), $5.76\left(\mathrm{~s}, 2 \mathrm{H}, \mathrm{CH}_{2}\right), 3.72(\mathrm{~s}, 3 \mathrm{H}$, $\mathrm{CH}_{3} \mathrm{O}$ ), 3.27-3.20 (brs, $4 \mathrm{H}$, methylpiperazine $\mathrm{H}-2,6$ ), 2.75-2.50 (brs, $4 \mathrm{H}$, methylpiperazine $\mathrm{H}-3,5), 2.32$ (s, 3H, methylpiperazine $\left.\mathrm{CH}_{3}\right) .{ }^{13} \mathrm{C}$ NMR $\left(151 \mathrm{MHz}\right.$, Acetone- $\left.d_{6}\right) \delta$ 165.04, 163.43 (fluorophenyl C-3), 160.16 (methoxybenzyl C-4), 151.99 (C-7), 138.71 (C-5), 138.32, 138.26 (fluorophenyl C-1), 134.31 (C-3a), 132.02 (C-2), 131.91 (methoxybenzyl C-1), 131.61, 131.55 (fluorophenyl C-5), 129.48 (methoxybenzyl C-2,6), 127.07 (C-7a), 123.70, 123.69 (fluorophenyl C-6), 117.01 (C-3), 114.85 (methoxybenzyl C-3,5), 114.25, 114.10 (fluorophenyl 
C-2), 113.50, 113.36 (fluorophenyl C-4), $111.79(\mathrm{C}-4), 55.83\left(\mathrm{CH}_{3} \mathrm{O}\right), 55.55$ (methylpiperazine C-3,5), 51.88 (methylpiperazine C-2,6), $51.15\left(\mathrm{CH}_{2}\right), 46.44$ (methylpiperazine $\left.\mathrm{CH}_{3}\right)$. HR-MS (ESI) $\mathrm{m} / \mathrm{z}$ : Calcd for $\mathrm{C}_{26} \mathrm{H}_{28} \mathrm{FN}_{4} \mathrm{O}:[\mathrm{M}+\mathrm{H}]^{+}=431.2242$, found 431.2231. Anal. Calcd for $\mathrm{C}_{26} \mathrm{H}_{27} \mathrm{FN}_{4} \mathrm{O}: \mathrm{C}, 72.54 ; \mathrm{H}, 6.32 ; \mathrm{N}, 13.01$. Found: C, 72.33; H, 6.19; N, 13.26.

N,3-Diphenyl-1H-pyrrolo[2,3-c]pyridin-7-amine (14a): A solution of 10a (50 mg, 0.12 $\mathrm{mmol})$ in trifluoroacetic acid $(2 \mathrm{~mL})$ was heated at $60{ }^{\circ} \mathrm{C}$ for $20 \mathrm{~h}$. The mixture was then vacuum-evaporated, neutralized with a sodium bicarbonate solution, extracted with ethyl acetate, dried $\left(\mathrm{Na}_{2} \mathrm{SO}_{4}\right)$ and concentrated to dryness. The residue was purified by silica gel column chromatography (dichloromethane/methanol: 9/1) to give 14a (yield 99\%), m.p. $139-140{ }^{\circ} \mathrm{C}\left(\mathrm{EtOAc} \mathrm{Et}_{2} \mathrm{O}\right) .{ }^{1} \mathrm{H}$ NMR $\left(600 \mathrm{MHz}\right.$, Acetone- $\left.d_{6}\right) \delta 11.69-11.18$ (brs, $1 \mathrm{H}$, pyrrole $\mathrm{NH})$, 9.45-8.50 (brs, $1 \mathrm{H}$, pyridinamine $\mathrm{NH}), 7.89\left(\mathrm{~d}, J=8.0 \mathrm{~Hz}, 2 \mathrm{H}, \mathrm{N}^{7}\right.$-phenyl H-2,6), 7.82 $(\mathrm{d}, J=5.8 \mathrm{~Hz}, 1 \mathrm{H}, \mathrm{H}-5), 7.77(\mathrm{~s}, 1 \mathrm{H}, \mathrm{H}-2), 7.69$ (d, $J=7.8 \mathrm{~Hz}, 2 \mathrm{H}, 3$-phenyl H-2,6), $7.45(\mathrm{t}, J=$ $7.5 \mathrm{~Hz}, 2 \mathrm{H}, 3$-phenyl H-3,5), $7.39(\mathrm{~d}, J=5.8 \mathrm{~Hz}, 1 \mathrm{H}, \mathrm{H}-4), 7.31\left(\mathrm{t}, J=7.5 \mathrm{~Hz}, 2 \mathrm{H}, \mathrm{N}^{7}\right.$-phenyl $\mathrm{H}-3,5), 7.28$ (t, $J=7.4 \mathrm{~Hz}, 1 \mathrm{H}, 3-$ phenyl H-4), 6.99 (t, $J=7.4 \mathrm{~Hz}, 1 \mathrm{H}, \mathrm{N}^{7}$-phenyl H-4). ${ }^{13} \mathrm{C}$ NMR (151 MHz, Acetone- $\left.d_{6}\right) \delta 143.78$ (C-7), 141.86 (N²-phenyl C-1), 136.08 (C-5), 135.36 (3-phenyl C-1), 131.38 (C-3a), 129.79 (3-phenyl C-3,5), 129.76 ( $\mathrm{N}^{7}$-phenyl C-3,5), 127.93 (3-phenyl C-2,6), 126.95 (3-phenyl C-4), 126.12 ( $\mathrm{N}^{7}$-phenyl C-4), 122.84 (C-2), 122.52 (C-7a), 120.55 ( $\mathrm{N}^{7}$-phenyl C-2,6), 118.92 (C-3), 108.00 (C-4). HR-MS (ESI) $m / z$ : Calcd for $\mathrm{C}_{19} \mathrm{H}_{16} \mathrm{~N}_{3}$ : $[\mathrm{M}+\mathrm{H}]^{+}=286.1339$, found 286.1340. Anal. Calcd for $\mathrm{C}_{19} \mathrm{H}_{15} \mathrm{~N}_{3}: \mathrm{C}, 79.98 ; \mathrm{H}, 5.30 ; \mathrm{N}, 14.72$. Found: C, 79.77; H, 5.16; N, 14.89.

3-(3-Fluorophenyl)- $N$-phenyl-1H-pyrrolo[2,3-c]pyridin-7-amine (14b): This compound was synthesized using a procedure analogous to that of $\mathbf{1 4 a}$, starting from $\mathbf{1 0 b}$. Purification was carried out by silica gel column chromatography (dichloromethane/methanol: 9/1). Yield: $99 \%$, m.p. $168-169{ }^{\circ} \mathrm{C}\left(\mathrm{EtOAc} \mathrm{Et}_{2} \mathrm{O}\right) .{ }^{1} \mathrm{H}$ NMR $\left(600 \mathrm{MHz}\right.$, Acetone- $\left.d_{6}\right) \delta 11.66-11.23$ (brs, 1H, pyrrole NH), 8.94-8.58 (brs, 1H, pyridinamine NH), 7.90-7.85 (m, 4H, H-5, H-2, phenyl H-2,6), 7.57-7.54 (m, 1H, fluorophenyl H-6), 7.51-7.48 (m, 1H, fluorophenyl H-5), 7.46-7.43 (m, 1H, fluorophenyl H-2), $7.40(\mathrm{~d}, J=5.6 \mathrm{~Hz}, 1 \mathrm{H}, \mathrm{H}-4), 7.31(\mathrm{t}, J=7.5 \mathrm{~Hz}, 2 \mathrm{H}$, phenyl H-3,5), 7.06-7.02 (m, 1H, fluorophenyl H-4), $6.98\left(\mathrm{t}, J=7.1 \mathrm{~Hz}, 1 \mathrm{H}\right.$, phenyl H-4). ${ }^{13} \mathrm{C}$ NMR (151 MHz, Acetone- $d_{6}$ ) $\delta$ 165.10, 163.48 (fluorophenyl C-3), 143.94 (C-5, C-7), 142.19 (phenyl C-1), 138.76, 138.70 (fluorophenyl C-1), 136.40 (C-3a), 131.62, 131.56 (fluorophenyl C-5), 131.14 (C-2), 129.69 (phenyl C-3,5), 126.49 (C-7a), 123.69 (phenyl C-4), 122.72, 122.55 (fluorophenyl C-6), 120.22 (phenyl C-2,6), 117.57 (C-3), 114.24, 114.09 (fluorophenyl C-2), 113.44, 113.30 (fluorophenyl C-4), 107.82 (C-4). HR-MS (ESI) m/z: Calcd for $\mathrm{C}_{19} \mathrm{H}_{15} \mathrm{FN}_{3}$ : $[\mathrm{M}+\mathrm{H}]^{+}=304.1245$, found 304.1245. Anal. Calcd for $\mathrm{C}_{19} \mathrm{H}_{14} \mathrm{FN}_{3}: \mathrm{C}, 75.23 ; \mathrm{H}, 4.65 ; \mathrm{N}, 13.85$. Found: C, 75.40; H, 4.71; N, 13.69.

$N$-(3,4,5-trimethoxyphenyl)-3-phenyl-1H-pyrrolo[2,3-c]pyridin-7-amine (14c): This compound was synthesized using a procedure analogous to that of $14 a$, starting from $10 \mathrm{c}$. Purification was carried out by silica gel column chromatography (dichloromethane/methanol: 95/5).Yield: 99\%, m.p. $153-154{ }^{\circ} \mathrm{C}$ (EtOAc/n-pentane). ${ }^{1} \mathrm{H}$ NMR (600 MHz, DMSO- $\left.d_{6}\right) \delta$ 11.56-11.49 (brs, $1 \mathrm{H}$, pyrrole $\mathrm{NH}), 8.73-8.65$ (brs, $1 \mathrm{H}$, pyridinamine $\mathrm{NH}), 7.90(\mathrm{~s}, 1 \mathrm{H}, \mathrm{H}-2), 7.80$ $(\mathrm{d}, J=5.7 \mathrm{~Hz}, 1 \mathrm{H}, \mathrm{H}-5), 7.68(\mathrm{~d}, J=7.6 \mathrm{~Hz}, 2 \mathrm{H}$, phenyl H-2,6), $7.45(\mathrm{t}, J=7.6 \mathrm{~Hz}, 2 \mathrm{H}$, phenyl $\mathrm{H}-3,5), 7.30(\mathrm{~d}, J=5.7 \mathrm{~Hz}, 1 \mathrm{H}, \mathrm{H}-4), 7.28-7.25$ (m, 3H, trimethoxyphenyl H-2,6, phenyl H-4), 3.81 (s, 6H, trimethoxyphenyl $\left.\mathrm{CH}_{3} \mathrm{O}-3,5\right), 3.64\left(\mathrm{~s}, 3 \mathrm{H}\right.$, trimethoxyphenyl $\left.\mathrm{CH}_{3} \mathrm{O}-4\right) .{ }^{13} \mathrm{C}$ NMR $(151$ $\left.\mathrm{MHz}, \mathrm{DMSO}-d_{6}\right) \delta 152.83$ (trimethoxyphenyl C-3,5), 142.93 (C-7), 137.89 (trimethoxyphenyl C-1), 136.24 (C-5), 135.06 (phenyl C-1), 131.83 (trimethoxyphenyl C-4), 129.21 (C-3a), 128.91 (phenyl C-3,5), 126.41 (phenyl C-2,6), 125.70 (phenyl C-4), 124.75 (C-2), 121.31 (C-7a), 116.39 (C-3), 106.62 (C-4), 96.13 (trimethoxyphenyl C-2,6), 60.18 (trimethoxyphenyl $\left.\mathrm{CH}_{3} \mathrm{O}-4\right), 55.76$ (trimethoxyphenyl $\left.\mathrm{CH}_{3} \mathrm{O}-3,5\right)$. HR-MS (ESI) m/z: Calcd for $\mathrm{C}_{22} \mathrm{H}_{20} \mathrm{~N}_{3} \mathrm{O}_{3}$ : $[\mathrm{M}-\mathrm{H}]^{-}=374.1510$, found 374.1508. Anal. Calcd for $\mathrm{C}_{22} \mathrm{H}_{21} \mathrm{~N}_{3} \mathrm{O}_{3}$ : C, 70.38; $\mathrm{H}, 5.64 ; \mathrm{N}, 11.19$. Found: $\mathrm{C}, 70.44 ; \mathrm{H}$, $5.68 ; \mathrm{N}, 11.11$.

3-(3-Fluorophenyl)- $N$-(3,4,5-trimethoxyphenyl)-1H-pyrrolo[2,3-c]pyridin-7-amine (14d): This compound was synthesized using a procedure analogous to that of $\mathbf{1 4 a}$, starting from 10d. Purification was carried out by silica gel column chromatography (dichloromethane/ methanol: 95/5).Yield: 99\%, m.p. ${ }^{149-150}{ }^{\circ} \mathrm{C}$ (EtOAc/n-pentane). ${ }^{1} \mathrm{H} \mathrm{NMR}(600 \mathrm{MHz}$, 
Acetone- $\left.d_{6}\right) \delta 11.52-10,85$ (brs, $1 \mathrm{H}$, pyrrole $\mathrm{NH}$ ), 8.90-8.14 (brs, $1 \mathrm{H}$, pyridinamine $\mathrm{NH}$ ), 7.87 $(\mathrm{d}, J=5.7 \mathrm{~Hz}, 1 \mathrm{H}, \mathrm{H}-5), 7.82$ (s, 1H, H-2), 7.56-7.53 (m, 1H, fluorophenyl H-6), 7.50-7.47 (m, 1H, fluorophenyl H-5), 7.46-7.42 (m, 1H, fluorophenyl H-2), 7.37 (d, J = 5.7 Hz, 1H, $\mathrm{H}-4), 7.27$ (s, 2H, trimethoxyphenyl H-2,6), 7.06-7.02 (m, 1H, fluorophenyl H-4), 3.78 (s, $6 \mathrm{H}$, trimethoxyphenyl $\left.\mathrm{CH}_{3} \mathrm{O}-3,5\right), 3.67$ (s, 3H, trimethoxyphenyl $\left.\mathrm{CH}_{3} \mathrm{O}-4\right) .{ }^{13} \mathrm{C}$ NMR $(151$ $\mathrm{MHz}$, Acetone- $\left.d_{6}\right) \delta 165.08,163.47$ (fluorophenyl C-3), 154.49 (trimethoxyphenyl C-3,5), 144.26 (C-6), 138.81, 138.75 (fluorophenyl C-1), 138.31 (trimethoxyphenyl C-1), 136.86 (C5), 134.33 (trimethoxyphenyl C-4), 131.59, 131.54 (fluorophenyl C-5), 131.03 (C-3a), 126.22 (C-2), 123.65 (fluorophenyl C-6), 122.64 (C-7a), 117.47 (C-3), 114.18, 114.04 (fluorophenyl C-2), 113.38, 113.24 (fluorophenyl C-4), 107.61 (C-4), 98.40 (trimethoxyphenyl C-2,6), 60.73 (trimethoxyphenyl $\mathrm{CH}_{3} \mathrm{O}-4$ ), 56.40 (trimethoxyphenyl $\left.\mathrm{CH}_{3} \mathrm{O}-3,5\right)$. HR-MS (ESI) $\mathrm{m} / z$ : Calcd for $\mathrm{C}_{22} \mathrm{H}_{21} \mathrm{FN}_{3} \mathrm{O}_{3}:[\mathrm{M}+\mathrm{H}]^{+}=394.1562$, found 394.1561. Anal. Calcd for $\mathrm{C}_{22} \mathrm{H}_{20} \mathrm{FN}_{3} \mathrm{O}_{3}: \mathrm{C}$, 67.17; H, 5.12; N, 10.68. Found: C, 67.33; H, 5.24; N, 10.59 .

7-(4-Methylpiperazin-1-yl)-3-phenyl-1H-pyrrolo[2,3-c]pyridine (14e): This compound was synthesized using a procedure analogous to that of 14a, starting from 13a. Purification was carried out by silica gel column chromatography (dichloromethane/methanol: 85/15). Yield: $91 \%$, m.p. $220-221{ }^{\circ} \mathrm{C}\left(\mathrm{EtOAc} / \mathrm{Et}_{2} \mathrm{O}\right) .{ }^{1} \mathrm{H}$ NMR $\left(600 \mathrm{MHz}\right.$, Acetone- $\left.d_{6}\right) \delta 10.80-$ 10.70 (brs, 1H, NH), 7.88 (d, $J=5.6 \mathrm{~Hz}, 1 \mathrm{H}, \mathrm{H}-5), 7.70-7.65$ (m, 3H, phenyl H-2,6, H2), 7.47-7.40 (m, 3H, phenyl H-3,5, H-4), 7.26 ( $\mathrm{t}, J=7.7 \mathrm{~Hz}, 1 \mathrm{H}$, phenyl H-4), 3.52-3.46 (m, 4H, methylpiperazine H-2,6), 2.64-2.60 (m, 4H, methylpiperazine H-3,5), 2.32 (s, 3H, methylpiperazine $\left.\mathrm{CH}_{3}\right) .{ }^{13} \mathrm{C} \mathrm{NMR}\left(600 \mathrm{MHz}\right.$, Acetone- $\left.d_{6}\right) \delta 150.27(\mathrm{C}-7), 137.95(\mathrm{C}-5)$, 136.39 (phenyl C-1), 132.33 (C-3a), 129.72 (phenyl C-3,5), 127.91 (phenyl C-2,6), 126.76 (phenyl C-4), 125.24 (C-7a), 125.08 (C-2), 118.36 (C-3), 109.71 (C-4), 55.98 (methylpiperazine C-3,5), 49.52 (methylpiperazine C-2,6), 46.44 (methylpiperazine $\mathrm{CH}_{3}$ ). HR-MS (ESI) $\mathrm{m} / z$ : Calcd for $\mathrm{C}_{18} \mathrm{H}_{19} \mathrm{~N}_{4}$ : [M-H] $]^{-}=291.1615$, found 291.1612. Anal. Calcd for $\mathrm{C}_{18} \mathrm{H}_{20} \mathrm{~N}_{4}: \mathrm{C}, 73.94$; H, 6.89; N, 19.17. Found: C, 74.03; H, 6.92; N, 19.02 .

3-(3-Fluorophenyl)-7-(4-methylpiperazin-1-yl)-1H-pyrrolo[2,3-c]pyridine (14f): This compound was synthesized using a procedure analogous to that of 14a, starting from 13b. Purification was carried out by silica gel column chromatography (dichloromethane/ methanol: 85/15). Yield: 93\%, m.p. 209-210 ${ }^{\circ} \mathrm{C}$ (EtOAc/n-pentane). ${ }^{1} \mathrm{H}$ NMR (600 MHz, Acetone- $\left.d_{6}\right) \delta 11.08-10.87$ (brs, $\left.1 \mathrm{H}, \mathrm{NH}\right), 7.90(\mathrm{~d}, J=5.7 \mathrm{~Hz}, 1 \mathrm{H}, \mathrm{H}-5), 7.77$ (s, 1H, H-2), 7.567.52 (m, 1H, fluorophenyl H-6), 7.50-7.40 (m, 3H, fluorophenyl H-5, H-4, fluorophenyl H-2), 7.05-6.98 (m, 1H, fluorophenyl H-4), 3.55-3.48 (m, 4H, methylpiperazine H-2,6), 2.72-2.65 (m, 4H, methylpiperazine $\mathrm{H}-3,5), 2.36\left(\mathrm{~s}, 3 \mathrm{H}\right.$, methylpiperazine $\left.\mathrm{CH}_{3}\right) .{ }^{13} \mathrm{C} \mathrm{NMR}(151 \mathrm{MHz}$, Acetone- $\left.d_{6}\right) \delta 165.07,163.46$ (fluorophenyl C-3), 150.10 (C-7), 138.92, 138.87 (fluorophenyl C-1), 138.21 (C-5), 132.12 (C-3a), 131.54, 131.48 (fluorophenyl C-5), 125.93 (C-7a), 125.33 (C2), 123.67 (fluorophenyl C-6), 117.06 (C-3), 114.20, 114.05 (fluorophenyl C-2), 113.28, 113.14 (fluorophenyl C-4), 109.62 (C-4), 55.65 (methylpiperazine C-3,5), 49.14 (methylpiperazine C-2,6), 46.02 (methylpiperazine $\mathrm{CH}_{3}$ ). HR-MS (ESI) $\mathrm{m} / z$ : Calcd for $\mathrm{C}_{18} \mathrm{H}_{20} \mathrm{FN}_{4}:[\mathrm{M}+\mathrm{H}]^{+}=$ 311.1667, found 311.1670. Anal. Calcd for $\mathrm{C}_{18} \mathrm{H}_{19} \mathrm{FN}_{4}$ : C, 69.66; H, 6.17; N, 18.05. Found: $\mathrm{C}$, 69.43; H, 6.14; N, 18.21.

4-Chloro-7-iodo-5-(4-methoxybenzyl)-5H-pyrrolo[3,2- $d$ ]pyrimidine (22): This compound was synthesized using a procedure analogous to that of $\mathbf{8}$, starting from 21 . Yield: 99\%, m.p. $180-181{ }^{\circ} \mathrm{C}\left(\mathrm{CH}_{2} \mathrm{Cl}_{2} / \mathrm{Et}_{2} \mathrm{O}\right) .{ }^{1} \mathrm{H} \mathrm{NMR}\left(600 \mathrm{MHz}, \mathrm{CDCl}_{3}\right) \delta 8.78(\mathrm{~s}, 1 \mathrm{H}, \mathrm{H}-2), 7.52$ (s, 1H, H-6), $7.08(\mathrm{~d}, J=8.6 \mathrm{~Hz}, 2 \mathrm{H}$, methoxybenzyl H-2,6), $6.88(\mathrm{~d}, J=8.6 \mathrm{~Hz}, 2 \mathrm{H}$, methoxybenzyl H-3,5), 5.64 (s, 2H, methoxybenzyl $\left.\mathrm{CH}_{2}\right), 3.79\left(\mathrm{~s}, 3 \mathrm{H}\right.$, methoxybenzyl $\left.\mathrm{CH}_{3} \mathrm{O}\right) .{ }^{13} \mathrm{C}$ NMR (151 MHz, CDCl$)_{3}$ ) 159.88 (methoxybenzyl C-4), 152.65 (C-7a), 150.64 (C-2), 142.62 (C-4), 140.09 (C-6), 128.76 (methoxybenzyl C-2,6), 127.81 (methoxybenzyl C-1), 124.45 (C-4a), 114.69 (methoxybenzyl C-3,5), $57.91(\mathrm{C}-7), 55.45\left(\mathrm{CH}_{3} \mathrm{O}\right), 52.29\left(\mathrm{CH}_{2}\right)$. HR-MS (ESI) $\mathrm{m} / z$ : Calcd for $\mathrm{C}_{14} \mathrm{H}_{12} \mathrm{ClIN}_{3} \mathrm{O}:[\mathrm{M}+\mathrm{H}]^{+}=399.9709$, found 399.9718 .

4-Chloro-5-(4-methoxybenzyl)-7-phenyl-5H-pyrrolo[3,2-d]pyrimidine (23a): This compound was synthesized using a procedure analogous to that of 9a, starting from 22. Purification was carried out by silica gel column chromatography (cyclohexane/ethyl acetate: 9/1). Yield: $94 \%$, m.p. $93-94{ }^{\circ} \mathrm{C}\left(\mathrm{CHCl}_{3} / n\right.$-hexane). ${ }^{1} \mathrm{H} \mathrm{NMR}\left(600 \mathrm{MHz}, \mathrm{CDCl}_{3}\right) \delta 8.80$ (s, 
1H, H-2), 8.00 (d, J = 7.4 Hz, 2H, phenyl H-2,6), $7.71(\mathrm{~s}, 1 \mathrm{H}, \mathrm{H}-6), 7.45(\mathrm{t}, J=7.7 \mathrm{~Hz}, 2 \mathrm{H}$, phenyl H-3,5), $7.31(\mathrm{t}, J=7.7 \mathrm{~Hz}, 1 \mathrm{H}$, phenyl $\mathrm{H}-4), 7.11(\mathrm{~d}, J=8.7 \mathrm{~Hz}, 2 \mathrm{H}$, methoxybenzyl $\mathrm{H}-2,6), 6.88\left(\mathrm{~d}, J=8.7 \mathrm{~Hz}, 2 \mathrm{H}\right.$, methoxybenzyl H-3,5), $5.70\left(\mathrm{~s}, 2 \mathrm{H}, \mathrm{CH}_{2}\right), 3.79\left(\mathrm{~s}, 3 \mathrm{H}, \mathrm{CH}_{3} \mathrm{O}\right)$. ${ }^{13} \mathrm{C}$ NMR (151 MHz, $\left.\mathrm{CDCl}_{3}\right) \delta 159.76$ (methoxybenzyl C-4), 150.17 (C-2), 149.91 (C-7a), 142.67 (C-4), 133.65 (C-6), 132.13 (phenyl C-1), 128.97 (phenyl C-3,5), 128.53 (methoxybenzyl C-2,6), 127.12 (phenyl C-4), 127.10 (phenyl C-2,6), 124.91 (C-4a), 117.21 (C-7), 114.75 (methoxybenzyl C-1), 114.65 (methoxybenzyl C-3,5), $55.47\left(\mathrm{CH}_{3} \mathrm{O}\right), 51.89\left(\mathrm{CH}_{2}\right)$. HR-MS (ESI) $m / z$ : Calcd for $\mathrm{C}_{20} \mathrm{H}_{17} \mathrm{ClN}_{3} \mathrm{O}:[\mathrm{M}+\mathrm{H}]^{+}=350.1055$, found 350.1065 .

4-Chloro-7-(3-fluorophenyl)-5-(4-methoxybenzyl)-5H-pyrrolo[3,2-d]pyrimidine (23b): This compound was synthesized using a procedure analogous to that of $\mathbf{9 a}$, starting from 22. Purification was carried out by silica gel column chromatography (cyclohexane/ethyl acetate: $8 / 2)$. Yield: $90 \%$, m.p. $88-89{ }^{\circ} \mathrm{C}\left(\mathrm{CHCl}_{3} / n\right.$-hexane). ${ }^{1} \mathrm{H}$ NMR $\left(600 \mathrm{MHz}, \mathrm{CDCl}_{3}\right) \delta$ $8.81(\mathrm{~s}, 1 \mathrm{H}, \mathrm{H}-2), 7.82-7.79(\mathrm{~m}, 1 \mathrm{H}$ fluorophenyl H-2), 7.79-7.76 $(\mathrm{m}, 1 \mathrm{H}$, fluorophenyl $\mathrm{H}-6)$, $7.71(\mathrm{~s}, 1 \mathrm{H}, \mathrm{H}-6), 7.40-7.37(\mathrm{~m}, 1 \mathrm{H}$, fluorophenyl H-5), $7.12(\mathrm{~d}, J=8.5 \mathrm{~Hz}, 2 \mathrm{H}$, methoxybenzyl $\mathrm{H}-2,6), 7.00-6.96$ ( $\mathrm{m}, 1 \mathrm{H}$, fluorophenyl H-4), 6.89 (d, $J=8.6 \mathrm{~Hz}, 2 \mathrm{H}$, methoxybenzyl H-3,5), $5.70\left(\mathrm{~s}, 2 \mathrm{H}, \mathrm{CH}_{2}\right), 3.80\left(\mathrm{~s}, 3 \mathrm{H}, \mathrm{CH}_{3} \mathrm{O}\right) .{ }^{13} \mathrm{C}$ NMR $\left(151 \mathrm{MHz}, \mathrm{CDCl}_{3}\right) \delta 164.10,162.48$ (fluorophenyl C-3), 159.81 (methoxybenzyl C-4), 150.28 (C-2), 149.73 (C-7a), 142.80 (C-4), 134.28, 134.23 (fluorophenyl C-1) 133.77 (C-6), 130.37, 130.31 (fluorophenyl C-5), 128.59 (methoxybenzyl C-2,6), 124.96 (C-4a), 122.44 (fluorophenyl C-6), 115.92 (C-7), 114.75 (methoxybenzyl C-1), 114.69 (methoxybenzyl C-3,5), 113.94, 113.87 (fluorophenyl C-2), 113.79, 113.73 (fluorophenyl C-4), $55.46\left(\mathrm{CH}_{3} \mathrm{O}\right), 51.96\left(\mathrm{CH}_{2}\right)$. HR-MS (ESI) $\mathrm{m} / \mathrm{z}$ : Calcd for $\mathrm{C}_{20} \mathrm{H}_{16} \mathrm{ClFN}_{3} \mathrm{O}$ : [M $+\mathrm{H}]^{+}=368.0961$, found 368.0970 .

5-(4-Methoxybenzyl)-N,7-diphenyl-5H-pyrrolo[3,2- $d$ ]pyrimidin-4-amine (24a): This compound was synthesized using a procedure analogous to that of $\mathbf{1 0 a}$, starting from 23a. Purification was carried out by silica gel column chromatography (cyclohexane/ethyl acetate: $7 / 3)$. Yield: $87 \%$, m.p. $57-58{ }^{\circ} \mathrm{C}\left(\mathrm{CH}_{2} \mathrm{Cl}_{2} / \mathrm{Et}_{2} \mathrm{O}\right) .{ }^{1} \mathrm{H}$ NMR $\left(600 \mathrm{MHz}\right.$, Acetone- $\left.d_{6}\right) \delta$ 8.44 (s, 1H, H-2), 8.28 (d, J = 7.6 Hz, 2H, 7-phenyl H-2,6), $8.16(\mathrm{~s}, 1 \mathrm{H}, \mathrm{H}-6), 7.49(\mathrm{~d}, J=7.9 \mathrm{~Hz}$, 2H, $\mathrm{N}^{4}$-phenyl H-2,6), 7.41 (t, J = 7.7 Hz, 2H, 7-phenyl H-3,5), 7.33 (brs, 1H, NH), 7.30-7.19 (m, 5H, $\mathrm{N}^{4}$-phenyl H-3,5, methoxybenzyl H-2,6, 7-phenyl H-4), 7.06-6.97 (m, 3H, $\mathrm{N}^{4}$-phenyl $\mathrm{H}-4$, methoxybenzyl H-3,5), $5.81\left(\mathrm{~s}, 2 \mathrm{H}, \mathrm{CH}_{2}\right), 3.77\left(\mathrm{~s}, 3 \mathrm{H}, \mathrm{CH}_{3} \mathrm{O}\right) .{ }^{13} \mathrm{C} \mathrm{NMR}(151 \mathrm{MHz}$, Acetone- $d_{6}$ ) $\delta 160.91$ (methoxybenzyl C-4), 151.09 (C-2), 149.36 (C-4), 148.44 (C-7a), 140.77 ( $\mathrm{N}^{4}$-phenyl C-1), 134.92 (7-phenyl C-1), 132.90 (C-6), 130.46 (methoxybenzyl C-1), 129.43 (7-phenyl C-3,5), 129.28 ( $\mathrm{N}^{4}$-phenyl C-3,5), 128.81 (methoxybenzyl C-2,6), 127.39 (7-phenyl C-2,6), 126.74 (7-phenyl C-4), $123.61\left(\mathrm{~N}^{4}\right.$-phenyl C-4), $121.63\left(\mathrm{~N}^{4}\right.$-phenyl C-2,6), 117.03 (C-4a), 116.11 (C-7), 115.71 (methoxybenzyl C-3,5), $55.75\left(\mathrm{CH}_{3} \mathrm{O}\right), 53.24\left(\mathrm{CH}_{2}\right)$. HR-MS (ESI) $m / z$ : Calcd for $\mathrm{C}_{26} \mathrm{H}_{23} \mathrm{~N}_{4} \mathrm{O}:[\mathrm{M}+\mathrm{H}]^{+}=407.1867$, found 407.1864. Anal. Calcd for $\mathrm{C}_{26} \mathrm{H}_{22} \mathrm{~N}_{4} \mathrm{O}: \mathrm{C}, 76.83 ; \mathrm{H}, 5.46 ; \mathrm{N}, 13.78$. Found: $\mathrm{C}, 76.99 ; \mathrm{H}, 5.45 ; \mathrm{N}, 13.60$.

7-(3-Fluorophenyl)-5-(4-methoxybenzyl)- $\mathrm{N}$-phenyl-5H-pyrrolo[3,2-d]pyrimidin-4-amine (24b): This compound was synthesized using a procedure analogous to that of $10 \mathbf{a}$, starting from 23b. Purification was carried out by silica gel column chromatography (cyclohexane/ethyl acetate: 8/2). Yield: $85 \%$, m.p. $131-132{ }^{\circ} \mathrm{C}\left(\mathrm{CH}_{2} \mathrm{Cl}_{2} / \mathrm{Et}_{2} \mathrm{O}\right) .{ }^{1} \mathrm{H} \mathrm{NMR}(600 \mathrm{MHz}$, Acetone- $\left.d_{6}\right) \delta 8.46(\mathrm{~s}, 1 \mathrm{H}, \mathrm{H}-2), 8.26-8.22(\mathrm{~m}, 2 \mathrm{H}$, fluorophenyl H-2, H-6), 8.06-8.02 (m, $1 \mathrm{H}$, fluorophenyl H-6), $7.48(\mathrm{~d}, J=7.9 \mathrm{~Hz}, 2 \mathrm{H}$, phenyl H-2,6), 7.46-7.42 (m, 1H, fluorophenyl H-5), 7.33 (brs, $1 \mathrm{H}, \mathrm{NH}), 7.31-7.24(\mathrm{~m}, 4 \mathrm{H}$, phenyl H-3,5, methoxybenzyl H-2,6), 7.05-6.96 $(\mathrm{m}, 4 \mathrm{H}$, phenyl $\mathrm{H}-4$, methoxybenzyl H-3,5, fluorophenyl $\mathrm{H}-4), 5.80\left(\mathrm{~s}, 2 \mathrm{H}, \mathrm{CH}_{2}\right), 3.77\left(\mathrm{~s}, 3 \mathrm{H}, \mathrm{CH}_{3} \mathrm{O}\right) .{ }^{13} \mathrm{C} \mathrm{NMR}$ (151 MHz, Acetone- $d_{6}$ ) $\delta 164.92,163.32$ (fluorophenyl C-3), 160.95 (methoxybenzyl C-4), 151.30 (C-2), 149.25 (C-4), 148.51 (C-7a), 140.63 (phenyl C-1), 137.44, 137.38 (fluorophenyl C-1), 133.43 (C-6), 131.01, 130.95 (fluorophenyl C-5), 130.26 (methoxybenzyl C-1), 129.44 (phenyl C-3,5), 128.84 (methoxybenzyl C-2,6), 123.74 (phenyl C-4), 122.79 (fluorophenyl C-6), 121.74 (phenyl C-2,6), 117.06 (C-4a), 115.74 (methoxybenzyl C-3,5), 114.69 (C-7), 113.93, 113.78 (fluorophenyl C-2), 113.14, 113.00 (fluorophenyl C-4), $55.75\left(\mathrm{CH}_{3} \mathrm{O}\right), 53.34\left(\mathrm{CH}_{2}\right)$. HR-MS (ESI) $\mathrm{m} / \mathrm{z}$ : Calcd for $\mathrm{C}_{26} \mathrm{H}_{22} \mathrm{FN}_{4} \mathrm{O}:[\mathrm{M}+\mathrm{H}]^{+}=425.1773$, found 425.1767. Anal. Calcd for $\mathrm{C}_{26} \mathrm{H}_{21} \mathrm{FN}_{4} \mathrm{O}: \mathrm{C}$, 73.57; H, 4.99; N, 13.20. Found: C, 73.73; H, 5.08; N, 13.02 . 
5-(4-Methoxybenzyl)-N-(3,4,5-trimethoxyphenyl)-7-phenyl-5H-pyrrolo[3,2- $d$ ]pyrimidin4-amine (24c): This compound was synthesized using a procedure analogous to that of 10a, starting from 23a. Purification was carried out by silica gel column chromatography (cyclohexane/ethyl acetate: $7 / 3)$. Yield: $60 \%$, m.p. $187-188^{\circ} \mathrm{C}\left(\mathrm{CH}_{2} \mathrm{Cl}_{2} / \mathrm{Et}_{2} \mathrm{O}\right) \cdot{ }^{1} \mathrm{H} \mathrm{NMR}(600 \mathrm{MHz}$, Acetone- $\left.d_{6}\right) \delta 8.45(\mathrm{~s}, 1 \mathrm{H}, \mathrm{H}-2), 8.28(\mathrm{~d}, J=8.1 \mathrm{~Hz}, 2 \mathrm{H}$, phenyl H-2,6), $8.15(\mathrm{~s}, 1 \mathrm{H}, \mathrm{H}-6), 7.41$ $(\mathrm{t}, J=7.8 \mathrm{~Hz}, 2 \mathrm{H}$, phenyl H-3,5), $7.31(\mathrm{~d}, J=8.7 \mathrm{~Hz}, 2 \mathrm{H}$, methoxybenzyl H-2,6), $7.23(\mathrm{t}, J=$ $7.4 \mathrm{~Hz}, 1 \mathrm{H}$, phenyl H-4), 7.12 (brs, 1H, NH), 7.05 (d, J = 8.7 Hz, 2H, methoxybenzyl H-3,5), $6.78(\mathrm{~s}, 2 \mathrm{H}$, trimethoxyphenyl $\mathrm{H}-2,6), 5.80\left(\mathrm{~s}, 2 \mathrm{H}, \mathrm{CH}_{2}\right), 3.79\left(\mathrm{~s}, 3 \mathrm{H}\right.$, methoxybenzyl $\left.\mathrm{CH}_{3} \mathrm{O}\right)$, $3.78\left(\mathrm{~s}, 6 \mathrm{H}\right.$, trimethoxyphenyl $\left.\mathrm{CH}_{3} \mathrm{O}-3,5\right), 3.68\left(\mathrm{~s}, 3 \mathrm{H}\right.$, trimethoxyphenyl $\left.\mathrm{CH}_{3} \mathrm{O}-4\right) .{ }^{13} \mathrm{C}$ NMR $\left(151 \mathrm{MHz}\right.$, Acetone- $\left.d_{6}\right) \delta 161.00$ (methoxybenzyl C-4), 154.22 (trimethoxyphenyl C-3,5), 151.16 (C-2), 149.17 (C-4), 148.48 (C-7a), 136.56 (trimethoxyphenyl C-1), 135.08 (trimethoxyphenyl C-4), 134.93 (phenyl C-1), 132.94 (C-6), 130.45 (methoxybenzyl C-1), 129.28 (phenyl C-3,5), 128.84 (methoxybenzyl C-2,6), 127.39 (phenyl C-2,6), 126.74 (phenyl C-4), 116.91 (C-4a), 116.07 (C-7), 115.83 (methoxybenzyl C-3,5), 99.69 (trimethoxyphenyl C-2,6), 60.71 (trimethoxyphenyl $\left.\mathrm{CH}_{3} \mathrm{O}-4\right), 56.51$ (trimethoxyphenyl $\left.\mathrm{CH}_{3} \mathrm{O}-3,5\right), 55.77$ (methoxybenzyl $\mathrm{CH}_{3} \mathrm{O}$ ), $53.29\left(\mathrm{CH}_{2}\right.$ ). HR-MS (ESI) $m / z$ : Calcd for $\mathrm{C}_{29} \mathrm{H}_{29} \mathrm{~N}_{4} \mathrm{O}_{4}:[\mathrm{M}+\mathrm{H}]^{+}=497.2184$, found 497.2165. Anal. Calcd for $\mathrm{C}_{29} \mathrm{H}_{28} \mathrm{~N}_{4} \mathrm{O}_{4}$ : C, 70.15; H, 5.68; N, 11.28. Found: C, 69.99; H, 5.51; N, 11.44 .

7-(3-Fluorophenyl)-5-(4-methoxybenzyl)- $N$-(3,4,5-trimethoxyphenyl)-5H-pyrrolo[3,2d]pyrimidin-4-amine (24d): This compound was synthesized using a procedure analogous to that of 10a, starting from $\mathbf{2 3 b}$. Purification was carried out by silica gel column chromatography (cyclohexane/ethyl acetate: 6/4). Yield: $63 \%$, m.p. $96-97{ }^{\circ} \mathrm{C}\left(\mathrm{CH}_{2} \mathrm{Cl}_{2} / n\right.$-pentane). ${ }^{1} \mathrm{H}$ NMR $\left(600 \mathrm{MHz}\right.$, Acetone- $\left.d_{6}\right) \delta 8.47$ (s, 1H, H-2), 8.26-8.20 (m, 2H, H-6, fluorophenyl H-2), 8.07-8.03 (m, 1H, fluorophenyl H-6), 7.47-7.40 (m, 1H, fluorophenyl H-5), 7.30 (d, J = 8.7 $\mathrm{Hz}, 2 \mathrm{H}$, methoxybenzyl H-2,6), 7.15 (brs, 1H, NH), 7.05 (d, J = 8.7 Hz, 2H, methoxybenzyl $\mathrm{H}-3,5)$, 7.02-6.97 (m, 1H, fluorophenyl H-4), 6.77 (s, 2H, trimethoxyphenyl H-2,6), 5.81 (s, $\left.2 \mathrm{H}, \mathrm{CH}_{2}\right), 3.79\left(\mathrm{~s}, 3 \mathrm{H}\right.$, methoxybenzyl $\left.\mathrm{CH}_{3} \mathrm{O}\right), 3.78\left(\mathrm{~s}, 6 \mathrm{H}\right.$, trimethoxyphenyl $\left.\mathrm{CH}_{3} \mathrm{O}-3,5\right)$, 3.67 (s, 3H, trimethoxyphenyl $\left.\mathrm{CH}_{3} \mathrm{O}-4\right) .{ }^{13} \mathrm{C}$ NMR $\left(151 \mathrm{MHz}\right.$, Acetone- $\left.d_{6}\right) \delta 164.92,163.32$ (fluorophenyl C-3), 161.05 (methoxybenzyl C-4), 154.23 (trimethoxyphenyl C-3,5), 151.39 (C2), 149.07 (C-4), 148.53 (C-7a), 137.46, 137.40 (fluorophenyl C-1), 136.41 (trimethoxyphenyl C-1), 135.17 (trimethoxyphenyl C-4), 133.47 (C-6), 131.02, 130.96 (fluorophenyl C-5), 130.24 (methoxybenzyl C-1), 128.86 (methoxybenzyl C-2,6), 122.78 (fluorophenyl C-6), 116.94 (C-4a), 115.87 (methoxybenzyl C-3,5), 114.65 (C-7), 11393, 113.78 (fluorophenyl C-2), 113.15, 113.01 (fluorophenyl C-4), 99.78 (trimethoxyphenyl C-2,6), 60.71 (trimethoxyphenyl $\mathrm{CH}_{3} \mathrm{O}$ 4), 56.51 (trimethoxyphenyl $\left.\mathrm{CH}_{3} \mathrm{O}-3,5\right), 55.78$ (methoxybenzyl $\mathrm{CH}_{3} \mathrm{O}$ ), $53.39\left(\mathrm{CH}_{2}\right)$. HR-MS (ESI) $m / z$ : Calcd for $\mathrm{C}_{29} \mathrm{H}_{28} \mathrm{FN}_{4} \mathrm{O}_{4}:[\mathrm{M}+\mathrm{H}]^{+}=515.2090$, found 515.2075. Anal. Calcd for $\mathrm{C}_{29} \mathrm{H}_{27} \mathrm{FN}_{4} \mathrm{O}_{4}$ : C, 67.69; H, 5.29; N, 10.89. Found: C, 67.48; H, 5.16; N, 11.03.

5-(4-Methoxybenzyl)-4-(4-methylpiperazin-1-yl)-7-phenyl-5H-pyrrolo[3,2-d]pyrimidine (25a): To a solution of $\mathbf{2 3 a}$ ( $30 \mathrm{mg}, 0.09 \mathrm{mmol}$ ) in dimethylsulfoxide (1 mL), 1-methylpiperazine $(0.1 \mathrm{~mL}, 0.90 \mathrm{mmol})$ was added, and the solution was heated at $120{ }^{\circ} \mathrm{C}$ for $20 \mathrm{~h}$. Then, the mixture was diluted with water and extracted with ethyl acetate. The organic phase was dried $\left(\mathrm{Na}_{2} \mathrm{SO}_{4}\right)$ and concentrated to dryness, and the residue was purified by silica gel column chromatography (ethyl acetate/methanol: 8/2) to give 25a in almost quantitative yield. M.p. 150-151 ${ }^{\circ} \mathrm{C}\left(\mathrm{CH}_{2} \mathrm{Cl}_{2} / n\right.$-pentane). ${ }^{1} \mathrm{H}$ NMR (600 MHz, Acetone- $\left.d_{6}\right) \delta 8.57(\mathrm{~s}, 1 \mathrm{H}, \mathrm{H}-2), 8.22(\mathrm{~d}$, $J=7.6 \mathrm{~Hz}, 2 \mathrm{H}$, phenyl H-2,6), $8.11(\mathrm{~s}, 1 \mathrm{H}, \mathrm{H}-6), 7.37$ ( $\mathrm{t}, J=7.7 \mathrm{~Hz}, 2 \mathrm{H}$, phenyl H-3,5), 7.23-7.19 (m, 3H, phenyl H-4, methoxybenzyl H-2,6), $6.82(\mathrm{~d}, J=8.6 \mathrm{~Hz}, 2 \mathrm{H}$, methoxybenzyl H-3,5), 5.52 (s, 2H, $\mathrm{CH}_{2}$ ), $3.71\left(\mathrm{~s}, 3 \mathrm{H}, \mathrm{CH}_{3} \mathrm{O}\right.$ ), 3.46-3.37 (brs, 4H, methylpiperazine $\left.\mathrm{H}-2,6\right), 2.68-2.60$ (brs, $4 \mathrm{H}$, methylpiperazine $\mathrm{H}-3,5), 2.32$ (s, 3H, methylpiperazine $\left.\mathrm{CH}_{3}\right) .{ }^{13} \mathrm{C}$ NMR $(151 \mathrm{MHz}$, Acetone$d_{6}$ ) $\delta 160.31$ (methoxybenzyl C-4), 156.02 (C-4), 151.09 (C-2), 150.79 (C-7a), 134.67 (phenyl C-1), 132.92 (C-6), 131.00 (methoxybenzyl C-1), 129.67 (phenyl C-3,5), 129.25 (methoxybenzyl C-2,6), 127.29 (phenyl C-2,6), 126.86 (phenyl C-4), 120.77 (C-4a), 117.66 (C-7), 114.90 (methoxybenzyl $\mathrm{C}-3,5), 55.53\left(\mathrm{CH}_{3} \mathrm{O}\right), 55.46$ (methylpiperazine $\left.\mathrm{C}-3,5\right), 51.63$ (methylpiperazine $\left.\mathrm{C}-2,6\right), 50.90$ $\left(\mathrm{CH}_{2}\right), 46.42$ (methylpiperazine $\mathrm{CH}_{3}$ ). HR-MS (ESI) $m / z$ : Calcd for $\mathrm{C}_{25} \mathrm{H}_{28} \mathrm{~N}_{5} \mathrm{O}:[\mathrm{M}+\mathrm{H}]^{+}=$ 414.2289, found 414.2286. Anal. Calcd for $\mathrm{C}_{25} \mathrm{H}_{27} \mathrm{~N}_{5} \mathrm{O}$ : C, 72.61; H, 6.58; N, 16.94. Found: $\mathrm{C}$, $72.90 ; \mathrm{H}, 6.69 ; \mathrm{N}, 16.63$. 
7-(3-Fluorophenyl)-5-(4-methoxybenzyl)-4-(4-methylpiperazin-1-yl)-5H-pyrrolo[3,2-d] pyrimidine $(\mathbf{2 5 b})$ : This compound was synthesized using a procedure analogous to that of 25a, starting from 23b. Purification was carried out by silica gel column chromatography (dichloromethane/methanol: 9/1). Yield: 97\%, m.p. 151-152 ${ }^{\circ} \mathrm{C}\left(\mathrm{CH}_{2} \mathrm{Cl}_{2} / n\right.$-pentane). ${ }^{1} \mathrm{H}$ NMR $\left(600 \mathrm{MHz}\right.$, Acetone- $\left.d_{6}\right) \delta 8.58$ (s, 1H, H-2), 8.20 (s, 1H, H-6), 8.18-8.14 (m, 1H, fluorophenyl H-2), 8.02-7.97 (m, 1H, fluorophenyl H-6), 7.42-7.35 (m, 1H, fluorophenyl H-5), $7.20(\mathrm{~d}, J=8.6 \mathrm{~Hz}, 2 \mathrm{H}$, methoxybenzyl H-2,6), 6.98-6.93 (m, 1H, fluorophenyl H-4), 6.82 $\left(\mathrm{d}, J=8.6 \mathrm{~Hz}, 2 \mathrm{H}\right.$, methoxybenzyl H-3,5), $5.53\left(\mathrm{~s}, 2 \mathrm{H}, \mathrm{CH}_{2}\right), 3.71\left(\mathrm{~s}, 3 \mathrm{H}, \mathrm{CH}_{3} \mathrm{O}\right), 3.50-3.38$ (brs, 4H, methylpiperazine H-2,6), 2.67-2.58 (brs, 4H, methylpiperazine, H-3,5), 2.30 (s, 3H, methylpiperazine $\left.\mathrm{CH}_{3}\right) .{ }^{13} \mathrm{C}$ NMR $\left(151 \mathrm{MHz}\right.$, Acetone- $\left.d_{6}\right) \delta 164.86,163.26$ (fluorophenyl C-3), 160.38 (methoxybenzyl C-4), 156.14 (C-4), 151.28 (C-2), 150.65 (C-7a), 137.18, 137.12 (fluorophenyl C-1), 133.49 (C-6), 131.01, 130.95 (fluorophenyl C-5), 130.85 (methoxybenzyl C-1), 129.69 (methoxybenzyl C-2,6), 122.77 (fluorophenyl C-6), 121.89 (C-7), 116.27 (C-4a), 114.96 (methoxybenzyl C-3,5), 113.84, 113.69 (fluorophenyl C-2), 113.29, 113.15 (fluorophenyl C-4), $55.55\left(\mathrm{CH}_{3} \mathrm{O}\right.$, methylpiperazine $\left.\mathrm{C}-3,5\right), 51.79\left(\mathrm{CH}_{2}\right), 50.96$ (methylpiperazine $\mathrm{C}-2,6), 46.46$ (methylpiperazine $\mathrm{CH}_{3}$ ). HR-MS (ESI) $\mathrm{m} / z$ : Calcd for $\mathrm{C}_{25} \mathrm{H}_{27} \mathrm{FN}_{5} \mathrm{O}:[\mathrm{M}+\mathrm{H}]^{+}$ $=432.2195$, found 432.2181. Anal. Calcd for $\mathrm{C}_{25} \mathrm{H}_{26} \mathrm{FN}_{5} \mathrm{O}: \mathrm{C}, 69.59 ; \mathrm{H}, 6.07 ; \mathrm{N}, 16.23$. Found: C, 69.80; H, 6.11; N, 16.00 .

N,7-Diphenyl-5H-pyrrolo[3,2-d]pyrimidin-4-amine (26a): This compound was synthesized using a procedure analogous to that of 14a, starting from 24a. Purification was carried out by silica gel column chromatography (dichloromethane/ethyl acetate: 7/3). Yield: 99\%, m.p. $>250{ }^{\circ} \mathrm{C}$ (EtOAc). ${ }^{1} \mathrm{H}$ NMR (600 MHz, Acetone- $\left.d_{6}\right) \delta 10.76-10.67$ (brs, $1 \mathrm{H}$, pyrrole $\mathrm{NH}), 8.64-8.58$ (brs, $1 \mathrm{H}$, pyrimidinamine $\mathrm{NH}), 8.54(\mathrm{~s}, 1 \mathrm{H}, \mathrm{H}-2), 8.26(\mathrm{dd}, J=8.2,1.1 \mathrm{~Hz}, 2 \mathrm{H}$, 7-phenyl H-2,6), 8.02 (s, 1H, H-6), 7.93 (dd, J = 7.7, $1.0 \mathrm{~Hz}, 2 \mathrm{H}, \mathrm{N}$-phenyl H-2,6), 7.39 (t, $J=$ $7.8 \mathrm{~Hz}, 2 \mathrm{H}, 7$-phenyl H-3,5), $7.35(\mathrm{t}, J=7.5 \mathrm{~Hz}, 2 \mathrm{H}, \mathrm{N}$-phenyl H-3,5), $7.21(\mathrm{t}, J=7.4 \mathrm{~Hz}, 1 \mathrm{H}$, 7-phenyl H-4), 7.05 ( $\mathrm{t}, J=7.3 \mathrm{~Hz}, 1 \mathrm{H}, \mathrm{N}$-phenyl H-4). ${ }^{13} \mathrm{C}$ NMR (151 MHz, Acetone- $\left.d_{6}\right) \delta$ 151.09 (C-2), 148.19 (C-4), 147.05 (C-7a), 141.33 (N-phenyl C-1), 135.34 (7-phenyl C-1), 129.64 (7-phenyl C-3,5), 129.24 (N-phenyl C-3,5), 127.27 (7-phenyl C-2,6), 126.63 (7-phenyl C-4), 126.42 (C-6), 123.32 (N-phenyl C-4), 120.81 (N-phenyl C-2,6), 117.10 (C-4a), 116.53 (C-7). HR-MS (ESI) $m / z$ : Calcd for $\mathrm{C}_{18} \mathrm{H}_{15} \mathrm{~N}_{4}$ : $[\mathrm{M}+\mathrm{H}]^{+}=287.1292$, found 287.1296. Anal. Calcd for $\mathrm{C}_{18} \mathrm{H}_{14} \mathrm{~N}_{4}$ : C, 75.50; H, 4.93; N, 19.57. Found: C, 75.36; H, 4.90; N, 19.69.

7-(3-Fluorophenyl)- $N$-phenyl-5H-pyrrolo[3,2-d]pyrimidin-4-amine (26b): This compound was synthesized using a procedure analogous to that of $14 a$, starting from $24 \mathbf{b}$. Purification was carried out by silica gel column chromatography (dichloromethane/ethyl acetate: $75 / 25)$. Yield: $96 \%$ m.p. $>250{ }^{\circ} \mathrm{C}$ (EtOAc). ${ }^{1} \mathrm{H}$ NMR $\left(600 \mathrm{MHz}\right.$, Acetone- $\left.d_{6}\right) \delta$ 10.87-10.71 (brs, 1H, pyrrole NH), 8.63-8.58 (brs, 1H, pyrimidinamine NH), 8.55 (s, 1H, H-2), 8.24-8.20 (m, 1H, fluorophenyl H-2), 8.12 (s, 1H, H-6), 8.06-8.02 (m, 1H, fluorophenyl H-6), $7.93(\mathrm{~d}, J=7.7 \mathrm{~Hz}, 2 \mathrm{H}$, phenyl H-2,6), 7.39-7.36 (m, 1H, fluorophenyl H-5), $7.34(\mathrm{t}, J=7.5$ $\mathrm{Hz}, 2 \mathrm{H}$, phenyl H-3,5), $7.06(\mathrm{t}, J=7.3 \mathrm{~Hz}, 1 \mathrm{H}$, phenyl H-4), 6.99-6.93 (m 1H, fluorophenyl H-4). ${ }^{13} \mathrm{C}$ NMR (151 MHz, Acetone- $\left.d_{6}\right) \delta$ 164.93, 163.33 (fluorophenyl C-3), 151.35 (C-2), 148.25 (C-4), 147.07 (C-7a), 141.23 (phenyl C-2,6), 137.90, 137.84 (fluorophenyl C-1), 130.97, 130.91 (fluorophenyl C-5), 129.67 (phenyl C-3,5), 127.06 (C-6), 123.44 (phenyl C-4), 122.69 (fluorophenyl C-6), 120.88 (phenyl C-2,6), 116.58 (C-4a), 115.74 (C-7), 113.79, 113.64 (fluorophenyl C-2), 113.03, 112.89 (fluorophenyl C-4). HR-MS (ESI) $m / z$ : Calcd for $\mathrm{C}_{18} \mathrm{H}_{14} \mathrm{FN}_{4}$ : $[\mathrm{M}+\mathrm{H}]^{+}=305.1197$, found 305.1192. Anal. Calcd for $\mathrm{C}_{18} \mathrm{H}_{13} \mathrm{FN}_{4}$ : C, 71.04; $\mathrm{H}, 4.31 ; \mathrm{N}, 18.41$. Found: C, 70.79; H, 4.22; N, 18.60 .

$N$-(3,4,5-Trimethoxyphenyl)-7-phenyl-5H-pyrrolo[3,2- $d$ ]pyrimidin-4-amine (26c): This compound was synthesized using a procedure analogous to that of $\mathbf{1 4 a}$, starting from 24c. Purification was carried out by silica gel column chromatography (cyclohexane/ethyl acetate: 3/7). Yield: 97\%, m.p. > $250{ }^{\circ} \mathrm{C}$ (EtOAc). ${ }^{1} \mathrm{H}$ NMR (600 MHz, Acetone- $\left.d_{6}\right) \delta$ 10.85-10.79 (brs, $1 \mathrm{H}$, pyrrole $\mathrm{NH}), 8.70-8.63$ (brs, $1 \mathrm{H}$, pyrimidinamine $\mathrm{NH}), 8.53$ (s, $1 \mathrm{H}$, $\mathrm{H}-2), 8.26$ (d, J = 7.9 Hz, 2H, phenyl H-2,6), 8.01 (s, 1H, H-6), 7.39 (t, J = 7.5 Hz, 2H, phenyl $\mathrm{H}-3,5), 7.31$ (s, 2H, trimethoxyphenyl H-2,6), $7.21(\mathrm{t}, J=7.2 \mathrm{~Hz}, 1 \mathrm{H}$, phenyl H-4), 3.84 (s, $6 \mathrm{H}$, trimethoxyphenyl $\left.\mathrm{CH}_{3} \mathrm{O}-3,5\right), 3.71$ (s, 3H, $\left.\mathrm{CH}_{3} \mathrm{O}-4\right) .{ }^{13} \mathrm{C}$ NMR (151 MHz, Acetone- 
$\left.d_{6}\right) \delta 154.46$ (trimethoxyphenyl C-3,5), 151.17 (C-2), 148.32 (C-4), 147.07 (C-7a), 137.37 (trimethoxyphenyl C-1), 135.43 (phenyl C-1), 134.98 (trimethoxyphenyl C-4), 129.24 (phenyl C-3,5), 127.27 (phenyl C-2,6), 126.59 (phenyl C-4), 126.29 (C-6), 117.05 (C-4a), 116.48 (C-7), 99.14 (trimethoxyphenyl C-2,6), 60.75 (trimethoxyphenyl $\mathrm{CH}_{3} \mathrm{O}-4$ ), 56.52 (trimethoxyphenyl $\mathrm{CH}_{3} \mathrm{O}-3,5$ ). HR-MS (ESI) $\mathrm{m} / \mathrm{z}$ : Calcd for $\mathrm{C}_{21} \mathrm{H}_{19} \mathrm{~N}_{4} \mathrm{O}_{3}$ : $[\mathrm{M}-\mathrm{H}]^{-}=375.1462$, found 375.1456 . Anal. Calcd for $\mathrm{C}_{21} \mathrm{H}_{20} \mathrm{~N}_{4} \mathrm{O}_{3}$ : C, 67.01; $\mathrm{H}, 5.36 ; \mathrm{N}, 14.88$. Found: $\mathrm{C}, 66.84 ; \mathrm{H}, 5.29 ; \mathrm{N}, 15.09$. 7-(3-Fluorophenyl)- $\mathrm{N}$-(3,4,5-trimethoxyphenyl)-5H-pyrrolo[3,2-d]pyrimidin-4-amine (26d): This compound was synthesized using a procedure analogous to that of $\mathbf{1 4 a}$, starting from 24d. Purification was carried out by silica gel column chromatography (chloroform/ethyl acetate: 4/6). Yield: $93 \%$, m.p. $139-140{ }^{\circ} \mathrm{C}\left(\mathrm{EtOAc} / \mathrm{Et}_{2} \mathrm{O}\right) .{ }^{1} \mathrm{H} \mathrm{NMR}(600 \mathrm{MHz}$, Acetone- $\left.d_{6}\right) \delta 10.82-10.66$ (brs, $1 \mathrm{H}$, pyrrole $\left.\mathrm{NH}\right), 8.58-8.54(\mathrm{~m}, 2 \mathrm{H}$, pyrimidinamine $\mathrm{NH}$, $\mathrm{H}-2), 8.25-8.19(\mathrm{~m}, 1 \mathrm{H}$, fluorophenyl H-2), $8.11(\mathrm{~s}, 1 \mathrm{H}, \mathrm{H}-6), 8.06-8.02(\mathrm{~m}, 1 \mathrm{H}$, fluorophenyl H-6), 7.44-7.38 (m, 1H, fluorophenyl H-5), $7.28(\mathrm{~s}, 2 \mathrm{H}$, trimethoxyphenyl H-2,6), 7.00-6.93 (m, $1 \mathrm{H}$, fluorophenyl $\mathrm{H}-4), 3.84\left(\mathrm{~s}, 6 \mathrm{H}\right.$, trimethoxyphenyl $\left.\mathrm{CH}_{3} \mathrm{O}-3,5\right), 3.71\left(\mathrm{~s}, 3 \mathrm{H}, \mathrm{CH}_{3} \mathrm{O}-4\right) .{ }^{13} \mathrm{C}$ NMR (151 MHz, Acetone- $d_{6}$ ) $\delta 164.92,163.32$ (fluorophenyl C-3), 154.46 (trimethoxyphenyl C-3,5), 151.38 (C-2), 148.34 (C-4), 146.94 (C-7a), 137.91, 137.85 (fluorophenyl C-1), 137.16 (trimethoxyphenyl C-1), 135.07 (trimethoxyphenyl C-4), 130.97, 130.91 (fluorophenyl C-5), 126.97 (C-6), 122.68 (fluorophenyl C-6), 116.48 (C-4a), 115.69 (C-7), 113.77, 113.62 (fluorophenyl C-2), 113.02, 112.87 (fluorophenyl C-4), 99.19 (trimethoxyphenyl C-2,6), 60.75 (trimethoxyphenyl $\mathrm{CH}_{3} \mathrm{O}-4$ ), 56.52 (trimethoxyphenyl $\mathrm{CH}_{3} \mathrm{O}-3,5$ ). HR-MS (ESI) $\mathrm{m} / \mathrm{z}$ : Calcd for $\mathrm{C}_{21} \mathrm{H}_{18} \mathrm{FN}_{4} \mathrm{O}_{3}:[\mathrm{M}-\mathrm{H}]^{-}=393.1368$, found 393.1370. Anal. Calcd for $\mathrm{C}_{21} \mathrm{H}_{19} \mathrm{FN}_{4} \mathrm{O}_{3}: \mathrm{C}$, 63.95; H, 4.86; N, 14.21. Found: C, 64.12; H, 4.89; N, 14.05 .

4-(4-Methylpiperazin-1-yl)-7-phenyl-5H-pyrrolo[3,2- $d$ ]pyrimidine (26e): This compound was synthesized using a procedure analogous to that of 14a, starting from 25a. Purification was carried out by silica gel column chromatography (dichloromethane/methanol: 85/15). Yield: $92 \%$, m.p. $>250{ }^{\circ} \mathrm{C}\left(\mathrm{EtOAc} / \mathrm{Et}_{2} \mathrm{O}\right) .{ }^{1} \mathrm{H}$ NMR $\left(600 \mathrm{MHz}\right.$, Acetone- $\left.d_{6}\right) \delta$ 11.18-10.91 (brs, $1 \mathrm{H}, \mathrm{NH}), 8.44$ (s, $1 \mathrm{H}, \mathrm{H}-2), 8.25$ (d, $J=7.5 \mathrm{~Hz}, 2 \mathrm{H}$, phenyl H-2,6), 7.96 (s, $1 \mathrm{H}, \mathrm{H}-6), 7.37(\mathrm{t}, J=7.7 \mathrm{~Hz}, 2 \mathrm{H}$, phenyl H-3,5), $7.19(\mathrm{t}, J=7.4 \mathrm{~Hz}, 1 \mathrm{H}$, phenyl H-4), 3.96-3.90 (m, 4H, methylpiperazine $\mathrm{H}-2,6)$, 2.82-2.76 (m, 4H, methylpiperazine $\mathrm{H}-3,5), 2.47$ (s, 3H, methylpiperazine $\left.\mathrm{CH}_{3}\right) .{ }^{13} \mathrm{C}$ NMR $\left(151 \mathrm{MHz}\right.$, Acetone- $\left.d_{6}\right) \delta 151.98(\mathrm{C}-2), 150.94$ (C-4), 148.69 (C-7a), 135.32 (phenyl C-1), 129.17 (phenyl C-3,5), 127.38 (phenyl C-2,6), 126.79 (C-6), 126.54 (phenyl C-4), 116.95 (C-4a), 116.67 (C-7), 55.05 (methylpiperazine C-3,5), 46.24 (methylpiperazine C-2,6), 45.45 (methylpiperazine $\mathrm{CH}_{3}$ ). HR-MS (ESI) $\mathrm{m} / \mathrm{z}$ : Calcd for $\mathrm{C}_{17} \mathrm{H}_{20} \mathrm{~N}_{5}:[\mathrm{M}+\mathrm{H}]^{+}=294.1714$, found 294.1715. Anal. Calcd for $\mathrm{C}_{17} \mathrm{H}_{19} \mathrm{~N}_{5}: \mathrm{C}, 69.60 ; \mathrm{H}$, 6.53; N, 23.87. Found: C, 69.82; H, 6.58; N, 23.57.

7-(3-Fluorophenyl)-4-(4-methylpiperazin-1-yl)-5H-pyrrolo[3,2- $d$ ]pyrimidine (26f): This compound was synthesized using a procedure analogous to that of $\mathbf{1 4 a}$, starting from $\mathbf{2 5 b}$. Oil purification was carried out by silica gel column chromatography (dichloromethane/ methanol: $7 / 3)$. Yield: $91 \% .{ }^{1} \mathrm{H}$ NMR $\left(600 \mathrm{MHz}\right.$, Acetone- $\left.d_{6}\right) \delta 11.78-11.05$ (brs, $\left.1 \mathrm{H}, \mathrm{NH}\right)$, $8.44(\mathrm{~s}, 1 \mathrm{H}, \mathrm{H}-2), 8.22-8.16(\mathrm{~m}, 1 \mathrm{H}$, fluorophenyl H-2), $8.02(\mathrm{~s}, 1 \mathrm{H}, \mathrm{H}-6), 8.00-7.96(\mathrm{~m}, 1 \mathrm{H}$, fluorophenyl H-6), 7.42-7.34 (m, 1H, fluorophenyl H-5), 6.97-6.89 $(\mathrm{m}, 1 \mathrm{H}$, fluorophenyl $\mathrm{H}-4)$, 3.98-3.87 ( $\mathrm{m}, 4 \mathrm{H}$, methylpiperazine $\mathrm{H}-2,6)$, 2.75-2.67 ( $\mathrm{m}, 4 \mathrm{H}$, methylpiperazine $\mathrm{H}-3,5)$, 2.39 (s, 3H, methylpiperazine $\left.\mathrm{CH}_{3}\right) .{ }^{13} \mathrm{C}$ NMR $\left(151 \mathrm{MHz}\right.$, Acetone- $\left.d_{6}\right) \delta 164.86,163.26$ (fluorophenyl C-3), 152.03 (C-4), 151.14 (C-2), 148.55 (C-7a), 137.89, 137.83 (fluorophenyl C-1), 130.84, 130.78 (fluorophenyl C-5), 127.27 (C-6), 122.76 (fluorophenyl C-6), 116.85 (C-4a), 115.21 (C-7), 113.87, 113.72 (fluorophenyl C-2), 112.89, 112.75 (fluorophenyl C-4), 55.21 (methylpiperazine C-3,5), 46.38 (methylpiperazine C-2,6), 45.68 (methylpiperazine $\mathrm{CH}_{3}$ ). HR-MS (ESI) $m / z$ : Calcd for $\mathrm{C}_{17} \mathrm{H}_{19} \mathrm{FN}_{5}:[\mathrm{M}+\mathrm{H}]^{+}=312.1619$, found 312.1610. Anal. Calcd for $\mathrm{C}_{17} \mathrm{H}_{18} \mathrm{FN}_{5}$ : C, 65.58; H, 5.83; N, 22.49. Found: C, 65.66; H, 5.87; N, 22.34 .

Ethyl 4-(3-fluorophenyl)-3-(hydroxyimino)-2,4-dioxobutanoate (31b): To a solution of $30 \mathrm{~b}(1.28 \mathrm{~g}, 5.36 \mathrm{mmol})$ in glacial acetic acid $(10 \mathrm{~mL})$, a sodium nitrite solution $(3.5 \mathrm{~mL}$, $3.80 \mathrm{M}$ ) was added at $10{ }^{\circ} \mathrm{C}$, under the surface of the solution with a flow rate of 0.15 $\mathrm{mL} / \mathrm{min}$ via a controlled infusion device. The solution was stirred at $\mathrm{rt}$ for $1 \mathrm{~h}$ and then was neutralized with $25 \%$ ammonia solution and extracted with ethyl acetate; the solvent was 
dried $\left(\mathrm{Na}_{2} \mathrm{SO}_{4}\right)$ and concentrated to dryness to give pure $31 \mathbf{b}$ that corresponded practically to a sole isomer. Yield: 85\%. Amorphous solid. ${ }^{1} \mathrm{H}$ NMR (600 MHz, DMSO- $\left.d_{6}\right) \delta 12.59$ (s, $1 \mathrm{H}, \mathrm{OH})$, 7.81-7.78 (m, 1H, fluorophenyl H-6), 7.67-7.63 (m, 1H, fluorophenyl H-2), 7.58-7.55 (m, 1H, fluorophenyl H-5), 7.50-7.45 (m, 1H, fluorophenyl H-4), $4.38(\mathrm{q}, J=7.2 \mathrm{~Hz}, 2 \mathrm{H}$, ethylester H-1), $1.26\left(\mathrm{t}, J=7.2 \mathrm{~Hz}, 3 \mathrm{H}\right.$, ethylester H-2). ${ }^{13} \mathrm{C}$ NMR $\left(151 \mathrm{MHz}, \mathrm{DMSO}-d_{6}\right) \delta$ 192.03 (C-4), 166.20 (C-2), 164.47 (C-1), 162.79, 161.17 (fluorophenyl C-3), 152.09 (C-3), 133.28, 133.23 (fluorophenyl C-1), 130.84, 130.78 (fluorophenyl C-5), 125.44, 125.42 (fluorophenyl C-6), 119.94, 119.80 (fluorophenyl C-4), 115.81, 115.66 (fluorophenyl C-2), 62.76 (ethylester $\mathrm{C}-1$ ), 13.72 (ethylester C-2). HR-MS (ESI) $m / z$ : Calcd for $\mathrm{C}_{12} \mathrm{H}_{9} \mathrm{FNO}_{5}:[\mathrm{M}-\mathrm{H}]^{-}=266.0470$, found 266.0469 .

Ethyl 4-amino-3-(3-fluorophenyl)-1H-pyrazole-5-carboxylate (32b): To a solution of $31 \mathrm{~b}(1.22 \mathrm{~g}, 4.57 \mathrm{mmol})$ in absolute ethanol $(8 \mathrm{~mL})$, hydrazine hydrate $(0.5 \mathrm{~mL}, 9.14 \mathrm{mmol})$ was added dropwise, under cooling, and the resulting solution was heated at $45{ }^{\circ} \mathrm{C}$ for 2 $\mathrm{h}$. The solvent was then vacuum evaporated ethyl acetate was added to the residue and it was washed with a $3 \mathrm{~N}$ hydrochloric acid solution. The aqueous phase was neutralized with a $2 \mathrm{~N}$ sodium hydroxide solution and extracted with ethyl acetate; the organic phase was dried $\left(\mathrm{Na}_{2} \mathrm{SO}_{4}\right)$ and concentrated to dryness to give pure $\mathbf{3 2} \mathbf{b}$ as an amorphous solid. Yield: 40\%. ${ }^{1} \mathrm{H}$ NMR (600 MHz, DMSO- $\left.d_{6}\right) \delta 13.50-13.15$ (brs, $\left.1 \mathrm{H}, \mathrm{NH}\right), 7.62-7.58$ (m, 1H, fluorophenyl H-6), 7.55-7.51 (m, 1H, fluorophenyl H-2), 7.49-7.45 (m, 1H, fluorophenyl H-5), 7.14-7.09 (m, 1H, fluorophenyl H-4), 5.15-4.90 (brs, 2H, $\mathrm{NH}_{2}$ ), 4.32 (q, J = 7.2 Hz, $2 \mathrm{H}$, ethylester H-1), $1.33\left(\mathrm{t}, J=7.2 \mathrm{~Hz}, 3 \mathrm{H}\right.$, ethylester H-2). ${ }^{13} \mathrm{C}$ NMR (151 MHz, DMSO$\left.d_{6}\right) \delta$ 163.23, 161.62 (fluorophenyl C-3), 160.32 (C=O), 136.90 (C-5), 135.21 (C-3), 133.44 (fluorophenyl C-1), 130.58, 130.53 (fluorophenyl C-5), 121.49 (fluorophenyl C-6), 118.59 (C-4), 113.46, 113.32 (fluorophenyl C-4), 112.08, 111.93 (fluorophenyl C-2), 59.88 (ethylester C-1), 14.28 (ethylester C-2). HR-MS (ESI) $m / z$ : Calcd for $\mathrm{C}_{12} \mathrm{H}_{11} \mathrm{FN}_{3} \mathrm{O}_{2}$ : $[\mathrm{M}-\mathrm{H}]^{-}=248.0840$, found 248.0836 .

3-(3-Fluorophenyl)-1H-pyrazolo[4,3-d]pyrimidin-7(6H)-one (33b): To a solution of 32b (540 mg, $2.16 \mathrm{mmol})$ in butanol $(8 \mathrm{~mL})$, formamidine acetate $(500 \mathrm{mg}, 4.80 \mathrm{mmol})$ was added, and the solution was refluxed for $20 \mathrm{~h}$. Upon completion of the reaction, chloroform $(10 \mathrm{~mL})$ was added into the flask, and the precipitate was filtered and dried to give pure 33b. Yield: $61 \%$, m.p. $>250{ }^{\circ} \mathrm{C}(\mathrm{MeOH}) .{ }^{1} \mathrm{H}$ NMR $\left(600 \mathrm{MHz}, \mathrm{DMSO}-d_{6}\right) \delta$ 14.70-14.24 (brs, 1H, pyrazole NH), 12.67-12.30 (brs, 1H, pyrimidinone NH), 8.15-8.13 (m, 1H, fluorophenyl H-6), 8.11-8.07 (m, 1H, fluorophenyl H-2), 8.00 (s, 1H, H-5), 7.56-7.51 (m, 1H, fluorophenyl H-5), 7.23-7.18 (m, 1H, fluorophenyl H-4). ${ }^{13} \mathrm{C} \mathrm{NMR} \mathrm{(151} \mathrm{MHz,}$ DMSO- $d_{6}$ ) $\delta 163.15,161.54$ (fluorophenyl C-3), 153.49 (C-7), 143.65 (C-5), 140.13 (C-3), 136.50 (C-3a), 133.91 (fluorophenyl C-1), 130.75, 130.69 (fluorophenyl C-5), 129.55 (C-7a), 121.86 (fluorophenyl C-6), 114.73, 114.59 (fluorophenyl C-4), 112.43, 112.27 (fluorophenyl C-2). HR-MS (ESI) $m / z$ : Calcd for $\mathrm{C}_{11} \mathrm{H}_{6} \mathrm{FN}_{4} \mathrm{O}$ : [M-H] $]^{-}=229.0531$, found 229.0540.

7-Chloro-3-phenyl-1H-pyrazolo[4,3-d]pyrimidine (34a): Compound 33a (100 mg, 0.47 $\mathrm{mmol}$ ) was added under cooling to phosphorous oxychloride $(1.5 \mathrm{~mL})$. Then, $N, N$-diisopro pylethylamine $(122 \mu \mathrm{L}, 0.71 \mathrm{mmol})$ was added, and the mixture was refluxed for $3 \mathrm{~h}$ under argon. The bulk of the volatile material was then vacuum-evaporated, water was added into the flask and the $\mathrm{pH}$ was adjusted to 5 upon addition of a saturated sodium bicarbonate solution. The precipitate was filtered, washed with water and air-dried. The residue was purified by silica gel column chromatography (cyclohexane/ethyl acetate: $7 / 3$ ) to result in 34a (85 mg, 80\%), m.p. 220-221 ${ }^{\circ} \mathrm{C}$ (acetone). ${ }^{1} \mathrm{H}$ NMR (600 MHz, DMSO- $\left.d_{6}\right) \delta 14.80-14.55$ (brs, 1H, NH), $8.94(\mathrm{~s}, 1 \mathrm{H}, \mathrm{H}-5), 8.43(\mathrm{~d}, J=7.7 \mathrm{~Hz}, 2 \mathrm{H}$, phenyl H-2,6), 7.56 (t, $J=7.2 \mathrm{~Hz}$, $2 \mathrm{H}$, phenyl H-3,5), $7.46\left(\mathrm{t}, J=7.5 \mathrm{~Hz}, 1 \mathrm{H}\right.$, phenyl H-4). ${ }^{13} \mathrm{C}$ NMR $\left(151 \mathrm{MHz}, \mathrm{DMSO}-d_{6}\right) \delta$ 150.43 (C-5), 143.94 (C-7), 143.02 (C-3), 142.28 (C-3a), 131.03 (C-7a), 130.65 (phenyl C-1), 128.79 (phenyl C-3,5), 128.76 (phenyl C-4), 126.28 (phenyl C-2,6). HR-MS (ESI) m/z: Calcd for $\mathrm{C}_{11} \mathrm{H}_{6} \mathrm{ClN}_{4}$ : [M-H] $]^{-}=229.0286$, found 229.0301 .

7-Chloro-3-(3-fluorophenyl)-1H-pyrazolo[4,3- $d$ ]pyrimidine (34b): This compound was synthesized using a procedure analogous to that of $\mathbf{3 4 a}$, starting from $\mathbf{3 3 b}$. Purification was carried out by silica gel column chromatography (cyclohexane/ethyl acetate: 7/3). Yield: 
$80 \%$, m.p. $>250{ }^{\circ} \mathrm{C}$ (EtOAc). ${ }^{1} \mathrm{H}$ NMR $\left(600 \mathrm{MHz}, \mathrm{DMSO}-d_{6}\right) \delta 13.80-13.54$ (brs, 1H, NH), 8.96 (s, 1H, H-5), 8.30-8.27 (m, 1H, fluorophenyl H-6), 8.22-8.18 (m, 1H, fluorophenyl H-2), 7.65-7.59 (m, 1H, fluorophenyl H-5), 7.33-7.27 (m, 1H, fluorophenyl H-4). ${ }^{13} \mathrm{C}$ NMR (151 MHz, DMSO- $d_{6}$ ) $\delta 163.19,161.58$ (fluorophenyl C-3), 150.76 (C-5), 144.41 (C-7), 142.10 (C-3a), 141.52 (C-3), 133.18 (fluorophenyl C-1), 131.29 (C-7a), 131.08, 131.02 (fluorophenyl C-5), 122.27 (fluorophenyl C-6), 115.62, 115.48 (fluorophenyl C-4), 112.74, 112.58 (fluorophenyl C-2). HR-MS (ESI) $m / z$ : Calcd for $\mathrm{C}_{11} \mathrm{H}_{5} \mathrm{ClFN}_{4}$ : [M-H] $]^{-}=247.0192$, found 247.1002.

$N$,3-Diphenyl-1H-pyrazolo[4,3-d]pyrimidin-7-amine (35a): Aniline (0.1 mL, $0.85 \mathrm{mmol})$ was added into a solution of the chloride $34 \mathrm{a}(80 \mathrm{mg}, 0.34 \mathrm{mmol})$ in absolute ethanol (5 $\mathrm{mL}$ ), and the mixture was refluxed under argon for $2 \mathrm{hrs}$. Upon completion of the reaction, water $(20 \mathrm{~mL})$ was added, and the precipitate was filtered under vacuum, washed with water and air-dried. Purification was carried out by silica gel column chromatography (dichloromethane/methanol: 9/1). Yield: 61\%, m.p. 247-248 ${ }^{\circ} \mathrm{C}$ (EtOAc). ${ }^{1} \mathrm{H}$ NMR $(600$ MHz, DMSO- $d_{6}$ ) $\delta 13.20-12.90$ (brs, $1 \mathrm{H}$, pyrazole NH), 9.80-9.55 (brs, $1 \mathrm{H}$, pyrimidinamine $\mathrm{NH}$ ), 8.53 (brs, 1H, H-5), 8.44 (brs, 2H, 3-phenyl H-2,6), 7.93 (brs, 2H, N-phenyl H-2,6), 7.53 ( $\mathrm{t}, J=7.5 \mathrm{~Hz}, 2 \mathrm{H}$, 3-phenyl H-3,5), 7.45-7.38 (m, 3H, N-phenyl H-3,5, 3-phenyl H-4), $7.13\left(\mathrm{t}, J=7.4 \mathrm{~Hz}, 1 \mathrm{H}, \mathrm{N}-\right.$ phenyl H-4). ${ }^{13} \mathrm{C}$ NMR (151 MHz, DMSO-d 6 ) $\delta 151.42$ (C-5), 146.78 (C-7), 142.13 (C-3), 139.74 (C-3a), 138.91 (N-phenyl C-1), 132.16 (3-phenyl C-1), 128.89 (N-phenyl C-3,5), 128.62 (3-phenyl C-3,5), 128.08 (3-phenyl C-4), 126.15 (3-phenyl C-2,6), 123.23 (N-phenyl C-4), 122.78 (C-7a), 120.10 (N-phenyl C-2,6). HR-MS (ESI) m/z: Calcd for $\mathrm{C}_{17} \mathrm{H}_{12} \mathrm{~N}_{5}$ : [M-H] $]^{-}=286.1098$, found 286.1095. Anal. Calcd for $\mathrm{C}_{17} \mathrm{H}_{13} \mathrm{~N}_{5}$ : C, 71.06; H, 4.56; N, 24.38. Found: C, 70.88; H, 4.65; N, 24.44 .

3-(3-Fluorophenyl)- $N$-phenyl-1H-pyrazolo[4,3-d]pyrimidin-7-amine (35b): This compound was synthesized using a procedure analogous to that of $35 \mathbf{a}$ starting from $\mathbf{3 4 \mathbf { b }}$. Purification was carried out by silica gel column chromatography (dichloromethane/methanol: 98/2). Yield: 65\%, m.p. 237-238 ${ }^{\circ} \mathrm{C}$ (EtOAc). ${ }^{1} \mathrm{H}$ NMR (600 MHz, DMSO- $\left.d_{6}\right) \delta 13.35-12.95$ (brs, $1 \mathrm{H}$, pyrazole NH), 9.85-9.60 (brs, $1 \mathrm{H}$, pyrimidinamine $\mathrm{NH}), 8.55$ (s, $1 \mathrm{H}, \mathrm{H}-5), 8.30-8.26$ (m, 1H, fluorophenyl H-6), 8.25-8.21 (m, 1H, fluorophenyl H-2), 7.93 (brs, 2H, phenyl H-2,6), 7.61-7.55 (m, 1H, fluorophenyl H-5), $7.43(\mathrm{t}, J=7.4 \mathrm{~Hz}, 2 \mathrm{H}$, phenyl H-3,5), 7.26-7.21 (m, 1H, fluorophenyl H-4), $7.13\left(\mathrm{t}, J=7.4 \mathrm{~Hz}, 1 \mathrm{H}\right.$, phenyl H-4). ${ }^{13} \mathrm{C}$ NMR $\left(151 \mathrm{MHz}, \mathrm{DMSO}-d_{6}\right)$ $\delta$ 163.18, 161.57 (fluorophenyl C-3), 151.65 (C-5), 146.79 (C-7), 140.81 (C-3), 139.73 (C-3a), 138.83 (phenyl C-1), 134.43 (fluorophenyl C-1), 130.65 (fluorophenyl C-5), 128.86 (phenyl C-3,5), 123.27 (phenyl C-4), 122.87 (C-7a), 121.99 (fluorophenyl C-6), 120.13 (phenyl C-2,6), 114.75, 114.61 (fluorophenyl C-4), 112.57, 112.42 (fluorophenyl C-2). HR-MS (ESI) $\mathrm{m} / \mathrm{z}$ : Calcd for $\mathrm{C}_{17} \mathrm{H}_{11} \mathrm{FN}_{5}:[\mathrm{M}-\mathrm{H}]^{-}=304.1003$, found 304.0998. Anal. Calcd for $\mathrm{C}_{17} \mathrm{H}_{12} \mathrm{FN}_{5}: \mathrm{C}$, 66.88; H, 3.96; N, 22.94. Found: C, 66.61; H, 4.02; N, 23.09.

3-Phenyl- $N$-(3,4,5-trimethoxyphenyl)-1H-pyrazolo[4,3- $d$ ]pyrimidin-7-amine (35c): This compound was synthesized using a procedure analogous to that of 35a starting from 34a. Purification was carried out by silica gel column chromatography (dichloromethane/ methanol: 95/5). Yield: 68\%, m.p. > $250{ }^{\circ} \mathrm{C}$ (EtOAc). ${ }^{1} \mathrm{H}$ NMR $\left(600 \mathrm{MHz}, \mathrm{DMSO}-d_{6}\right) \delta 13.10$ 12.80 (brs, $1 \mathrm{H}$, pyrazole NH), 9.70-9.50 (brs, $1 \mathrm{H}$, pyrimidinamine $\mathrm{NH}), 8.53$ (s, 1H, H-5), 8.43 (brs, 2H, phenyl H-2,6), $7.53(\mathrm{t}, J=7.1 \mathrm{~Hz}, 2 \mathrm{H}$, phenyl H-3,5), $7.40(\mathrm{t}, J=7.4 \mathrm{~Hz}, 1 \mathrm{H}$, phenyl $\mathrm{H}-4)$, 7.35-7.20 (brs, 2H, trimethoxyphenyl $\mathrm{H}-2,6), 3.83$ (s, $6 \mathrm{H}$, trimethoxyphenyl $\mathrm{CH}_{3} \mathrm{O}-$ 3,5), 3.68 (s, 3H, trimethoxyphenyl $\left.\mathrm{CH}_{3} \mathrm{O}-4\right) .{ }^{13} \mathrm{C}$ NMR $\left(151 \mathrm{MHz}, \mathrm{DMSO}-d_{6}\right) \delta 152.88$ (trimethoxyphenyl C-3,5), 151.44 (C-5), 146.79 (C-7), 142.13 (C-3), 139.67 (C-3a), 134.96 (C-7a), 133.70 (trimethoxyphenyl C-4), 132.18 (phenyl C-1), 128.62 (phenyl C-3,5), 128.08 (phenyl C-4), 126.18 (phenyl C-2,6), 122.75 (trimethoxyphenyl C-1), 98.25 (trimethoxyphenyl $\mathrm{C}-2,6), 60.13$ (trimethoxyphenyl $\mathrm{CH}_{3} \mathrm{O}-4$ ), 55.88 (trimethoxyphenyl $\left.\mathrm{CH}_{3} \mathrm{O}-3,5\right)$. HR-MS (ESI) $m / z$ : Calcd for $\mathrm{C}_{20} \mathrm{H}_{18} \mathrm{~N}_{5} \mathrm{O}_{3}$ : [M-H] $]^{-}=376.1415$, found 376.1408. Anal. Calcd for $\mathrm{C}_{20} \mathrm{H}_{19} \mathrm{~N}_{5} \mathrm{O}_{3}$ : C, 63.65; H, 5.07; N, 18.56. Found: C, 63.90; H, 5.01; N, 18.69.

3-(3-Fluorophenyl)- $N$-(3,4,5-trimethoxyphenyl)- $1 H$-pyrazolo[4,3-d]pyrimidin-7-amine (35d): This compound was synthesized using a procedure analogous to that of 35a starting from 34b. Purification was carried out by silica gel column chromatography (dichlorometha ne/methanol: 98/2). Yield: 57\%, m.p. > $250{ }^{\circ} \mathrm{C}$ (EtOAc). ${ }^{1} \mathrm{H}$ NMR (600 MHz, DMSO- $\left.d_{6}\right)$ 
$\delta$ 13.12-12.96 (brs, $1 \mathrm{H}$, pyrazole $\mathrm{NH}), 9.66-9.54$ (brs, $1 \mathrm{H}$, pyrimidinamine $\mathrm{NH}), 8.54(\mathrm{~s}$, $1 \mathrm{H}, \mathrm{H}-5), 8.30-8.19(\mathrm{~m}, 2 \mathrm{H}$, fluorophenyl H-6, fluorophenyl $\mathrm{H}-2), 7.60-7.53(\mathrm{~m}, 1 \mathrm{H}$, fluorophenyl H-5), 7.30-7.19 $(\mathrm{m}, 3 \mathrm{H}$, trimethoxyphenyl $\mathrm{H}-2,6$, fluorophenyl $\mathrm{H}-4), 3.83(\mathrm{~s}, 6 \mathrm{H}$, trimethoxyphenyl $\left.\mathrm{CH}_{3} \mathrm{O}-3,5\right), 3.68\left(\mathrm{~s}, 3 \mathrm{H}\right.$, trimethoxyphenyl $\left.\mathrm{CH}_{3} \mathrm{O}-4\right) .{ }^{13} \mathrm{C} \mathrm{NMR}(151 \mathrm{MHz}$, DMSO- $d_{6}$ ) $\delta 163.19,161.58$ (fluorophenyl C-3), 152.85 (C-5), 151.73 (trimethoxyphenyl C3,5), 146.79 (C-7), 140.82 (C-3), 139.65 (C-3a), 134.85 (C-7a), 133.78 (trimethoxyphenyl C-4), 130.76 (trimethoxyphenyl C-1, fluorophenyl C-5), 122.80 (fluorophenyl C-1), 121.97 (fluorophenyl C-6), 114.81, 114.67 (fluorophenyl C-4), 112.52, 112.36 (fluorophenyl C-2), 98.36 (trimethoxyphenyl C-2,6), 60.12 (trimethoxyphenyl $\mathrm{CH}_{3} \mathrm{O}-4$ ), 55.88 (trimethoxyphenyl $\mathrm{CH}_{3} \mathrm{O}-3,5$ ). HR-MS (ESI) $\mathrm{m} / z$ : Calcd for $\mathrm{C}_{20} \mathrm{H}_{19} \mathrm{FN}_{5} \mathrm{O}_{3}$ : $[\mathrm{M}+\mathrm{H}]^{+}=396.1467$, found 396.1455. Anal. Calcd for $\mathrm{C}_{20} \mathrm{H}_{18} \mathrm{FN}_{5} \mathrm{O}_{3}: \mathrm{C}, 60.75 ; \mathrm{H}, 4.59 ; \mathrm{N}, 17.71$. Found: $\mathrm{C}, 60.94 ; \mathrm{H}, 4.46$; $\mathrm{N}, 17.59$.

7-(4-Methylpiperazin-1-yl)-3-phenyl-1H-pyrazolo[4,3- $d$ ]pyrimidine (35e): This compound was synthesized using a procedure analogous to that of 35a starting from 34a. Upon completion of the reaction, the solvent was evaporated and water $(20 \mathrm{~mL})$ was added into the flask, followed by extraction with ethyl acetate $(3 \times 20 \mathrm{~mL})$. The combined organic layers were dried $\left(\mathrm{Na}_{2} \mathrm{SO}_{4}\right)$ and evaporated to dryness. Purification was carried out by silica gel column chromatography (dichloromethane/methanol: 9/1). Yield: 80\%, m.p. 184-185 ${ }^{\circ} \mathrm{C}$ (EtOAc). ${ }^{1} \mathrm{H}$ NMR $\left(600 \mathrm{MHz}\right.$, DMSO- $\left.d_{6}\right) \delta 8.35(\mathrm{~d}, J=7.9 \mathrm{~Hz}, 2 \mathrm{H}$, phenyl H-2,6), $8.30(\mathrm{~s}, 1 \mathrm{H}, \mathrm{H}-5), 7.52(\mathrm{t}, J=7.2 \mathrm{~Hz}, 2 \mathrm{H}$, phenyl H-3,5), $7.38(\mathrm{t}, J=7.5 \mathrm{~Hz}, 1 \mathrm{H}$, phenyl H-4), 4.25-4.10 (brs, 4H, methylpiperazine $\mathrm{H}-2,6)$, 2.49-2.46 (m, 4H, methylpiperazine $\mathrm{H}-3,5), 2.24$ (s, 3H, methylpiperazine $\left.\mathrm{CH}_{3}\right) .{ }^{13} \mathrm{C}$ NMR (151 MHz, DMSO- $\left.d_{6}\right) \delta 152.80$ (C-7), 151.80 (C-5), 138.33 (C-3a), 134.43 (C-3), 129.56 (C-7a, phenyl C-1), 128.76 (phenyl C-3,5), 128.01 (phenyl $\mathrm{C}-4), 125.73$ (phenyl C-2,6), 54.53 (methylpiperazine $\mathrm{C}-3,5), 45.59$ (methylpiperazine $\mathrm{CH}_{3}$ ), 44.89 (methylpiperazine C-2,6). HR-MS (ESI) $m / z$ : Calcd for $\mathrm{C}_{16} \mathrm{H}_{17} \mathrm{~N}_{6}:[\mathrm{M}-\mathrm{H}]^{-}=293.1520$, found 293.1499. Anal. Calcd for $\mathrm{C}_{16} \mathrm{H}_{18} \mathrm{~N}_{6}$ : $\mathrm{C}, 65.29 ; \mathrm{H}, 6.16 ; \mathrm{N}, 28.55$. Found: $\mathrm{C}, 65.44 ; \mathrm{H}$, $6.08 ; \mathrm{N}, 28.31$.

3-(3-Fluorophenyl)-7-(4-methylpiperazin-1-yl)-1H-pyrazolo[4,3- $d$ ]pyrimidine (35f): This compound was synthesized using a procedure analogous to that of 35 a starting from $34 \mathbf{b}$. The work-up procedure was similar to that of the derivative 35e. Purification was carried out by silica gel column chromatography (dichloromethane/methanol: 93/7). Yield: $80 \%$, m.p. $212-213^{\circ} \mathrm{C}$ (EtOAc). ${ }^{1} \mathrm{H}$ NMR $\left(600 \mathrm{MHz}\right.$, DMSO- $\left.d_{6}\right) \delta 8.32$ (s, $\left.1 \mathrm{H}, \mathrm{H}-5\right)$, 8.22-8.19 (m, 2H, fluorophenyl H-2, fluorophenyl H-6), 7.58-7.53 $(\mathrm{m}, 1 \mathrm{H}$, fluorophenyl $\mathrm{H}-5)$, 7.22-7.18 (m, $1 \mathrm{H}$, fluorophenyl $\mathrm{H}-4)$, 4.18-4.12 (brs, 4H, methylpiperazine $\mathrm{H}-2,6)$, 2.48-2.45 (m, 4H, methylpiperazine $\mathrm{H}-3,5), 2.23\left(\mathrm{~s}, 3 \mathrm{H}\right.$, methylpiperazine $\left.\mathrm{CH}_{3}\right) .{ }^{13} \mathrm{C}$ NMR $\left(151 \mathrm{MHz}, \mathrm{DMSO}-d_{6}\right) \delta 163.17,161.56$ (fluorophenyl C-3), 152.52 (C-7), 152.03 (C-5), 138.82 (C-3a), 133.93 (C-3), 132.01, 131.96 (fluorophenyl C-5), 130.84, 130.78 (fluorophenyl C-1), 129.23 (C-7a), 121.58 (fluorophenyl C-6), 114.64, 114.50 (fluorophenyl C-4), 112.21, 112.06 (fluorophenyl C-2), 54.48 (methylpiperazine C-3,5), 45.55 (methylpiperazine $\mathrm{CH}_{3}$ ), 44.92 (methylpiperazine C-2,6). HR-MS (ESI) $m / z$ : Calcd for $\mathrm{C}_{16} \mathrm{H}_{16} \mathrm{FN}_{6}$ : $[\mathrm{M}-\mathrm{H}]^{-}=311.1425$, found 311.1455. Anal. Calcd for $\mathrm{C}_{16} \mathrm{H}_{17} \mathrm{FN}_{6}$ : $\mathrm{C}, 61.53 ; \mathrm{H}, 5.49 ; \mathrm{N}, 26.91$. Found: $\mathrm{C}, 61.61 ; \mathrm{H}$, $5.36 ; \mathrm{N}, 27.00$.

\subsection{Cell Viability Assays}

The human HCT116 colon cancer cell line and PC-3 prostate cancer cell line were obtained from the American Type Cell Culture (ATCC, Bethesda, Md). Both cell lines were grown in $75 \mathrm{~cm}^{2}$ culture flasks at $37{ }^{\circ} \mathrm{C}$ in $5 \% \mathrm{CO}_{2}$ using Roswell Park Memorial Institute 1640 medium (RPMI 1640, Gibco) and Dulbecco's modified Eagle's medium F/12 (DMEM/F-12, Gibco), respectively, containing 10\% fetal bovine serum (FBS, Gibco). To test the inhibitory activities of compounds using a cell-based assay, MTT assay was performed for cell viability, as described previously [42]. Briefly, HCT116 cells were plated at a density of 1500 per well, while PC-3 cells were plated at a density of 750 per well in a 96-well plate. After $24 \mathrm{~h}$, cells were treated with the indicated compounds in a dose-dependent manner for $72 \mathrm{~h}$ and $96 \mathrm{~h}$ (all tested compounds provided clear solutions). MTT [3-(4,5- 
dimethylthiazol-2-yl)-2,5-diphenyltetrazolium bromide] (Sigma M-5655) was added at a final concentration of $0.5 \mathrm{mg} / \mathrm{mL}$ directly to each well for $4 \mathrm{~h}$ at $37^{\circ} \mathrm{C}$. The medium was aspirated, and the blue MTT formazan precipitate was dissolved in dimethyl sulfoxide (DMSO). Absorbance was determined in a Powerwave microplate spectrophotometer (Biotek Instruments, Inc.) at $540 \mathrm{~nm}$. Viable cell numbers were determined by tetrazolium conversion to its formazan dye. The $\mathrm{IC}_{50}$ was calculated by Microsoft Excel equation and confirmed by GraphPad Prism (7.0). Each experiment was performed in triplicate and mean values $\pm \mathrm{SD}$ are reported.

\subsection{DNA Staining and Flow Cytometric Analysis}

Exponentially growing PC-3 cells were treated with the $\mathrm{IC}_{50}$ values of the compounds $\mathbf{1 4 b}, \mathbf{2 6 b}$ and $\mathbf{3 5 b}$ or the corresponding DMSO concentration (vehicle) for $72 \mathrm{~h}$. For cell cycle analysis, cell culture supernatants and attached cells were collected, centrifuged, washed in PBS, fixed in 50\% ethanol and stained with an RNase-containing propidium iodide solution $(50 \mu \mathrm{g} / \mathrm{mL})$ (all reagents from Sigma). For cell apoptosis assay, cells were harvested and stained with Annexin V binding buffer, Annexin V-FITC and 7-AAD (Annexin V-FITC Apoptosis Detection Kit, BD Systems). DNA content was analyzed on a BD Accuri C6 Flow Cytometer using the BD CSampler software (BD Biosciences, USA). Non-apoptotic events were used to calculate the percentage of cells distributed in each phase. A $p$ value $<0.05$ was considered to be statistically significant (Student's $t$-test).

\section{Conclusions}

In conclusion, we have designed and synthesized a number of new substituted pyrrolo[2,3-c]pyridines, pyrrolo[3,2- $d]$ pyrimidines and pyrazolo[4,3-d]pyrimidines and have evaluated their antiproliferative activity against two cancer cell lines. We preserved an analogous substitution pattern around the fused ring-system of all three scaffolds in order to assist the extraction of SARs. We have identified a number of derivatives with potent cytotoxic properties-more precisely, the pyrrolopyridines $\mathbf{1 4 a - f}$, the pyrrolopyrimidines $\mathbf{2 6 a}, \mathbf{b}$ and the pyrazolopyrimidines $35 \mathbf{a}, \mathbf{b}$, all of which possess $\mathrm{IC}_{50}$ values in the $\mathrm{nM}$ to low $\mu \mathrm{M}$ level against both cell lines tested. We assume that in all cases the simultaneous presence of a 3-fluorophenyl moiety and a phenylamino substituent at analogous positions resulted in highly active compounds which induce apoptosis in PC-3 cells and, importantly, are devoid of toxicity against normal cells. These interesting observations have triggered the continuation of research efforts in our laboratory for the investigation of the exact mechanism of action of this promising class of compounds.

Supplementary Materials: The following supporting information can be downloaded online: Figures S1-S55: ${ }^{1} \mathrm{H}-\mathrm{NMR}$ and ${ }^{13} \mathrm{C}$ NMR spectra of new compounds, HRMS spectra of active compounds; Table S1: Calculated physicochemical characteristics of the tested compounds using SwissADME.

Author Contributions: Conceptualization, P.M. and N.P.; methodology, S.D., E.-S.G., A.P., N.L., K.G. and R.T.; validation, N.L., R.T., P.M. and N.P.; formal analysis, S.D., E.-S.G., A.P. and K.G.; data curation, N.L., P.M., N.P. and R.T.; writing-original draft preparation, N.L., P.M., N.P. and R.T.; writing-review and editing, N.L., P.M., N.P. and R.T.; supervision, P.M. All authors have read and agreed to the published version of the manuscript.

Funding: This research received no external funding.

Acknowledgments: The authors are grateful to Emmanuel Mikros who performed the physicochemical calculations and participated in useful discussion concerning the evaluation of the results of this work.

Conflicts of Interest: The authors declare no conflict of interest.

Sample Availability: Samples of the compounds 14a-f, 26a-f, 35a-f are available from the authors.

\section{References}

1. Motati, D.R.; Amaradhi, R.; Ganesh, T. Azaindole therapeutic agents. Bioorg. Med. Chem. 2020, 28, 115830. [CrossRef] [PubMed] 
2. Irie, T.; Sawa, M. 7-Azaindole: A Versatile Scaffold for Developing Kinase Inhibitors. Chem. Pharm. Bull. 2018, 66, 29-36. [CrossRef]

3. Han, Y.; Dong, W.; Guo, Q.; Li, X.; Huang, L. The importance of indole and azaindole scaffold in the development of antitumor agents. Eur. J. Med. Chem. 2020, 203, 112506. [CrossRef] [PubMed]

4. Maemoto, T.; Finlayson, K.; Olverman, H.J.; Akahane, A.; Horton, R.W.; Butcher, S.P. Species differences in brain adenosine $\mathrm{A}_{1}$ receptor pharmacology revealed by use of xanthine and pyrazolopyridine based antagonists. Br. J. Pharmacol. 1997, 122, 1202-1208. [CrossRef] [PubMed]

5. Hamajima, T.; Takahasi, F.; Kato, K.; Sugano, Y.; Yamaki, S.; Moritomo, A.; Kubo, S.; Nakamura, K.; Yamagami, K.; Hamakawa, N.; et al. Optimization and in vivo evaluation of pyrazolopyridines as a potent and selective PI3K $\delta$ inhibitor. Bioorg. Med. Chem. 2018, 26, 3917-3924. [CrossRef]

6. Eissa, I.H.; El-Naggar, A.M.; El-Hashash, M.A. Design, synthesis, molecular modeling and biological evaluation of novel 1H-pyrazolo[3,4- $b$ ]pyridine derivatives as potential anticancer agents. Bioorg. Chem. 2016, 67, 43-56. [CrossRef]

7. Michailidou, M.; Giannouli, V.; Kotsikoris, V.; Papadodima, O.; Kontogianni, G.; Kostakis, I.K.; Lougiakis, N.; Chatziioannou, A.; Kolisis, F.N.; Marakos, P.; et al. Novel pyrazolopyridine derivatives as potential angiogenesis inhibitors: Synthesis, biological evaluation and transcriptome-based mechanistic analysis. Eur. J. Med. Chem. 2016, 121, 143-157. [CrossRef]

8. Pathania, S.; Rawal, R.K. Pyrrolopyrimidines: An update on recent advancements in their medicinal attributes. Eur. J. Med. Chem. 2018, 157, 503-526. [CrossRef]

9. El-Gohary, N.S.; Shaaban, M.I. New pyrazolopyridine analogs: Synthesis, antimicrobial, antiquorum-sensing and antitumor screening. Eur. J. Med. Chem. 2018, 152, 126-136. [CrossRef] [PubMed]

10. Salem, M.S.; Ali, M.A.M. Novel Pyrazolo[3,4- $b$ ]pyridine Derivatives: Synthesis, Characterization, Antimicrobial and Antiproliferative Profile. Biol. Pharm. Bull. 2016, 39, 473-483. [CrossRef]

11. McGowan, D.C.; Balemans, W.; Embrechts, W.; Motte, M.; Keown, J.R.; Buyck, C.; Corbera, J.; Funes, M.; Moreno, L.; Cooymans, L.; et al. Design, Synthesis, and Biological Evaluation of Novel Indoles Targeting the Influenza PB2 Cap Binding Region. J. Med. Chem. 2019, 62, 9680-9690. [CrossRef] [PubMed]

12. Patnaik, S.; Basu, D.; Southall, N.; Dehdashti, S.; Wan, K.K.; Zheng, W.; Ferrer, M.; Taylor, M.; Engel, D.A.; Marugan, J.J. Identification, design and synthesis of novel pyrazolopyridine influenza virus nonstructural protein 1 antagonists. Bioorg. Med. Chem. Lett. 2019, 29, 1113-1119. [CrossRef] [PubMed]

13. Pinheiro, L.C.S.; Feitosa, L.M.; Gandi, M.O.; Silveira, F.F.; Boechat, N. The Development of Novel Compounds Against Malaria: Quinolines, Triazolpyridines, Pyrazolopyridines and Pyrazolopyrimidines. Molecules 2019, 24, 4095. [CrossRef] [PubMed]

14. Anand, D.; Yadav, P.K.; Patel, O.P.S.; Parmar, N.; Maurya, R.K.; Vishwakarma, P.; Raju, K.S.R.; Taneja, I.; Wahajuddin, M.; Kar, S.; et al. Antileishmanial Activity of Pyrazolopyridine Derivatives and Their Potential as an Adjunct Therapy with Miltefosine. $J$. Med. Chem. 2017, 60, 1041-1059. [CrossRef]

15. Pfefferkorn, J.A.; Tu, M.; Filipski, K.J.; Guzman-Perez, A.; Bian, J.; Aspnes, G.E.; Sammons, M.F.; Song, W.; Li, J.C.; Jones, C.S.; et al. The design and synthesis of indazole and pyrazolopyridine based glucokinase activators for the treatment of type 2 diabetes mellitus. Bioorg. Med. Chem. Lett. 2012, 22, 7100-7105. [CrossRef] [PubMed]

16. da Silva Lima, C.H.; de Araujo Vanelis Soares, J.C.; de Sousa Ribeiro, J.L.; Muri, E.M.F.; de Albuquerque, S.; Dias, L.R.S. AntiTrypanosoma cruzi Activity and Molecular Docking Studies of $1 H$-pyrazolo[ 3, 4-b]pyridine Derivatives. Lett. Drug Des. Discov. 2020, 17, 184-191. [CrossRef]

17. Jouha, J.; Loubidi, M.; Bouali, J.; Hamri, S.; Hafid, A.; Suzenet, F.; Guillaumet, G.; Dagcı, T.; Khouili, M.; Aydın, F.; et al. Synthesis of new heterocyclic compounds based on pyrazolopyridine scaffold and evaluation of their neuroprotective potential in $\mathrm{MPP}^{+}$-induced neurodegeneration. Eur. J. Med. Chem. 2017, 129, 41-52. [CrossRef]

18. Pennington, L.D.; Moustakas, D.T. The necessary nitrogen atom: A versatile high-impact design element for multiparameter optimization. J. Med. Chem. 2017, 60, 3552-3579. [CrossRef]

19. Bedard, P.L.; Hyman, D.M.; Davids, M.S.; Siu, L.L. Small molecules, big impact: 20 years of targeted therapy in oncology. Lancet 2020, 395, 1078-1088. [CrossRef]

20. Agianian, B.; Gavathiotis, E. Current Insights of BRAF Inhibitors in Cancer. J. Med. Chem. 2018, 61, 5775-5793. [CrossRef] [PubMed]

21. Bhullar, K.S.; Lagarón, N.O.; McGowan, E.M.; Parmar, I.; Jha, A.; Hubbard, B.P.; Vasantha Rupasinghe, H.P. Kinase-targeted cancer therapies: Progress, challenges and future directions. Mol. Cancer 2018, 17, 48. [CrossRef] [PubMed]

22. Giannouli, V.; Lougiakis, N.; Kostakis, I.K.; Pouli, N.; Marakos, P.; Skaltsounis, A.-L.; Nam, S.; Jove, R.; Horne, D.; Tenta, R.; et al. The discovery of new cytotoxic pyrazolopyridine derivatives. Bioorg. Med. Chem. Lett. 2016, 26, 5229-5233. [CrossRef]

23. Gavriil, E.-S.; Lougiakis, N.; Pouli, N.; Marakos, P.; Skaltsounis, A.-L.; Nam, S.; Jove, R.; Horne, D.; Gioti, K.; Pratsinis, H.; et al. Synthesis and antiproliferative activity of new pyrazolo[3,4-c]pyridines. Med. Chem. 2017, 13, 365-374. [CrossRef]

24. Gavriil, E.-S.; Doukatas, A.; Karampelas, T.; Myrianthopoulos, V.; Dimitrakis, S.; Mikros, E.; Marakos, P.; Tamvakopoulos, C.; Pouli, N. Design, Synthesis and Biological Evaluation of Novel Substituted Purine Isosters as EGFR kinase inhibitors, with promising pharmacokinetic profile and in vivo efficacy. Eur. J. Med. Chem. 2019, 176, 393-409. [CrossRef]

25. Papastathopoulos, A.; Lougiakis, N.; Kostakis, I.K.; Marakos, P.; Pouli, N.; Pratsinis, H.; Kletsas, D. New bioactive 5arylcarboximidamidopyrazolo[3,4-c]pyridines: Synthesis, cytotoxic activity, mechanistic investigation and structure-activity relationships. Eur. J. Med. Chem. 2021, 218, 113387. [CrossRef] 
26. Brown, E.V. Syntheses and Decarboxylation of the Isomeric Nitropyridinecarboxylic Acids. J. Am. Chem. Soc. 1954, 76, 3167-3168. [CrossRef]

27. Linz, S.; Müller, J.; Hübner, H.; Gmeiner, P.; Troschütz, R. Design, synthesis and dopamine D4 receptor binding activities of new N-heteroaromatic 5/6-ring Mannich bases. Bioorg. Med. Chem. 2009, 17, 4448-4458. [CrossRef]

28. Theoclitou, M.-E.; Aquila, B.; Block, M.H.; Brassil, P.J.; Castriotta, L.; Code, E.; Collins, M.P.; Davies, A.M.; Deegan, T.; Ezhuthachan, J.; et al. Discovery of (+)-N-(3-Aminopropyl)-N-[1-(5-benzyl-3-methyl-4-oxo-[1,2]thiazolo[5,4-d]pyrimidin-6-yl)-2-methylpropyl]4-methylbenzamide (AZD4877), a Kinesin Spindle Protein Inhibitor and Potential Anticancer Agent. J. Med. Chem. 2011, 54, 6734-6750. [CrossRef] [PubMed]

29. Furneaux, R.H.; Tyler, P.C. Improved Syntheses of 3H,5H-Pyrrolo[3,2-d]pyrimidines. J. Org. Chem. 1999, 64, 8411-8412. [CrossRef]

30. Haraguchi, K.; Horii, C.; Yoshimura, Y.; Ariga, F.; Tadokoro, A.; Tanaka, H. An Access to the $\beta$-Anomer of $4^{\prime}-$ Thio-Cribonucleosides: Hydroboration of 1-C-Aryl- or 1-C-Heteroaryl-4-thiofuranoid Glycals and Its Regiochemical Outcome. J. Org. Chem. 2011, 76, 8658-8669. [CrossRef]

31. Kaiser, M.M.; Baszczyňski, O.; Hocková, D.; Poštová-Slavětínská, L.; Dračínský, M.; Keough, D.T.; Guddat, L.W.; Janeba, Z. Acyclic Nucleoside Phosphonates Containing 9-Deazahypoxanthine and a Five-Membered Heterocycle as Selective Inhibitors of Plasmodial 6-Oxopurine Phosphoribosyltransferases. Chem. Med. Chem. 2017, 12, 1133-1141. [CrossRef]

32. Garel, L.; Saint-Jalmes, L. One-pot fluoro-de-diazoniation of anilines in organic medium. Tet. Lett. 2006, 47, 5705-5708. [CrossRef]

33. Tabrizi, M.A.; Baraldi, P.G.; Baraldi, S.; Ruggiero, E.; De Stefano, L.; Rizzolio, F.; Di Cesare Mannelli, L.; Ghelardini, C.; Chicca, A.; Lapillo, M.; et al. Discovery of 1,5-Diphenylpyrazole-3-Carboxamide Derivatives as Potent, Reversible, and Selective Monoacylglycerol Lipase (MAGL) Inhibitors. J. Med. Chem. 2018, 61, 1340-1354. [CrossRef]

34. Hoveyda, H.R.; Roy, M.-O.; Blanc, S.; Noël, S.; Salvino, J.M.; Ator, M.A.; Fraser, G. Discovery of 3-aryl-5-acylpiperazinyl-pyrazoles as antagonists to the NK3 receptor. Bioorg. Med. Chem. Lett. 2011, 21, 1991-1996. [CrossRef] [PubMed]

35. Yuan, J.; Gulianello, M.; De Lombaert, S.; Brodbeck, R.; Kieltyka, A.; Hodgetts, K.J. 3-Aryl pyrazolo[4,3-d]pyrimidine derivatives: Nonpeptide CRF-1 antagonists. Bioorg. Med. Chem. Lett. 2002, 12, 2133-2136. [CrossRef]

36. Majid, T.; Hopkins, C.R.; Pedgrift, B.; Collar, N. Convenient synthesis of 4-amino-3,5-disubstituted pyrazoles in one step from the corresponding diketo oximes. Tet. Lett. 2004, 45, 2137-2139. [CrossRef]

37. Geffken, D.; Soliman, R.; Soliman, F.S.G.; Abdel-Khalek, M.M.; Issa, D.A.E. Synthesis of new series of pyrazolo[4,3-d]pyrimidin-7ones and pyrido[2,3-d]pyrimidin-4-ones for their bacterial and cyclin-dependent kinases (CDKs) inhibitory activities. Med. Chem. Res. 2011, 20, 408-420. [CrossRef]

38. Daina, A.; Michielin, O.; Zoete, V. SwissADME: A free web tool to evaluate pharmacokinetics, drug-likeness and medicinal chemistry friendliness of small molecules. Sci. Rep. 2017, 7, 42717. [CrossRef]

39. SIMCA, version 14.1; Umetrics: Malmö, Sweden, 2015.

40. Gioti, K.; Papachristodoulou, A.; Benaki, D.; Beloukas, A.; Vontzalidou, A.; Aligiannis, N.; Skaltsounis, A.L.; Mikros, E.; Tenta, R. Glycyrrhiza glabra-Enhanced Extract and Adriamycin Antiproliferative Effect on PC-3 Prostate Cancer Cells. Nutr. Cancer 2020, 72, 320-332. [CrossRef]

41. Hanney, B.; Manley, P.; Rudd, M.T.; Sanders, J.M.; Stachel, S.J.; Henze, D. Merck Sharp \& Dohme Corp. WO2013/9582 (A1), 17 January 2013.

42. Vistica, D.T.; Skehan, P.; Scudiero, D.; Monks, A.; Pittman, A.; Boyd, M.R. Tetrazolium-based assays for cellular viability: A critical examination of selected parameters affecting formazan production. Cancer Res. 1991, 51, 2515-2520. 